Illinois State University

ISU ReD: Research and eData

Theses and Dissertations

$12-21-2020$

\title{
A Molecular Toolset For The In Vivo Detection Of A Sulfolobus Islandicus Leucyl Trna Synthetase Paralog
}

Nicholas Michael Bretz

Illinois State University, nbretz@ilstu.edu

Follow this and additional works at: https://ir.library.illinoisstate.edu/etd

Part of the Biochemistry Commons, Cell Biology Commons, and the Genetics Commons

\section{Recommended Citation}

Bretz, Nicholas Michael, "A Molecular Toolset For The In Vivo Detection Of A Sulfolobus Islandicus Leucyl Trna Synthetase Paralog" (2020). Theses and Dissertations. 1354.

https://ir.library.illinoisstate.edu/etd/1354

This Thesis is brought to you for free and open access by ISU ReD: Research and eData. It has been accepted for inclusion in Theses and Dissertations by an authorized administrator of ISU ReD: Research and eData. For more information, please contact ISUReD@ilstu.edu. 


\section{A MOLECULAR TOOLSET FOR THE IN VIVO DETECTION OF A SULFOLOBUS \\ ISLANDICUS LEUCYL TRNA SYNTHETASE PARALOG}

\section{NICHOLAS MICHAEL BRETZ}

100 Pages

An ancient and ubiquitous set of enzymes known as the aminoacyl-tRNA synthetases are required for the viability of all organisms. The aminoacyl-tRNA synthetases catalyze the attachment of amino acids onto tRNA molecules. The aminoacylated, or charged, tRNA is then transported and utilized at the ribosome for the synthesis of proteins. The genome of the hyperthermophilic microorganism Sulfolobus islandicus (S. islandicus) harbors a unique leucyltRNA synthetase paralog, LeuRS-I, of unknown function. While its duplicate, LeuRS-F, carries out all the classical functions of LeuRS-family enzymes, LeuRS-I fails to charge leucine onto tRNA ${ }^{\text {Leu }}$, despite its ability to activate this amino acid and bind this tRNA substrate. To begin to understand the molecular function of this unusual LeuRS within S. islandicus, new approaches of inquiry are needed. A variety of biotechnological methods were developed in an attempt to piece together the activity of this paralogous synthetase and understand the role it plays within $S$. islandicus.

KEYWORDS: Archaea, Sulfolobus, aminoacyl-tRNA synthetase (aaRS), LeuRS, paralogs, polyclonal antibody, tRNA extraction, EMSA, microscale thermophoresis 
A MOLECULAR TOOLSET FOR THE IN VIVO DETECTION OF A SULFOLOBUS

ISLANDICUS LEUCYL TRNA SYNTHETASE PARALOG

NICHOLAS MICHAEL BRETZ

\author{
A Thesis Submitted in Partial \\ Fulfillment of the Requirements \\ for the Degree of \\ MASTER OF SCIENCE \\ School of Biological Sciences \\ ILLINOIS STATE UNIVERSITY
}

2021 
(C) 2021 Nicholas Michael Bretz 
A MOLECULAR TOOLSET FOR THE IN VIVO DETECTION OF A SULFOLOBUS

ISLANDICUS LEUCYL TRNA SYNTHETASE PARALOG

NICHOLAS MICHAEL BRETZ

COMMITTEE MEMBERS:

Christopher S. Weitzel, Chair

Jan Dahl

Nathan Mortimer 


\section{ACKNOWLEDGMENTS}

I thank Dr. Christopher S. Weitzel for taking me on as an undergraduate researcher and teaching me what it means to be a good scientist, both philosophically and practically. Without his persistent guidance, I would not be here today writing this paragraph. I would also like to thank my lab members, many of whom have bolstered my resolve when I was weak and at a wit's end during times of extreme difficulty in the lab - you are all dearly appreciated friends. I would also like to thank my parents. Without them, I would not be the man I am today, nor would I have been afforded the opportunity to further my education following high school. Finally, I would like to give a major thanks to my committee. Nate and Jan have provided generous feedback regarding my research, and for that I am greatly appreciative.

N.M.B. 


\section{CONTENTS}

Page

ACKNOWLEDGMENTS

CONTENTS

ii

FIGURES

CHAPTER I: INTRODUCTION 1

The aminoacyl-tRNA synthetases - an overview 1

Sulfolobus islandicus, a model hyperthermophilic organism 2

Duplication of aaRSs and the act of mistranslation 3

Duplication of LeuRS in Sulfolobus islandicus - potential ramifications $\quad 8$

$\begin{array}{ll}\text { Summary of research objectives } & 11\end{array}$

CHAPTER II: EXPERIMENTAL METHODS 13

tRNA Extractions $\quad 13$

Method I: Small-scale extraction of total S. islandicus tRNA

Method II: Large-scale extraction of total S. islandicus tRNA 16

Method III: Agarose bleach gel electrophoresis 18

Method IV: Small RNA enrichment 18

Electrophoretic mobility shift assays and microscale thermophoresis 
Method I: Electrophoretic mobility shift assays using radiolabeled tRNA ${ }^{\text {Leu }}$-UAG

and SYBR Gold

Method II: Microscale thermophoresis (MST)

Phylogenetic analyses of LeuRS within the Sulfolobaceae

Method I: Sequence gathering and phylogeny generation

Generation of a LeuRS-I antibody

Method I: Transformation and overexpression

Method II: Affinity purification of overexpressed protein

Method III: Thrombin digestion

Generation of a Sulfolobus islandicus genomic C-terminal HA-tagged LeuRS-I

(I-Ct-HA)

Method I: Plasmid Construction

Method II: Transformation of pRJW08-gBlock/D2(+) into wild type S. islandicus

Growth curve of $S$. islandicus LeuRS-I-Ct-HA strain

Expression profiling of HA-I strain using Western blots

Method I: Western blot analysis of fusion protein expression 
Development of a phylogenetic tree comparing LeuRS-F and LeuRS-I in the

Sulfolobaceae

Electrophoretic mobility shift assays

Microscale thermophoresis

tRNA extraction

LeuRS-I C-terminal domain expression and purification

HA-I plasmid construction 


\section{FIGURES}

Figure

Page

1. Comparison of leucyl-tRNA synthetases from E. coli, S. islandicus, and P. Horikoshii

2. A phylogeny consisting of the LeuRS-I and LeuRS-F enzymes across the family Sulfolobaceae

3. A 5\% TBM EMSA gel monitoring the binding of LeuRS-I and $\left[{ }^{32} \mathrm{P}\right]-\mathrm{tRNA}{ }^{\text {Leu }}-\mathrm{UAG}$

4. Acrylamide (37.5:1) pore size and its effect on the binding of LeuRS-F to $\left[{ }^{32} \mathrm{P}\right]-\mathrm{tRNA}{ }^{\mathrm{Leu}}-\mathrm{UAG}$

5. Acrylamide (29:1) pore size and its effect on the binding of LeuRS-F to $\left[{ }^{32} \mathrm{P}\right]-\mathrm{tRNA}^{\mathrm{Leu}}-\mathrm{UAG}$

6. A 5\% bis-tris gel (50\% glycerol supplementation) monitoring the binding of LeuRS-I to $\left[{ }^{32} \mathrm{P}\right]-\mathrm{tRNA}{ }^{\text {Leu }}-\mathrm{UAG}$

7. A $5 \%$ bis-tris gel (20\% TEG) monitoring the binding of LeuRS-I to $\left[{ }^{32} \mathrm{P}\right]-\mathrm{tRNA}{ }^{\mathrm{Leu}}-\mathrm{UAG}$

8. A 5\% bis-tris gel (37.5:1 acrylamide:bisacrylamide) monitoring the binding of LeuRS-I to $\left[{ }^{32} \mathrm{P}\right]-\mathrm{tRNA}{ }^{\mathrm{Leu}}-\mathrm{UAG}$ folded with variable cooling methods

9. 5\% TBM (37.5:1 acrylamide:bisacrylamide) gel stained with SYBR Gold monitoring folded and unfolded cold tRNA ${ }^{\text {Leu }}-\mathrm{UAG}$ in the presence and absence of supplemental buffer

10. 5\% TBM gel stained with SYBR Gold monitoring binding between LeuRS-I and cold tRNA ${ }^{\text {Leu-UAG }}$

11. 5\% TBM gel of LeuRS-I, sans tRNA substrate stained with SYBR Gold

12. An 8\% TBM 75:1 acrylamide:bisacrylamide native PAGE sequencing gel containing serial diluted LeuRS-F, LeuRS-I, and E. coli LeuRS

13. Fluorescence quenching of tryptophan residues through protein-tRNA interaction

14. $12 \%$ sequencing gel analysis of $S i$ wild type total tRNA and tRNA ${ }^{\text {Leu-UAG }}$

15. $12 \%$ sequencing gel analysis of $S i$ wild type total tRNA and E. coli total tRNA 
16. $2 \%$ agarose bleach gel of $E$. coli total tRNA and Si total tRNA

17. Fractionation of total E. coli tRNA from high molecular weight nucleic acids

18. $2 \%$ agarose bleach gel tracking the digestion of $S i$ total tRNA-2 with DNase

19. $2 \%$ agarose bleach gel tracking contamination cleanup in Si total tRNA-2

20. $2 \%$ agarose bleach gel depicting $S i$ total tRNA-4 \& 5 and final processed and pooled low temperature total tRNA

21. Sequence alignment between the C-terminal domain of LeuRS-I (residues 789 934) and LeuRS-F (residues 789 - 944)

22. $15 \%$ SDS-PAGE tracking the affinity purification of LeuRS-I (789 - 934)

23. $15 \%$ SDS-PAGE tracking the thrombin digest of His 6 -LeuRS-I (789 - 934)

24. $15 \%$ SDS-PAGE monitoring the His-tag removal from cleaved LeuRS-I $(789-934)$

25. $15 \%$ SDS-PAGE monitoring the anion exchange purification of cleaved LeuRS-I (789-934)

26. Vector map of the pRJW08-gBlock/D2(+) (HA-I)

27. $1.5 \%$ agarose gel depicting the introduction of the D2 fragment into pRJW08-gBlock $(+)$

28. $1.0 \%$ agarose gel depicting the introduction of the D2 and gBlock fragment within discrete minipreps of the positive pRJW08-gBlock/D2(+) vector (HA-I)

29. Modified MID recombination scheme for the introduction of a C-terminal HA tagged fusion with leuRS-I

30. X-gal staining of $S$. islandicus with integrated HA-I

31. $2 \%$ agarose gel depicting screens of the genomically integrated HA tag in S. islandicus M.16.4

32. Growth curve data of S. islandicus wild type strain and the HA-I strain

33. X-ray film depicting the expression of HA-I across differential temperatures and optical densities 
34. Densitometric intensity counts of LeuRS-I expression at $75^{\circ} \mathrm{C}$ and $60^{\circ} \mathrm{C}$ 


\section{CHAPTER I: INTRODUCTION}

\section{The aminoacyl-tRNA synthetases - an overview}

The aminoacyl-tRNA synthetases (aaRS) are essential enzymes found within all kingdoms of life. These ancient enzymes bridge the worlds of nucleic acids and proteins by catalyzing the addition of an amino acid to their cognate transfer RNA (tRNA) molecule in a two-step reaction. ${ }^{[1]}$ The reaction first begins with the activation of an amino acid via adenosine triphosphate (ATP), forming an adenylated amino acid intermediate with the subsequent release of the byproduct pyrophosphate. ${ }^{[1]}$ Next, the tRNA synthetase catalyzes the formation of an ester linkage between a tRNA and the carboxyl moiety of the activated amino acid, the process of which is commonly referred to as aminoacylation or charging. ${ }^{[2]}$ This linkage forms a stable tRNA - amino acid product (aa-tRNA) that will be used in the synthesis of proteins at the ribosome. ${ }^{[1,2]}$ Secondary carrier elongation molecules within bacteria known as EF1-A and EF1-B perform the task of transporting the newly synthesized aa-tRNA molecule to the ribosome where it will be decoded and used to form stable polypeptide fragments. ${ }^{[3,4]}$ The aminoacyl-tRNA synthetases are broken into two major classes: class I aminoacyl-tRNA synthetases and class II aminoacyl-tRNA synthetases. ${ }^{[5]}$ The major subdivision of these classes of enzyme is defined by both the domain structures of the tRNA binding fold and, accordingly, how the amino acid becomes attached to the tRNA's acceptor stem, with type I aminoacyl-tRNA synthetases possessing a characteristically monomeric Rossman fold binding domain and type II aminoacyl-tRNA synthetases exhibiting multimeric anti-parallel beta-sheets flanked by alternative domains. ${ }^{[5]}$ The aminoacyl-tRNA synthetases have been used to understand the evolutionary similarities and dissimilarities of organisms across the three domains of life. Analysis of the aminoacyl tRNA synthetases has been utilized in tandem with previous work performed by Iwabe and colleagues and, subsequently, 
Brown and Doolittle whom provided strong evidence of close sequence comparation between archaebacteria and eukaryotes, ultimately delineating a general evolutionary affiliation amongst the three domains of life. ${ }^{[6,7]}$

\section{Sulfolobus islandicus, a model hyperthermophilic organism}

The single-celled archaeon Sulfolobus islandicus, of the family Sulfolobaceae and phylum Crenarchaeota, is an extremophilic organism. S. islandicus was initially isolated from areas around the Solfatara volcano in Italy by Brock and cohort ${ }^{[8]}$ This organism is characterized by its ability to oxidize sulfur, to survive through both heterotrophic and chemolithoautotrophic means, and its adapted persistence to harsh conditions, surviving in a habitable temperature range of 65 to $85^{\circ} \mathrm{C}$ and a $\mathrm{pH}$ range of $2-4 \cdot{ }^{[8]}$ The shape of $S$. islandicus cells are lobed spheres that range in size between $0.8-1.0 \mu \mathrm{m}$, typically exhibiting little size variation. ${ }^{[8]}$ Today, many geographically isolated and unique strains of $S$. islandicus have been found in geologically active areas including volcanic vents present in Yellowstone National Park, Iceland, Russia and South America, amongst others. ${ }^{[9]}$ Sulfolobus species have provided a strong platform for understanding archaeal phylogenetics within the crenarchaeal system, in elucidating cellular machinery such as transcription apparatuses and stress-response systems, and have even been used to study cell cycle regulation due to their genetic tractability and ease of culturing in a laboratory setting. ${ }^{[10]}$ More specifically, S. islandicus has been the topic of extensive research in the field of virus-host relationships, DNA replication and repair, biogeographical analyses of pan-genomes within the species, and has been utilized to tailor advanced genetic toolboxes for easy and extensive manipulation within the laboratory setting. ${ }^{[11,12,13,14]}$ 


\section{Duplication of aaRSs' and the act of mistranslation}

With the publication of $S$. islandicus' genome nearly 30 years after the discovery of the organism, unique genes were identified and examined in a paper published by Weitzel and colleagues. ${ }^{[15]}$ Strikingly, S. islandicus was found to possess a conserved duplicated copy of a nonessential leucyl-tRNA synthetase (LeuRS, denoted as LeuRS-I for infidelity) seemingly incapable of performing the standard activity of charging along with the canonical copy of the LeuRS (denoted as LeuRS-F for fidelity) that is essential in performing the charging of leucine onto tRNA ${ }^{\text {Leu }}$, therefore making this protein tantamount to the survival of the cell. ${ }^{[15]}$ The function of LeuRS-I has yet to be determined, while LeuRS-F exhibits canonical aminoacyl-tRNA synthetase activity. LeuRS-I is deemed as having "infidelity" due to a suspected editing deficiency found in the CP1 editing domain (connective polypeptide) of this class I aminoacyl-tRNA synthetases based upon signature sequence analysis, though it has been shown to both activate leucine and bind to tRNA $^{\text {Leu }}{ }^{[15]}$ The function of LeuRS-I has yet to be determined, while LeuRS-F exhibits canonical aminoacyl-tRNA synthetase activity. The CP1 domain of LeuRS-I lacks the universal aspartic acid that is critical in eliciting the hydrolytic activity required for editing mischarged tRNA, while LeuRS-F possesses the canonical hydrolytic aspartic acid in its CP1 domain. ${ }^{[15,16]}$ Editing can occur via two separate mechanisms: pre-transfer and post-transfer editing. Pre-transfer editing occurs when an aminoacyl adenylate (aa-AMP) is formed by the addition of an improper amino acid which is subsequently hydrolyzed in the aminoacylation active site of an aaRS using currently undefined amino acids. This activity within the aminoacylation active site causes the release of the amino acid, thereby allowing the process to start anew. A secondary mechanism known as posttransfer editing resolves the improper mischarging of tRNA with a non-cognate amino acid via a

discrete editing site on the aminoacyl-tRNA synthetase. ${ }^{[17]}$ A double-sieve model first proposed 
by Fresht and Ketner postulated the presence of both a "coarse" sieve or active site that discriminates against amino acids that are too large and thereby incompatible with the given aminoacyl-tRNA synthetase and a "fine" sieve that eliminates mischarged amino acids via editing mechanisms within editing active sites on the enzyme. ${ }^{[18]}$ Though it is unclear as to the utility of LeuRS-I and its non-functional editing domain, proof exists that such a mutation of the hydrolytic aspartic acid to alanine within the CP1 domain of E. coli LeuRS rendered the enzyme incapable of post-transfer editing. ${ }^{[19]}$ The implications of such inactivity within S. islandicus LeuRS-I editing suggests a capacity for mischarging at elevated rates and potentially increasing mistranslation of proteins downstream.

Extensive research has been conducted in a host of organisms aiming to understand mistranslation, which represents itself as both a boon and a detriment depending on the organismal system in question and the specific types of amino acid substitution during protein synthesis. A possibility exists in which LeuRS-I purposefully attaches non-cognate amino acids on to tRNA ${ }^{\text {Leu }}$ or other tRNA isoforms present within the cell under a specific set of required conditions, leading to the incorporation of these improper amino acids into a polypeptide chain. While mistranslation is generalized and at basal levels within many different organisms, the elicitation of heightened levels of mistranslation is often caused by an increase in the stress response of the cell by abiotic or biotic stressors. ${ }^{[20]}$

Degenerate aminoacyl-tRNA synthetases that lack many of the important editing mechanisms of standard aaRS' can be found in a host of different prokaryotic and archael organisms. One such organism, Mycoplasma mobile, possesses degenerated aminoacyl-tRNA synthetases such as LeuRS which is completely devoid of the CP1 editing domain and thus, is incapable of the pre or post transfer editing of improperly charged tRNA's which then go on to 
incorporate translational errors at the ribosome. This leads to possessing "statistical proteomics", meaning these organisms accumulate a reservoir of uniquely substituted proteins that potentially provide phenotypic plasticity and flexibility within the organism. ${ }^{[21]}$ A separate example exists within mammalian cells in the case of methionyl-tRNA synthetase (MetRS). MetRS is capable of attaching methionine to non-cognate tRNA's and can be induced with oxidative stress to perform this mismethionylation at higher percentages, from basal levels of mistranslation at $1 \%$ up to $10 \%$ in cases of stress, with a proposition by the discovering authors of this mechanism as being "adaptive mistranslation."[22]

Speculation exists that the lack of editing capacity in certain aaRSs could be of benefit to an organism under a suite of stressful conditions and has been demonstrated in a variety of bacterial and archaeal species as reported by Ibba and colleague. ${ }^{[23]}$ The hyperthermophilic organism Aeropyrum pernix grows in a temperature range of $70-100^{\circ} \mathrm{C}{ }^{[24]}$ When challenged with the substandard temperature of $75^{\circ} \mathrm{C}, A$. pernix constitutively mischarges methionine onto tRNA ${ }^{\text {Leu }}$ via methionyl-tRNA synthetase. ${ }^{[2]}$ Schwartz and colleague demonstrated that this mistranslation surprisingly heightened the activity of methionine-substituted citrate synthase isolated from cells grown at low temperatures relative to wild type enzyme recovered from cells grown under appropriate conditions. ${ }^{[2]}$ The investigators speculate that mistranslation increases the flexibility and therefore the activity of certain proteins under chilling temperatures. ${ }^{[24]}$ In a recent paper published in PLOS Genetics, researchers found that basal and artificially induced global mistranslation in Escherichia coli enhanced cell survival by increasing the activation of the SOS response under DNA damage during UV radiation, hydrogen peroxide exposure and ciprofloxacin treatments. ${ }^{[25]}$ These findings further support the hypothesis that, while generalized mistranslation under normal conditions can be detrimental to the survival of the cell, mistranslation in response 
to environmental challenge could provide benefits to the organism by altering the amino acid composition of proteins, thereby altering function or capacity of function. ${ }^{[25]}$ Indeed, what was once believed to be a solely negative process within organisms has been scrutinized and opposed given the wide range of experimental data running contrary to original paradigms. As such, adaptive mistranslational mechanisms might be at work within S. islandicus and closely related strains.

A criterion for organismal viability once posited that the presence of twenty aaRSs, one for each of the standard amino acids in a cell, was a standard found in all life. ${ }^{[26]}$ While this is the typical case, examples exist that defy this standard; one such example is exhibited in the prokaryotic species Anabaena sp. PCC 7120. ${ }^{[27]}$ This organism possesses two unique copies of threonyl-tRNA synthetase, one being predominantly active only during times of low zinc availability. ${ }^{[27]}$ This zinc-starvation induced copy contains a defunct editing domain $\mathrm{N} 2$ that significantly impairs post-transfer editing activity and permits the mischarging of serine onto tRNA $^{\text {Thr }}{ }^{[27]}$ Another example of a paralogous tRNA-synthetase exists in the prokaryotic biocontrol species Agrobacterium radiobacter. ${ }^{[28]}$ A. radiobacter produces the antibiotic agrocin 84 which mimics a substrate derived from the plant tumor-inducing species Agrobacterium tumefaciens. ${ }^{\text {[28] }}$ Agrocin 84's mechanism of action is through the inhibition of A. tumefaciens' LeuRS, ultimately halting translation in the organism. ${ }^{[28]}$ A. radiobacter possesses two copies of LeuRS, one of which is resistant to the activity of agrocin 84 , thus preventing self-toxification. ${ }^{[1]}$

Interestingly, the duplication or horizontal transfer of aaRS genes is more frequent than originally predicted and occurred in many prokaryotic and archaeal species. Speculation exists that duplicated forms, while often non-essential, likely confer selective advantages to the host as proposed in the archaeal lineage of the Halobacteriales. ${ }^{[2]}$ The order Halobacteriales of the phylum 
Euryarchaeota possess three unique LeuRS, the first of which was transmitted via an ancient common ancestor of the bacterial domain which was subsequently duplicated or genetically transferred within organisms of the haloarchaea leading to the three distinct forms seen today. The function of the duplicate forms of LeuRS within these organisms is still unclear. ${ }^{[29]}$ The aaRSs are among the more frequent horizontally transferred genes amongst cells in an evolutionary context. ${ }^{[30]}$ As reported by Woese and colleagues, phylogenetic analyses of aaRSs present in a plethora of organisms from the three domains of life revealed a strong pattern of horizontal gene transfer over an ancient and extended timescale. ${ }^{[30]}$

Along with the occurrence of duplicated forms of aminoacyl-tRNA synthetases amongst a wide range of organisms, modified forms of aminoacyl-tRNA synthetases such as tyrosyl-tRNA synthetase (TyrRS) found in higher organisms possess additional domains that have no bearing on the activity of aminoacylation while adding secondary activities that are important to the organism. ${ }^{[31]}$ TyrRS contains two unique domains, tripeptide ELR and EMAPII, which work in tandem within receptor-mediated signaling pathways related to angiogenesis. ${ }^{[31]}$ An example of tryptophanyl-tRNA synthetase (TrpRS) in higher organisms exists by which an addition of a WHEP domain to the synthetase acts as an inhibitor of the potent angiostatic activity of TrpRS within the cell. ${ }^{[31]}$ While no additional domains appear present on LeuRS-I, that does not disclude the enzyme from having novel functionality outside of being a standard aminoacyl-tRNA synthetase, the activity of which has yet to be determined experimentally. At the core of this evolutionary black box, the elucidation of S. islandicus' duplicate LeuRS system is of great importance in determining its relevance and utility in the species. 


\section{Duplication of LeuRS in Sulfolobus islandicus - potential ramifications}

Through the comparison of sequence identity between the two copies of LeuRS present in S. islandicus, it was shown that LeuRS-I shares nearly $40 \%$ amino acid sequence identity with that of LeuRS-F. The C-terminal domains of LeuRS-F and LeuRS-I are even more dissimilar as compared to alignments of the full proteins, approaching only 20\% identity (Figure 1). Some of these variances in sequence are seemingly significant and reside in important areas such as the CP1 domain, perhaps shedding light on the activity of LeuRS-I given that it is capable of both activating leucine and binding tRNA ${ }^{\text {Leu }}$ though it is incapable of charging tRNA ${ }^{\text {Leu }} \cdot{ }^{[15]}$ If LeuRS-I were capable of charging tRNA ${ }^{\text {Leu }}$, it would more than likely be incapable of editing mischarged tRNA leading to downstream mistranslation. This mistranslation leads to amino acids chemically and structurally similar to leucine being substituted at leucine codons. It is possible that LeuRS-I competes with LeuRS-F for substrate given that they have both been shown to be present in the cytoplasm of the cell based upon preliminary ultra-centrifugation and fractionation experiments. This activity between the two enzymes could possibly alter the dynamics of aminoacylation and translation by acting as a resource detection switch that modulates the activity of the two enzymes based upon the levels of aminoacylation resources within the cytoplasm. One such possibility could be the functional relevance of LeuRS-I producing mischarged tRNA which is inherently deacylated by LeuRS-F, the act of which is known as editing in-trans. ${ }^{[32]}$ Under a certain set of conditions, this activity of trans editing could potentially be elicited by LeuRS-F in the presence of misacylated tRNA ${ }^{\text {Leu }}$ generated by LeuRS-I while a secondary condition leaves these improperly charged tRNA's unfettered. For instance, if LeuRS-I were capable of charging leucine on to a non-cognate tRNA, LeuRS-F should be incapable of binding to this non-cognate tRNA and 
therefore be unable to edit in-trans, with the subsequent downstream effect of mistranslation occurring. 


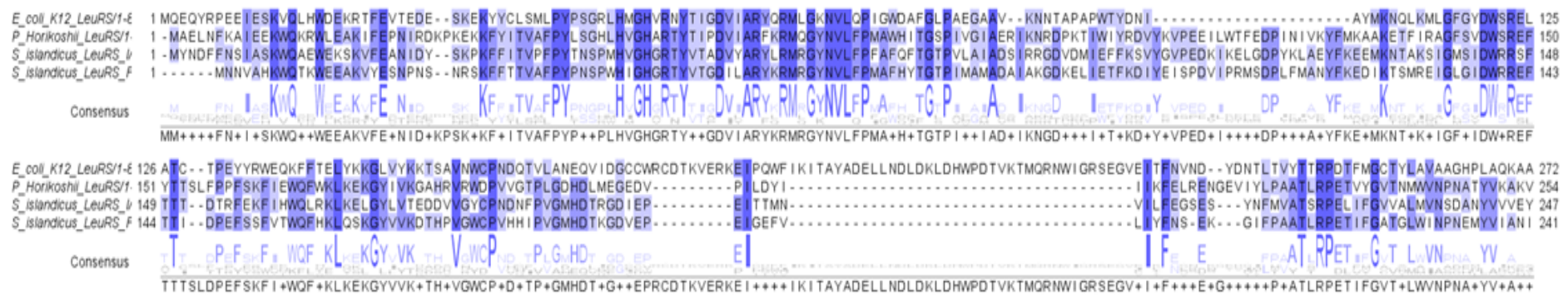

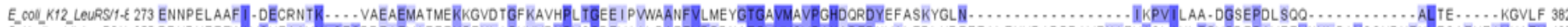

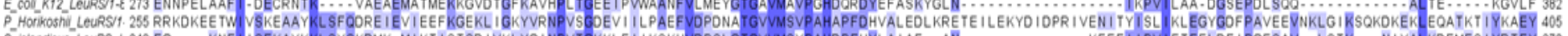

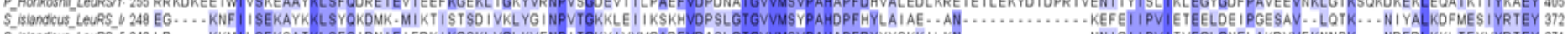

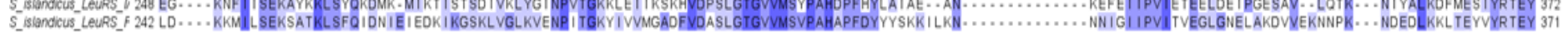

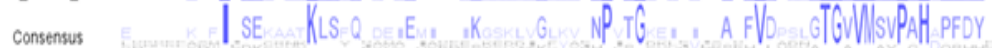
IIPV| EOG P V V K K L L YY EY

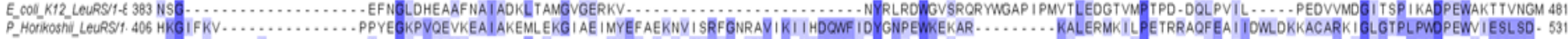

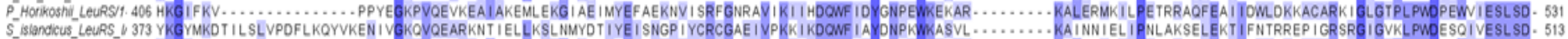

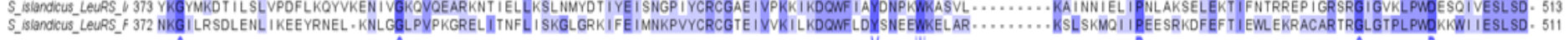
Consensus

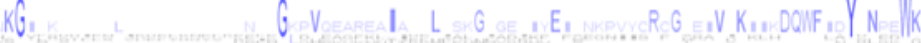

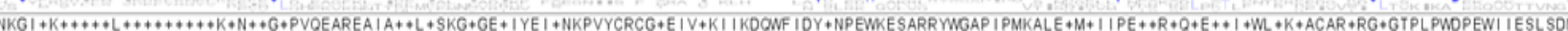

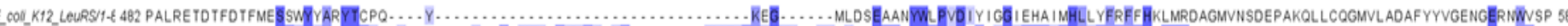

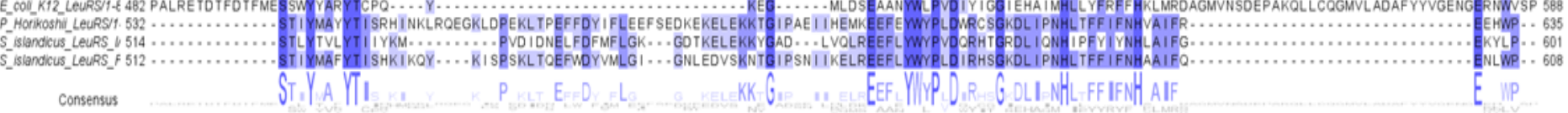

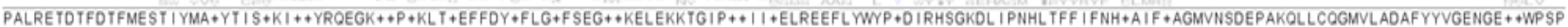

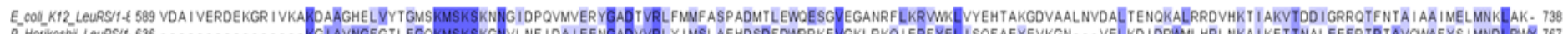

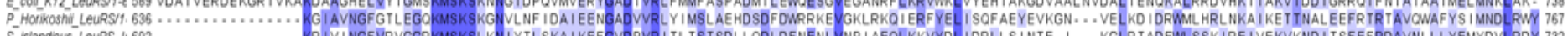

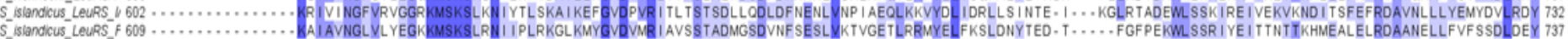

Consensus IA NG V G KISIS N

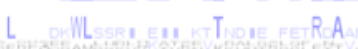

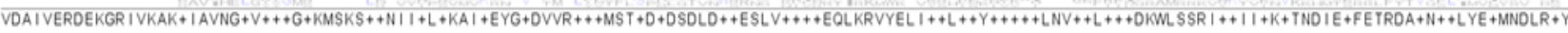

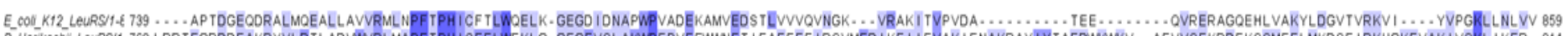

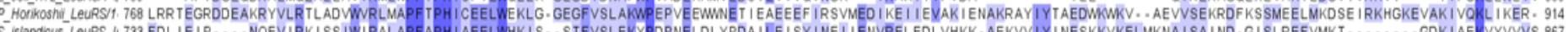

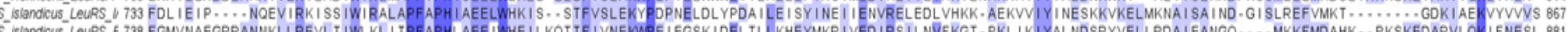

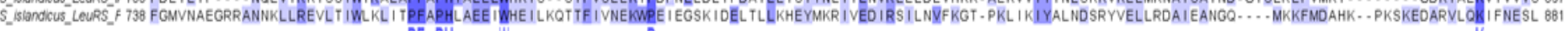
Consensus PF PI EELII. I

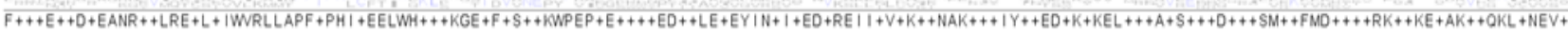

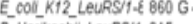

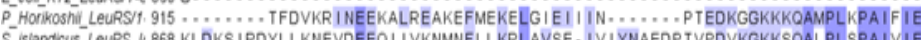

S_islandicus_LLURS I 868 KLDKS IRDYLLKNEVDEEQII IKNMNFLLKRLAVSE. IVI YNAEDPTVPDVKGKKSQALPLSPAIVIE

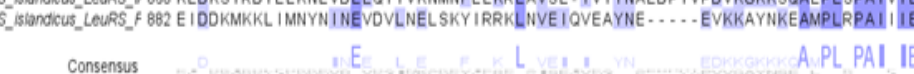

Consensus A.PL PAI IE

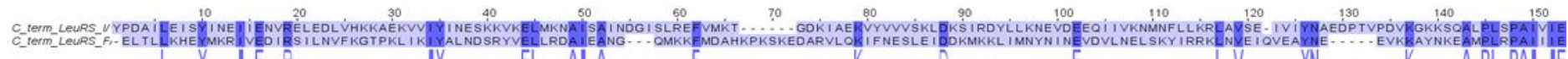

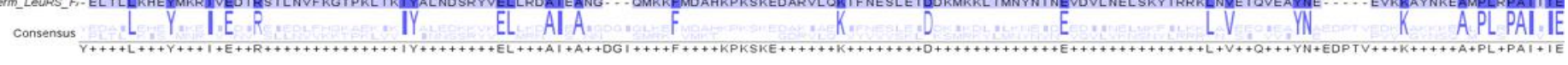

Figure 1: Comparison of leucyl-tRNA synthetases from E. coli, S. islandicus, and P.

horikoshii, along with C-terminal domain comparison. Synthetases aligned using Clustal Omega.

Greatest divergence of sequence identity can be seen between the C-terminal domains of LeuRS-

F and LeuRS-I with consensus data shown. 


\section{Summary of research objectives}

The elucidation of LeuRS-I's function in S. islandicus is the utmost priority of our research project. The production of molecular tools that can aid in the analysis or detection of LeuRS-I both in vitro and in vivo will help us accomplish our goals of determining the presence and amount of this paralog in S. islandicus throughout its growth phases during favorable and challenging environmental conditions. These tools include generating polyclonal antibodies that are selective in their recognition of LeuRS-I along with the production of an epitope-tagged genomic LeuRS-I for downstream experimentation. Both of these developed tools would provide methods for tracking in vivo expression levels of LeuRS-I, and speculatively, yield one of three outcomes: 1) LeuRS-I is found to be constitutively expressed, 2) its presence in S. islandicus is temporally controlled and is only expressed during certain stages of growth, or 3) LeuRS-I is only expressed during unfavorable or stressful growth conditions. With the previous information in mind, a variety of different conditions with which to utilize these tools can be employed as treatments to elicit potential expression levels of this enzyme and detect its presence.

If the presence of $S$. islandicus' LeuRS-I cannot be detected under standard growth conditions, low temperatures that would be considered a harsh environmental challenge to the organism could be employed to elicit its detection. Along these lines, changes in the $\mathrm{pH}$ of $S$. islandicus' media could also be adopted as a stressor in an attempt to detect LeuRS-I in vivo. Following this rationale, an example of a similar scenario is observed in discrete temperaturedependent, $\mathrm{pH}$ inducible forms of lysyl tRNA-synthetase (LysRS) from E. coli K-12 MG1655. ${ }^{[33]}$ Two copies of a gene encoding for LysRS exist in the E. coli K-12 genome, denoted as lysS and lys $U$, respectively. ${ }^{[33]}$ The lys $S$ gene is found to be constitutively active at standard conditions while transcription of the lys $U$ gene is induced at $\mathrm{pH}$ 's of 5.5 or lower, with temperatures below 
$37^{\circ} \mathrm{C}$ affecting the induction of this gene negatively. ${ }^{[33]}$ If the efficacy of LeuRS-I specific antibodies and a C-terminal HA-tagged LeuRS-I can be verified, a variety of experimental parameters can be employed in determining the presence and relative abundance of LeuRS-I in $S$. islandicus cells. 


\section{CHAPTER II: EXPERIMENTAL METHODS}

\section{tRNA Extractions}

Methods for extracting tRNA from both Escherichia coli (E. coli) and S. islandicus were developed in the Weitzel laboratory with the intention of obtaining reproducible and consistent yields of tRNA for downstream applications. Several trials were conducted as described below.

\section{Method I: Small-scale extraction of total $S$. islandicus tRNA}

Wild type and leuRS-I knockout cultures were started from freezer stocks by inoculating 2 $\mathrm{mL}$ of DT/AU media consisting of $0.3 \% \mathrm{~K}_{2} \mathrm{SO}_{4}, 0.05 \% \mathrm{NaH}_{2} \mathrm{PO}_{4}, 0.0145 \% \mathrm{MgSO}_{4}, 0.01 \%$ $\mathrm{CaCl}_{2} \cdot 2 \mathrm{H}_{2} \mathrm{O}, 0.1 \%$ Dextrin, 0.1\% EZMix N-Z-Amine A, $20 \mu \mathrm{g} / \mathrm{mL}$ uracil, $20 \mu \mathrm{g} / \mathrm{mL}$ agmatine, $0.00006 \% \quad \mathrm{FeCl}_{3}, \quad 0.00001 \% \mathrm{CoCl}_{2} \bullet 6 \mathrm{H}_{2} \mathrm{O}, 0.00001 \% \quad \mathrm{MnCl}_{2} \bullet 4 \mathrm{H}_{2} \mathrm{O}, 0.00001 \% \mathrm{ZnCl}_{2}$, and $0.00001 \% \mathrm{CuCl}_{2} \cdot 2 \mathrm{H}_{2} \mathrm{O}(\mathrm{wt} / \mathrm{vol})$ with $100 \mu \mathrm{L}$ of semi-frozen stock. Drams were allowed to incubate at $75^{\circ} \mathrm{C}$ for two days. Noting cloudiness, $1 \mathrm{~mL}$ of these starter cultures were then passaged into $20 \mathrm{~mL}$ of fresh DT/AU (passage 1 cells) and allowed to grow at $75^{\circ} \mathrm{C}$ until an optical density at $600 \mathrm{~nm}\left(\mathrm{OD}_{600}\right)$ of approximately 0.2 ( three days). Both samples were then passaged a second time into $100 \mathrm{~mL}$ of fresh DT/AU media, standardizing initial $\mathrm{OD}_{600}$ s to 0.008 . Samples were, again, grown at $75^{\circ} \mathrm{C}$ until each reached $\mathrm{OD}_{600 \mathrm{~s}}$ of approximately 0.3. Cells were harvested via centrifugation at $8,000 \times \mathrm{g}$ for 30 minutes at $4^{\circ} \mathrm{C}$. Spent DT/AU medium was discarded and cell pellets were placed on ice.

For the initial lysis, pellets were resuspended in $1 \mathrm{~mL}$ of $\mathrm{LiCl}$ reagent $(2.5 \mathrm{M} \mathrm{LiCl}, 50 \mathrm{mM}$ Tris- $\mathrm{HCl} \mathrm{pH} 8.0,4 \%$ triton $\mathrm{X}-100,62.5 \mathrm{mM}$ EDTA $\mathrm{pH} 8.0$ ). The $\mathrm{LiCl}$ salt is primarily used in extractions that require the inclusion of small RNA's due to their solubility in the reagent, while large RNA is readily excluded due to their insolubility in this reagent. ${ }^{[34]}$ An equal volume of acid phenol:chloroform:isoamyl alcohol (Ambion, P:C:I 125:24:1), $\mathrm{pH} 4.5$ was added to each cellular 
resuspension followed by vigorous vortexing to further homogenize. Samples were then incubated on ice for 10 minutes.

After incubation, the lysates were transferred to $15 \mathrm{~mL}$ falcon tubes and centrifuged at $13,500 \mathrm{x} \mathrm{g}$ for 10 minutes at $4^{\circ} \mathrm{C}$. After centrifugation, a visibly thick plug was present in each sample, likely containing membrane and denatured protein, that occluded the aqueous layer from migrating to the top of the extraction. To remedy this issue, $0.5 \mathrm{~mL}$ of each reagent (now currently $1.5 \mathrm{~mL}$ for the updated protocol), or 1:1 $\mathrm{LiCl}$ and $\mathrm{P}: \mathrm{C}: \mathrm{I}$ solution, was added to each, mixed vigorously, and re-centrifuged to separate the layers. These additional steps resulted in wellresolved aqueous layers that were collected and transferred to fresh $15 \mathrm{~mL}$ falcon tubes. In an effort to remove residual phenol, $1.6 \mathrm{~mL}$ pure chloroform was added to both samples and mixed vigorously. Subsequently, samples were centrifuged at $13,500 \mathrm{x}$ g for 10 minutes at $4^{\circ} \mathrm{C}$, afterwhich roughly $1.5 \mathrm{~mL}$ of a top aqueous phase was collected. This crude tRNA-containing solution was transferred to a $15 \mathrm{~mL}$ conical tube.

To initiate precipitation of extracted RNAs, sodium acetate (NaOAc), pH 5.2 was added to the crude tRNA to a final concentration of $0.3 \mathrm{M}$ prior to the addition of 2.5 volumes of $-20^{\circ} \mathrm{C}$ absolute ethanol. The samples were gently mixed and placed at $-20^{\circ} \mathrm{C}$ overnight. After this overnight incubation, samples contained tendrils of white precipitate. Precipitates were collected via centrifugation at $13,500 \mathrm{x}$ g for 10 minutes at $4^{\circ} \mathrm{C}$. Resulting supernatants covering visible pellets of nucleic acid and/or salt were discarded. Pellets were rinsed twice with $2 \mathrm{~mL}$ of $70 \%$ ethanol, while also ensuring that the sides of the tube were rinsed to remove residual NaOAc or LiCl. To ensure that the pellets were retained, between and after the final rinse, samples were centrifuged at $13,500 \mathrm{x}$ g for 10 minutes at $4{ }^{\circ} \mathrm{C}$. To remove residual ethanol, rinsed pellets were allowed to dry in a laminar flow hood for 10 minutes. Pellets were resuspended in $15 \mu \mathrm{L}$ Ambion 
elution buffer (AEB, $10 \mathrm{mM}$ Tris $\mathrm{pH}$ 7.0, $0.1 \mathrm{mM}$ EDTA). After the pellets were completely dissolved, nucleic acid concentration (absorbance at $260 \mathrm{~nm}$ ) and quality $(260 \mathrm{~nm} / 280 \mathrm{~nm}$ and 260 $\mathrm{nm} / 230 \mathrm{~nm}$ ratios) were assessed using a Nanodrop One (Fisher Scientific) using the RNA protocol.

To assess the quality of the crude tRNA extracts, the samples were analyzed via $12 \%$ polyacrylamide sequencing gels. A $12 \%$ polyacrylamide, $7 \mathrm{M}$ urea solution was made by mixing solutions of $1 \mathrm{x}$ TBE/7M urea $[89 \mathrm{mM}$ Tris $\mathrm{pH}$ 7.6, $89 \mathrm{mM}$ boric acid, $2 \mathrm{mM}$ ethylenediaminetetraacetic acid (EDTA)] and 20\% acrylamide (19:1) in 1x TBE/7M urea. The polymerization reaction was initiated with $10 \%$ ammonium persulfate (APS, $0.0064 \% \mathrm{vol} / \mathrm{vol}$ ) and tetramethyelthylenediamine (TEMED, 0.0019\% vol/vol). Gel samples were prepared by adding RNA loading buffer to a 1x final concentration [0.175 M Urea, 10 mM EDTA pH 8.0, 0.000025\% xylene cyanol (wt/vol) and $0.000025 \%$ bromophenol blue (wt/vol)] to $2000 \mathrm{ng}$ of diluted crude wild type and knockout tRNA. An in vitro transcribed Si tRNA ${ }^{\mathrm{Leu}}-\mathrm{UAG}$ isoacceptor (2000 ng) generated within the lab was used as a control sample for comparison to endogenously extracted total tRNA. Gels were run in $1 \mathrm{x}$ TBE at a constant current of $20 \mathrm{~mA}$ resulting in a steady voltage of approximately $1250 \mathrm{~V}$. If the voltage increased dramatically, the current was iteratively decreased to reestablish the desired $1250 \mathrm{~V}$. Gels were stopped when the xylene cyanol dye band was $2-3 \mathrm{~cm}$ from the bottom.

The developed extraction protocol was applied to the isolation of total Escherichia coli tRNA to assess its broader applicability. Five milliliters of Lysogeny broth (LB, $1 \%$ Typtone w/v, $1 \% \mathrm{NaCl} w / \mathrm{v}, 0.5 \%$ Yeast extract $\mathrm{w} / \mathrm{v}$ ) was placed into a glass culture tube and inoculated with 50 $\mu \mathrm{L}$ of $E$. coli BL21 freezer stock. The inoculated medium was incubated in an incubator-shaking (incu-shaker) at $37^{\circ} \mathrm{C}$ and $200 \mathrm{rpm}$ overnight. The next day, the culture tube contained noticeably 
thick growth. This starter culture was used to inoculate $30 \mathrm{~mL}$ of LB at an initial $\mathrm{OD}_{600}$ of 0.008 prior to incubating at $37{ }^{\circ} \mathrm{C}$ with aeration $(200 \mathrm{rpm})$. The culture was monitored over the course of 6 hours. Cells were harvested via centrifugation $\left(15,000 \mathrm{x} \mathrm{g}, 15 \mathrm{~min}, 4^{\circ} \mathrm{C}\right)$ after reaching an $\mathrm{OD}_{600}$ of 1.4. Total tRNA was extracted as previously described, though the requirement of additional $\mathrm{LiCl}$ and $\mathrm{P}: \mathrm{C}: \mathrm{I}$ was unnecessary due to proper separation of the aqueous and organic phases following centrifugation. The resulting tRNA pellet was allowed to dry in the laminar flow hood for 10 minutes and was subsequently resuspended in $100 \mu \mathrm{L}$ of AEB. The tRNA concentration and quality were assessed using the NanoDrop One (Thermo Fisher) and its RNA protocol.

\section{Method II: Large-scale extraction of total S. islandicus tRNA}

As assessed by PAGE, limited quantities of properly sized RNA were obtained using the small-scale extraction protocol. Therefore, isolation of total Si tRNA was attempted on a large scale. Passage 1 cells were allowed to grow in $20 \mathrm{~mL}$ of DT/AU media at $75^{\circ} \mathrm{C}$ to a final $\mathrm{OD}_{600}$ of 0.370 (three days). Four 2.5 L Fernbach flasks, each containing $250 \mathrm{~mL}$ of DT/AU media pH 3.5, were inoculated with $4.5 \mathrm{~mL}$ of passage 1 cells to a starting O.D of 0.008 . The inoculated flasks were placed in the $75^{\circ} \mathrm{C}$ incubator and grown for 5 days. Optical densities at $600 \mathrm{~nm}$ were obtained for each flask and measurements ranged from 0.191 to 0.317 . Cells were harvested via centrifugation at $4250 \mathrm{rpm}\left(45 \mathrm{~min}\right.$ at $\left.4^{\circ} \mathrm{C}\right)$. Cell pellets were processed as described for Method I extractions except using initial $\mathrm{LiCl}$ reagent and $\mathrm{P}: \mathrm{C}: \mathrm{I}$ volumes of $2 \mathrm{~mL}$ each. After centrifugation, a thick cream-colored layer was present in the tube, again occluding the aqueous layer from separating from the organic layer. An additional $1 \mathrm{~mL}$ of $\mathrm{LiCl}$ was added to the tube, followed by brief bouts of vigorous vortexing and additional centrifugation. As before, these additional steps yielded a well-resolved aqueous layer. This crude total tRNA-containing fraction 
was collected, and the nucleic acids were precipitated using $\mathrm{NaOAc/ethanol} \mathrm{as} \mathrm{previously}$ described. The resulting crude, total tRNA pellet was dried in the laminar flow hood for 15 minutes and resuspended in $50 \mu \mathrm{L}$ of AEB. Sample was tested for concentration and purity via the Nanodrop One and its RNA protocol.

A leuRS-I knockout strain has a characteristic lag-in-growth phenotype at sub-optimal temperatures for $S$. islandicus. A reasonable interpretation is that LeuRS-I activity is more important to S. islandicus under these stressful conditions. Additionally, LeuRS-I may need a tRNA substrate containing a modification found only under these conditions. Therefore, to isolate tRNAs that may contain such alterations, a second, large-scale total tRNA extraction of wild type S. islandicus cells was performed with the following modifications. Passage 1 cells were processed as described. At the optimal $\mathrm{OD}_{600}$ of 0.380 , cells were harvested and diluted into two flasks, each containing $500 \mathrm{~mL}$ of fresh DT/AU medium, at an initial OD 600 of 0.008 . Inoculated medium grew at $60^{\circ} \mathrm{C}$ with aeration $(230 \mathrm{rpm})$ until $\mathrm{OD}_{600}$ readings of approximately 0.340 and $0.470(\sim 6$ days $)$ were obtained. Cells were harvested and total tRNA was extracted as described except each pellet originating from $500 \mathrm{~mL}$ of culture was processed individually using $2.0 \mathrm{~mL}$ of $\mathrm{LiCl}$ and $2.0 \mathrm{~mL}$ of P:C:I. Additionally, insoluble/solvent phases resulting from the initial extraction were back extracted with $2 \mathrm{~mL}$ of additional $\mathrm{LiCl}$ to recoup additional RNA. Because of this $4 \mathrm{~mL}$ of chloroform was used to remove residual phenol from the combined aqueous phases. RNA pellets obtained after $\mathrm{NaOAc} / \mathrm{EtOH}$ precipitations were resuspended in $50 \mu \mathrm{L}$ of $\mathrm{AEB}$. 


\section{Method III: Agarose bleach gel electrophoresis}

Due to the laborious nature of pouring, running, and developing sequencing polyacrylamide gels to observe tRNA quality, a secondary method of electrophoretic analysis was implemented following the work of Aranda and colleagues. ${ }^{[35]} 2 \%$ bleach gels were made by adding $2 \mathrm{~g}$ of agarose LE (Goldbio) to $100 \mathrm{~mL}$ of $1 \mathrm{x}$ tris base, acetic acid and EDTA solution (TAE, $40 \mathrm{mM}$ Tris base, $20 \mathrm{mM}$ Acetic acid, $1 \mathrm{mM}$ EDTA). The agarose slurry was mixed thoroughly and microwaved at $80 \%$ power for 1 minute, mixed again, and then microwaved in 30 second increments at $80 \%$ power until fully dissolved. To this, $1 \mathrm{~mL}$ of bleach (Clorox) was added followed by brief microwaving at $80 \%$ power. Ethidium bromide was added to the solution at a final concentration of $0.5 \mu \mathrm{g} / \mathrm{mL}$ followed by 20 seconds of microwaving at $80 \%$ power. tRNA samples were diluted to $1 \mu \mathrm{g} / \mu \mathrm{L}$. Dilutions were mixed 1:1 (vol:vol) with 2x NEB ssRNA loading dye $(47.5 \%$ formamide, $0.01 \%$ SDS, $0.01 \%$ bromophenol blue, $0.005 \%$ xylene cyanol, $0.5 \mathrm{mM}$ EDTA). $2 \mu \mathrm{g}$ of RNA samples were loaded unless otherwise indicated. Low-range ssRNA ladder (NEB) was prepared for electrophoresis by adding $2 \mu \mathrm{L}$ of low-range ssRNA ladder to $2 \mu \mathrm{L}$ of $2 \mathrm{x}$ loading dye. Agarose bleach gels were run for 30 minutes at a constant voltage that equated to 90 $\mathrm{mA}$, starting from $100 \mathrm{~V}$ and ending at $87 \mathrm{~V}$. Typically, voltage was attenuated twice to ensure 90 mA were maintained. The gel was assessed on a UVP GelDoc-It system.

\section{Method IV: Small RNA enrichment}

Bleach gels routinely revealed the presence of larger nucleic acid contaminants within extraction solutions. These larger nucleic acid species were assumed to be primarily genomic DNA (gDNA), ribosomal RNA, and larger mRNA species. To remove larger RNA species, an additional precipitation with $\mathrm{LiCl}$ reagent was utilized. Crude total tRNA was added 1:1 with $\mathrm{LiCl}$ reagent (1.25 M LiCl, $25 \mathrm{mM}$ Tris-HCl pH 8.0, 2\% triton X-100, $31.25 \mathrm{mM}$ EDTA pH 8.0 final 
concentrations), followed by gentle mixing. Large RNAs were allowed to precipitate from these solutions for $10 \mathrm{~min}$ on ice. Precipitations were subsequently centrifuged at $16,000 \mathrm{x}$ g for $20 \mathrm{~min}$ at $4^{\circ} \mathrm{C}$. Following this, samples routinely contain a visible pellet. The supernatant, now enriched for smaller RNAs, was collected and precipitated overnight at $-20^{\circ} \mathrm{C}$ using $\mathrm{NaOAc/ethanol} \mathrm{as}$ already described. The resulting pellets were rinse with $70 \%$ ethanol twice, allowed to dry, and resuspended in $16 \mu \mathrm{L}$ of AEB. Pellets resulting from the large RNA precipitation were also rinsed twice with $70 \%$ ethanol, allowed to dry, and resuspended in $50 \mu \mathrm{L}$ AEB. The resulting RNA solutions (large and small) were assessed for concentration and purity as described. RNase free DNase I (NEB) was then used to remove gDNA from the total small RNA. Reactions were constructed as recommended by the manufacturer and were allowed to incubate at $37^{\circ} \mathrm{C}$ for $1 \mathrm{hr}$. Digested samples were precipitated, rinsed, and resuspended in AEB. All samples were visualized via agarose-bleach gels.

\section{Electrophoretic mobility shift assays and Microscale thermophoresis}

To understand the capacity for LeuRS-I to bind tRNA ${ }^{\text {Leu }}$ molecules, both electrophoretic mobility shift assays (EMSA) and microscale thermophoresis (MST) were employed.

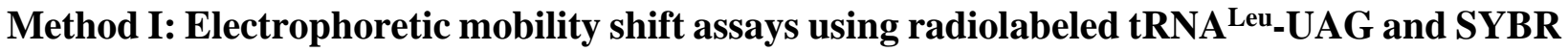 Gold}

A reliable buffering system for running high quality polyacrylamide gels was required for reproducible results within EMSA experiments. The major buffering system initially tested to assess the quality of sample electrophoresis was a 3-( $N$-morpholino)propanesulfonic acid (MOPS) system and a tris/borate/magnesium (TBM) system. Stock buffers at a $5 \mathrm{x}$ concentration were made and diluted to $1 \mathrm{x}$ (MOPS - $100 \mathrm{mM}$ MOPS free acid, $25 \mathrm{mM}$ sodium acetate, $5 \mathrm{mM}$ EDTA, 5mM 
$\mathrm{MgCl}_{2}$; TBM - $89 \mathrm{mM}$ Tris base, $200 \mathrm{mM}$ boric acid, $5 \mathrm{mM} \mathrm{MgCl} 2$ ). These buffering systems were consistent throughout EMSA experiments unless otherwise noted.

Along with the different buffering systems, two major polyacrylamide gel (PAG) systems were tested for suitability within EMSA experiments. Gel compositions varied as experimental conditions were panned. Initial gel systems included a tris-borate (TB) and a bis-tris methane (bistris) buffered PAG environment. Bis-tris gels were tested at pHs of 7.6 and 6.5. Initial EMSA gels were comprised of $0.5 \mathrm{x}$ TB buffer (44.5 $\mathrm{mM}$ tris base, $100 \mathrm{mM}$ borate), $5 \%$ acrylamide (37.5:1 acrylamide:bisacrylamide vol/vol), $2 \%$ glycerol (vol/vol), and $15 \mathrm{mM} \mathrm{MgCl}$. Acrylamide polymerization was induced with the addition of $0.001 \%$ APS (vol/vol) and $0.001 \%$ TEMED (vol/vol) after taking the gel solution to $15 \mathrm{~mL}$ with $\mathrm{ddH}_{2} \mathrm{O}$. Equivalent Bis-tris gels ( $\mathrm{pH} 7.6$ and 6.5) were also utilized using the same concentrations of reagents.

Previously generated 5, $\left[{ }^{32} \mathrm{P}\right]-\mathrm{Si}$ tRNA ${ }^{\mathrm{Leu}}-\mathrm{UAG}$ isoacceptor was utilized as substrate for assessing the ability of LeuRS-I to bind tRNA via EMSA. A 5x supplemental buffer was made to produce a suitable environment conducive for binding between LeuRS-I and tRNA ${ }^{\text {Leu-UAG, with }}$ a $5 \mathrm{X}$ reaction consisting of $295 \mu \mathrm{M}$ MES, $\mathrm{pH} 6.7,75 \mu \mathrm{M} \mathrm{ZnSO}_{4}$ and $20 \mu \mathrm{M} \mathrm{NaCl}$. A 10X tRNA folding mix was assembled with final $200 \mathrm{nM} 5^{\prime}\left[{ }^{32} \mathrm{P}\right]-S i$ tRNA ${ }^{\mathrm{Leu}}-\mathrm{UAG}$ (hot UAG) and $300 \mathrm{nM}$ non-radiolabeled tRNA ${ }^{\mathrm{Leu}}-\mathrm{UAG}$ (cold UAG) $5 \mathrm{mM} \mathrm{MgCl}_{2}, 10 \mathrm{mM}$ MES and was taken to a final volume of $100 \mu \mathrm{L}$ with $\mathrm{dH}_{2} \mathrm{O}$. Water was brought to a vigorous boil and the temperature was monitored using a thermometer. When the water bath reached $93^{\circ} \mathrm{C}$, the previously assembled $10 \mathrm{X}$ tRNA mix was inserted for three minutes. Subsequently, the waterbath, now containing the tRNA mix, was placed on ice and allowed to cool to room temperature. Ten PCR tubes were placed in series, the first of which contained $10 \mu \mathrm{L}$ of $8.8 \mu \mathrm{M}$ LeuRS-I to assess the coherence of EMSA. All other tubes in series were aliquoted with $5 \mu \mathrm{L}$ of protein storage buffer and were two fold 
diluted with an initial volume of $5 \mu \mathrm{L}$ LeuRS-I at $8.8 \mu \mathrm{M}$. From these tubes, $4 \mu \mathrm{L}$ of protein/buffer mix was removed and combined with $2 \mu \mathrm{L}$ of $10 \mathrm{X}$ folding mix and $2 \mu \mathrm{L}$ of $\mathrm{dH}_{2} \mathrm{O}$, then was allowed to incubate at room temperature for 30 minutes. Following incubation, $2 \mu \mathrm{L}$ of bromophenol blue dye (360 mM MES pH 6.5, 60\% glycerol, $0.00001 \%$ bromophenol blue) was added to the reactions. Prior to sample electrophoresis with a TBM system (5X buffer stock at $\mathrm{pH} 7.6$ ), a bistris gel was made using the previously mentioned recipe and was pre-run without samples in prechilled 0.5X TBM buffer on ice for 40 minutes at $100 \mathrm{~V}$, afterwhich the total volume of each sample was loaded and electrophoresed until the dye front approached the end of the plate. The gel was immediately removed from the apparatus and dried on a Gel-drying system (Biorad Model 583 gel dryer) and placed in a FUJIX BAS casette 2040 phosphorscreen (Fujifilm Medical Systems U.S.A., Inc.) for four hours. The phosphor screen was removed and scanned using a GE Typhoon FLA 7000.

Due to a lack of shift appearing within the previously run TBM gel and an apparent lack of migration of LeuRS-I into the gel during electrophoresis, a new round of EMSA was run using the secondary MOPS buffering system with bis-tris gels, as well as varied acrylamide:bisacrylamide ratios in an attempt to aid in the migration of protein into the gel. Samples of protein and tRNA were worked up using the previously mentioned protocol of serial dilution and were composed of the same concentrations of reagents within both the folding mix and supplemental buffers. However, LeuRS-F was substituted in place of LeuRS-I as a control with the expectation of a clear shift being seen. Unlike the previously assembled samples, the final two tubes of the dilution series comprised of both a tRNA control devoid of protein and a tube containing LeuRSF plus I-Ct antibody developed within the Weitzel laboratory, with the intention of possibly seeing a supershift between the antibody and the protein. Reaction preparation followed the previous 
methods and utilized the same bis-tris gel system, however the gel compositions varied in ratios of acrylamide:bis-acrylamide, with one gel containing a ratio of 29:1 and 37.5:1, respectively. Gels were electrophoresed and imaged using the previously mentioned protocol.

Due to the jagged nature of the electrophoretic quality of the MOPS system with the bistris gels, it was determined that the gel composition may need to be attenuated to result in a more consistent migration of sample. Because both the 29:1 and 37.5:1 acrylamide gels had the same quality of jaggedness in band electrophoresis, it was determined that perhaps the glycerol in the sample was effecting this. A $20 \%$ triethyleneglycol (TEG) solution was substituted in place of the $50 \%$ glycerol solution that was originally added to the gels due to its ability to stabilize nucleic acid-protein complexes during electrophoresis as noted by Sidorova and cohort. ${ }^{[36]}$ All other gel components were maintained and used a standard 37.5:1 acrylamide solution. As compared to the last experiment, all methods were maintained aside from $20 \%$ TEG or $50 \%$ glycerol addition to the gel composition. LeuRS-I was used as the protein within these reactions.

Because of the lack of a discernable shift within EMSA using LeuRS-I and LeuRS-F, other parameters of the reaction were augmented. One theory regarding the lack of gel shifting postulated the possibility of improper folding of the tRNA molecules using the standard heating and cooling method in boiling water, which was then modified experimentally. Utilizing the same supplemental buffers as previously used, it was determined that two separate folding mixes should be made, one of which would be heated and cooled using the same parameters as previously mentioned, with the other being immediately flash-cooled after the initial 3 minutes of heating in near-boiling water. Unlike the other protocols utilizing a ten tube serial dilution series, these samples were only aliquoted into three tubes, the first of which was straight LeuRS-I protein, a two-fold and a three-fold dilution, all of which were combined with supplemental buffer and the 
respective folding mixes. Samples were electrophoresed using the MOPS buffering system and a bis-tris gel of standard makeup, as previously mentioned.

A common theme within previously run EMSA reactions was the large amount of streaking, or tails, that would be seen in the gels. This streaking patterns' eminence within the gels proved to be very difficult to quell in all experimentation utilizing the hot UAG substrate, of which a possibility existed in which degradation products of the hot UAG could be adding noise to the shift assays. Therefore, it was determined that a radiation-free substrate would be required to assess the quality of EMSA. The proprietary dye SYBR® Gold (Thermo Fisher Scientific) was utilized as a safe and radiation-free alternative in assessing EMSA reactions. ${ }^{[37]}$ SYBR Gold dye binds with high fidelity to RNA and could be used in assessing any interaction between LeuRS-I and tRNA molecules. ${ }^{[37]}$ The stain was tested initially with varied concentrations of cold UAG to assess the sensitivity and quality of staining of tRNA, as well as the quality of in-vitro transcribed tRNA ${ }^{\text {Leu }}-U A G$ isoacceptor. TBM gels were assembled under standard procedure and both folded and unfolded samples of cold UAG were generated. A condition of supplemental buffer addition and subtraction from the samples was also used to assess quality of the tRNA's environment and the effect of the supplemental buffer on the electrophoresis quality. Using the standard concentrations of reagents within the supplemental buffers, the cold UAG was aliquoted in varied concentrations and reagent conditions. A total of $12 \mu \mathrm{L}$ of sample was loaded on a TBM gel and was electrophoresed under standard conditions. SYBR Gold was diluted 1:100 in a 1X TAE buffer, and the gels were stained for 30 minutes.

Due to the quality of the SYBR Gold treatment on the cold UAG substrate, it was determined that EMSA utilizing LeuRS-I was feasible. Samples were prepared under the standard procedure of serial dilution and contained the original folding mix reagents as well as supplemental 
buffer reagents. The final dilution tube in series contained a tRNA control devoid of protein. Samples were run using the MOPS / bis-tris system and were stained using SYBR Gold at the previously mentioned concentrations and times.

In determining the quality of the shift between the cold UAG and LeuRS-I, the expected decrease in signal of the lower tRNA bands within the higher concentration protein lanes was not easily seen. Therefore, it was determined that a control gel in which only protein was loaded could untangle whether the SYBR Gold reagent was specific to binding only the tRNA in the sample, or if it is binding to the protein as well and giving a "false shift". The process of working up the dilutions of protein was repeated as previously mentioned but lacked the addition of folding mix and tRNA to the samples. The samples were run using the same MOPS / bis-tris system and were electrophoresed and stained using the sample protocol as previously mentioned.

The seeming lack of migration of the protein samples into the acrylamide gels, either utilizing the bis-tris or the TBM gels, had yet to be alleviated. A custom-made sequencing TB acrylamide gel (75:1 acrylamide:bis-acrylamide), was utilized to determine the migrational properties of LeuRS-I, LeuRS-F, and a control E. coli LeuRS. Samples were serial diluted using protein storage buffers with initial protein concentrations of LeuRS-F at $160 \mu \mathrm{M}$, LeuRS-I at 20 $\mu \mathrm{M}$, and $E$. coli LeuRS at $86 \mu \mathrm{M}$. A total of $20 \mu \mathrm{L}$ of each sample was loaded on a $22 \times 20$ short plate TBM sequencing gel with $0.4 \mathrm{~mm}$ spacers and was electrophoresed for 8 hours at $800 \mathrm{~V}$ in the cold room. The gel was then stained for thirty minutes using a Coomassie Brilliant Blue stain solution (0.1\% Coomassie Brilliant Blue R-250, 50\% methanol, $10 \%$ glacial acetic acid) and a 1 hour destain using a solution containing $40 \%$ methanol and $10 \%$ glacial acetic acid. The gel was then dried using a Biorad model 583 gel dryer. 


\section{Method II: Microscale thermophoresis (MST)}

Given the difficulties of assessing tRNA binding by LeuRS-F/I via EMSA, microscale thermophoresis (MST) was employed as an alternative means of gauging these interactions. However, this methodology was deemed impossible due to the intrinsic fluorescence of both LeuRS-F and LeuRS-I decreasing following the titration of the proteins with higher concentrations of tRNA. Therefore, a secondary method detecting fluorescence quenching of tryptophan residues on the proteins following the potential binding of tRNA ${ }^{\text {Leu }}$-UAG substrate was performed utilizing the Monolith NT.Label Free (NanoTemper Technologies) instrument. Binding reactions were assembled using $15 \mathrm{mM} \mathrm{MgCl}_{2}, 10 \mu \mathrm{M} \mathrm{ZnSO}_{4}, 25 \mathrm{mM} \mathrm{NaCl}, 2 \mathrm{mM}$ DTT, $12.5 \%$ glycerol, $60 \mathrm{mM}$ MES (pH 6.5), 0.1\% pluronic F-127, in vitro transcribed tRNA ${ }^{\mathrm{Leu}}-\mathrm{UAG}$, and 500nM LeuRS-F or LeuRS-I. A set of three varied concentrations of folded tRNA ${ }^{\text {Leu }}$-UAG substrate was utilized in a range from $75 \mu \mathrm{M}$ to $0.59 \mu \mathrm{M}$. All binding reactions proceeded with were incubated at room temperature for at least 1 hour and were loaded into standard-treated capillaries (NanoTemper Technologies). NT analysis software provided by NanoTemper Technologies was used to aggregate the fluorescence scans generated by the instrument and to normalize the complexed molecule fraction. Three biological replicates were then introduced into KaleidaGraph (Synergy Software) and were fitted to the Hill equation to asses binding affinities of the protein and tRNA molecules. Remaining portions of binding reactions were then subjected to an SD denaturing protocol to ensure that fluorescence quenching was indeed was a result of protein-tRNA interaction and not errant precipitation of the protein sample under heightened tRNA concentrations. Binding reactions were centrifuged at 14,000 rpm for 11 minutes, with a portion of this reaction combined to equal volumes of SD buffer ( $40 \mathrm{mM}$ DTT and $4 \% \mathrm{SDS}$ ) reagents, then heated at $95^{\circ} \mathrm{C}$ for 5 
minutes. Samples were then loaded into capillaries and scanned using the Monolith NT.Label Free instrument.

\section{Phylogenetic analyses of LeuRS within the Sulfolobaceae}

As a means of assessing the prevalence of this paralogous synthetase amongst different strains of S. islandicus and other members of the family, phylogenetic analysis of 39 family members of the Sulfolobaceae was performed.

\section{Method I: Sequence gathering and phylogeny generation}

For phylogenetic analysis, the open source software known as Mega X: Molecular Evolutionary Genetics Analysis was used. ${ }^{[38]}$ Sequences with considerable homology to $S$. islandicus LeuRS-F and LeuRS-I were identified and retrieved from each family member analyzed. A variety of online databases were utilized for sequence collection, including Uniprot, NCBI, and KEGG. ${ }^{[39,40,41]}$

Because of a lack of annotation of certain sequences, the identity of LeuRS-I had to be determined manually by assessing the substitutions of critical amino acids in the CP1 domain that are central for the editing capacity of these proteins, namely the conserved aspartic acid at position 315 which is substituted with histidine in LeuRS-I. ${ }^{[15,16]}$ The compiled sequences were aligned within Mega X and, using the nearest neighbor analysis method with a 1000 iteration bootstrap phylogeny test and Poisson modelling, the phylogeny was generated. Poisson modelling is a regression analysis that models response variables and indicates which explanatory variables are statistically significant. ${ }^{[42]}$ Condensed tree formatting was employed at a $75 \%$ cutoff score for

producing the phylogeny. This cutoff score reflects only sequences that the program assesses as having a $75 \%$ confidence of being at a given position. 


\section{Generation of a LeuRS-I antibody}

For tracking the in vivo expression of LeuRS-I, the amino acid sequence corresponding to the C-terminal fragment, residues 789 - 934, of LeuRS-I was cloned into pET14b(+) (Novagen) generating a clone fused to an N-terminal His-tag. This clone was transformed and overexpressed in E. coli BL21(DE3) (New England Biolabs) and subsequently purified for the production of polyclonal antibodies (Pierce Custom Antibodies). This particular sequences was chosen to decrease the chances of cross reactivity with LeuRS-F.

\section{Method I: Transformation and overexpression}

Ten milliliters of an E. coli BL21(DE3) starter culture harboring a plasmid coding for the C-terminal domain of LeuRS-I [His6-LeuRS-I (789-934)] was inoculated into 1L of lysogeny broth (LB) and allowed to grow at $37^{\circ} \mathrm{C}$ with aeration at $250 \mathrm{rpm}$ until an $\mathrm{OD}_{600}$ of 0.433 was reached. At this time, protein expression was induced with $400 \mu \mathrm{M}$ isopropyl $\beta$-D-1-thiogalactopyranoside (IPTG) ${ }^{[43]}$ Induced cultures were shifted to room temperature and allowed to grow overnight with aeration $125 \mathrm{rpm}$. Cells were harvested on the subsequent day via centrifugation at $4250 \mathrm{rpm}$ for 45 minutes at $4^{\circ} \mathrm{C}$. The cell pellet was resuspended in $20 \mathrm{~mL}$ of lysis buffer $(300 \mathrm{mM} \mathrm{NaCl}, 50$ $\mathrm{mM} \mathrm{Na}_{2} \mathrm{HPO}_{4}, 10 \mathrm{mM}$ imidazole, $10 \%$ glycerol, $\mathrm{pH}$ 8.0) and froze at $-20^{\circ} \mathrm{C}$. Cells were thawed and doped with $1 \mathrm{mM}$ Phenylmethylsulfonyl fluoride, $1 \mathrm{mg} / \mathrm{mL}$ lysozyme and 1 Pierce protease inhibitor pill, then was subsequently lysed via sonication using a Fisher Scientific Sonic Dismembrator. Sonication parameters included $30 \%$ power for a total of 4 minutes of sonication time in 30 second intervals of on / off states to release the expressed protein into the soluble fraction. Lysates were cleared via centrifugation at 14,000 x g for 30 minutes. 


\section{Method II: Affinity purification of overexpressed protein}

For selective purification of the overexpressed protein, an agarose NTA-Ni ${ }^{2+}$ column with a $2 \mathrm{~mL}$ bed volume was utilized to capture the His-tagged LeuRS-I (789-934) fragment from the lysate. To begin, the soluble fraction of the cleared and centrifuged lysate was loaded on the nickel column and allowed to flow through. The column was subsequently rinsed as follows: $20 \mathrm{~mL}$ lysis buffer, $10 \mathrm{~mL}$ imidazole wash buffer $1\left(10 \mathrm{mM}\right.$ imidazole, $300 \mathrm{mM} \mathrm{NaCl}, 50 \mathrm{mM} \mathrm{Na} 2 \mathrm{HPO}_{4}, 10 \%$ glycerol, $\mathrm{pH}$ 8.0), $10 \mathrm{~mL}$ imidazole wash buffer 2 (15 mM imidazole, maintaining same salt concentrations), and imidazole wash buffer 3 (20 mM imidazole, maintaining same salt concentrations), and was subsequently eluted in $10 \mathrm{~mL}$ of elution buffer ( $250 \mathrm{mM}$ imidazole, 300 $\mathrm{mM} \mathrm{NaCl}, 50 \mathrm{mM} \mathrm{Na}_{2} \mathrm{HPO}_{4}, 10 \%$ glycerol, $\mathrm{pH} 8.0$ ). A $15 \%$ SDS-PAGE was used to track the affinity purification of this protein. The C-terminal fragment is $19.2 \mathrm{kD}$ in size. After confirmation of the presence His6-LeuRS-I (789-934) post purification, the protein was dialyzed into phosphate buffered saline (PBS; $137 \mathrm{mM} \mathrm{NaCl}, 2.58 \mathrm{mM} \mathrm{KCl}, 10.14 \mathrm{mM} \mathrm{Na}_{2} \mathrm{HPO}_{4}, 1.76 \mathrm{mM} \mathrm{KH}_{2} \mathrm{PO}_{4}, \mathrm{pH}$ 7.4), supplemented with $1 \mathrm{mM}$ dithiothreitol (DTT), $2 \mathrm{mM}$ EDTA, and 10\% glycerol. Upon completion of dialysis, protein concentrations were determined using a BioRad Bradford assay using the manufacturer protocol.

\section{Method III: Thrombin digestion}

The purified protein contains a thrombin cleavage site between the His-tag and the desired protein sequence. Therefore, to generate a protein more closely resembling the wild type LeuRSI C-terminal domain fragment, the fusion protein was subsequently processed with 2 units of biotinylated thrombin to remove the His-tag. Cleavage kinetics were followed via 15\% SDSPAGE over a 16-hour time course. Biotinylated thrombin was removed from solution by adding $200 \mu \mathrm{L}$ of streptavidin coated agarose beads (Novagen) and capturing the bead-streptavidin- 
biotinylated thrombin complex using filter-containing microfuge spin columns (Novagen). Liberated His-tag and uncleaved His-LeuRS-I (789-934) was captured using agarose Ni-NTA ${ }^{2+}$ resin, leaving only the desired C-terminal fragment of LeuRS-I as determine via PAGE analysis.

The collected protein was dialyzed using protein storage buffer $(15 \mathrm{mM} \mathrm{NaCl}, 2.58 \mathrm{mM}$ $\left.\mathrm{KCl}, 10.14 \mathrm{mM} \mathrm{Na}_{2} \mathrm{HPO}_{4}, 1.76 \mathrm{mM} \mathrm{KH} \mathrm{PO}_{4}\right), \mathrm{pH}$ 7.4, supplemented with $1 \mathrm{mM}$ DTT, $1 \mathrm{mM}$ EDTA, and $10 \%$ glycerol and was further purified via anion exchange chromatography using diethylaminoethyl (DEAE) resin. A step gradient was used to elute bound LeuRS-I (789-934) from this column in a range from $75 \mathrm{mM} \mathrm{NaCl}$ to $1 \mathrm{M} \mathrm{NaCl}$. Subsequent PAGE analysis determined that the LeuRS-I (789-934) eluted from the DEAE column at a salt concentration of $75 \mathrm{mM}$ sodium chloride. The digested and purified C-terminal domain of LeuRS-I was pooled and dialyzed against PBS, 5\% glycerol, $1 \mathrm{mM}$ DTT, and $2 \mathrm{mM}$ EDTA, $\mathrm{pH} 7.5$. The protein was used to immunize rabbits for the production of polyclonal antibodies specific to this domain (Pierce Custom Antibodies, 90-day rabbit protocol, 2 rabbits).

\section{Generation of a S. islandicus genomic C-terminal HA-tagged LeuRS-I (I-Ct-HA)}

The construction of a tagged genomic version of LeuRS-I (I-Ct-HA) is of importance for a variety of biological assays and can be used in conjunction with a previously generated Cterminal FLAG-tagged LeuRS-F strain (F-Ct-FLAG).

\section{Method I: Plasmid construction}

Through recombinant DNA cloning, a plasmid was constructed for introduction of an Hemagglutinin-tag (HA-tag) fused to the C-terminus of LeuRS-I. This HA-tag consists of 9 amino acids (YPYDVPDYA) and can be readily detected with commercially available antibodies. Vector pRJW08, an E. coli $-S$. islandicus suicide vector, can be actively propagated in $E$. coli but not $S$. islandicus. ${ }^{[11]}$ A 1.68 kilobase pair (kbp) fragment was synthesized (IDT, referred to as gBlock), 
consisting of a $730 \mathrm{bp}$ 3' LeuRS-I gene fragment (target gene; $\mathrm{Tg}$ ) fused to the HA-tag sequence and a 868 bp downstream (D1) fragment. The gBlock fragment was diluted to $100 \mu \mathrm{M}$ with a 10 $\mathrm{mM}$ Tris-HCl buffer, $\mathrm{pH}$ 8.8. Ten fmol of amplified, Dpn1 digested, and cleaned pRJW08 vector was combined with 30 fmol of pre-diluted gBlock fragment, 1X HiFi Gibson master mix (NEB) and taken to a final volume of $10 \mu \mathrm{L}$ with $\mathrm{ddH}_{2} \mathrm{O}$. The reaction was run on a BioRad $\mathrm{C} 1000$ Touch thermocycler for 30 minutes at $50^{\circ}$ C. E. coli Endura chemically competent cells (Lucigen) were transformed with $2 \mu \mathrm{L}$ of this reaction according to the manufacturer's recommendations in the hopes of isolating successfully constructed recombinant plasmids. Endura cells were used as they possess a genotype that appears capable of maintaining toxic DNA products. Transformations were plated on LB agar supplemented with $100 \mu \mathrm{g} / \mathrm{mL}$ carbenicillin and incubated overnight at both $37^{\circ} \mathrm{C}$ and room temperature. Colony PCR was used to screen resulting colonies for successfully constructed recombinant clones. Prior to using colonies as template for these PCR reactions, they were patch-struck onto fresh $100 \mu \mathrm{g} / \mathrm{mL}$ LB-carbenicillin plates. Remaining portions of colonies were resuspended in $10 \mu \mathrm{L}$ of $\mathrm{ddH}_{2} \mathrm{O}$ generating PCR templates. Twenty microliter reactions contained $5 \mu \mathrm{L}$ of resuspended colony template, $0.5 \mu \mathrm{M}$ primer M9, $0.5 \mu \mathrm{M}$ primer REV99, and 1X GoTaq mastermix (NEB). Thermocycler settings included an initial melting temperature of $95^{\circ} \mathrm{C}$ for 2 minutes, melting temperature of $95^{\circ} \mathrm{C}$ for 30 seconds, annealing temperature of $55^{\circ} \mathrm{C}$ for 35 seconds, extension temperature of $72^{\circ} \mathrm{C}$ for 2 minutes and 20 seconds for 39 cycles, a final extension at $72^{\circ} \mathrm{C}$ for 3 minutes followed by a hold at $4{ }^{\circ} \mathrm{C}$. Samples were loaded into a $1 \%$ (wt/vol) agarose LE gel in $1 \mathrm{X}$ TAE and were electrophoresed for 40 minutes. DNA was stained using ethidium bromide (EtBr, $1 \mu \mathrm{g} / \mathrm{mL}$ in $\mathrm{ddH}_{2} \mathrm{O}$ bath) and subsequently imaged on a UVP GelDoc-IT system. 
Colony patches corresponding to positive screens were subsequently grown overnight at $37^{\circ} \mathrm{C}$ with aeration in $5 \mathrm{~mL} \mathrm{LB}$ supplemented with $100 \mu \mathrm{g} / \mathrm{mL}$ carbenicillin. Three to four milliliters of this overnight culture was used to isolate plasmid DNA using an IBI miniprep kit according to the manufacturers instructions. DNA was eluted in $40 \mu \mathrm{L}$ of pre-warmed elution buffer. Samples were given identities based on the condition at which the colony was initially grown (RT and IC for room temperature and incubator), as well as a number descriptor for the colony identity. Samples included RT1, RT2, IC1, and IC2. Isolated plasmids were PCR screened for the gBlock fragment as previously described. IC1 was sequence verified via Sanger sequencing at the University of Illinois, Urbana-Champaign (UIUC) Core DNA facility and was subsequently carried forward in additional cloning steps.

pRJW08-gBlock(+) was double digested in $20 \mu \mathrm{L}$ reactions using the endonucleases SalIHF (NEB, 20 units) and XmaI (NEB, 10 units) in $1 \mathrm{X}$ CutSmart buffer (NEB). Reactions were incubated at $37^{\circ} \mathrm{C}$ for 4 hours and were subsequently purified using an IBI PCR cleanup kit following the manufacturer's protocol. Digested DNA was eluted in a final volume of $30 \mu \mathrm{L}$ using prewarmed elution buffer (10mM Tris- $\mathrm{HCl} \mathrm{pH} 8.8)$.

To complete the plasmid needed to insert the HA-tag onto the C-terminus of LeuRS-I, an identical downstream fragment as D1 (D2) containing terminal SalI and XmaI restriction sites was required. This DNA fragment makes the suicide vector proficient for recombination with the $S$. islandicus genome. Previously isolated gDNA from S. islandicus M16.4 strain was used as the template for amplification of this D2 fragment. PCR reactions contained 1X Q5 Buffer (NEB), 1X Q5 enhancer (NEB), $0.2 \mathrm{mM}$ dNTP mix, $50 \mathrm{ng}$ S. islandicus gDNA, $0.5 \mu \mathrm{M}$ primer M57, $0.5 \mu \mathrm{M}$ primer M58, and 1 U Q5 DNA Polymerase (NEB). Reactions were run on a minicycler program including an initial melt at $98^{\circ} \mathrm{C}$ for 2 minutes followed by 29 cycles of the following: $98^{\circ} \mathrm{C}$ for 
30 seconds, $55^{\circ} \mathrm{C}$ for 30 seconds, $72^{\circ} \mathrm{C}$ for 1 minute. A final extension at $72^{\circ} \mathrm{C}$ for 5 minutes was subsequently followed by an infinite incubation at $4^{\circ} \mathrm{C}$. After PCR amplification, product quantity and purity were qualitatively assessed using $1 \%$ (wt/vol) agarose gel electrophoresis. Afterwards, amplified D2 fragment was purified using an IBI PCR cleanup kit, again, following the provided manufacturer's protocol. DNA was eluted in $20 \mu \mathrm{L}$ of pre-warmed elution buffer and double digested at $37^{\circ} \mathrm{C}$ overnight using 20 units of SalI and 10 units of $\mathrm{XmaI}$.

Double digested pRJW08-gBlock(+) (10 fmol) and D2 (40 fmol) were ligated in $10 \mu \mathrm{L}$ reactions containing the indicated amounts of DNA, 1X T4 DNA Ligase buffer, and 400 U of T4 DNA Ligase. Ligations were incubated at room temperature for 30 minutes followed by a $15 \mathrm{~min}$ incubation at $65^{\circ} \mathrm{C}$ to heat kill the ligase. E. coli $\underline{\text { Stop }} \underline{\text { Unwanted }}$ Rearrangement Event competent cells (SURE2, Agilent) were transformed with $(2 \mu \mathrm{L})$ of this ligation reaction following standard transformation protocols. Transformed cells were selected on LB agar plates supplemented with $100 \mu \mathrm{g} / \mathrm{mL}$ carbenicillin at $37^{\circ} \mathrm{C}$ overnight. Colony PCR was executed on resulting colonies using GoTaq master mix and primer pair M87 and FOR88. Successfully screened colonies were grown and miniprepped, and one of the acquired recombinant clones was sequence verified at UIUC's Core DNA facility.

\section{Method II: Transformation of pRJW08-gBlock/D2(+) into wild type S. islandicus}

Following construction of plasmid pRJW08-gBlock/D2(+) (HA-I), wild type S. islandicus M.16.4 (RJW04, $\Delta$ pyrEF $\Delta \operatorname{lacS} \Delta \arg D)^{[11]}$ was prepared for transformation. Wild type cells were recouped from freezer stocks as previously described and subsequently passaged into $20 \mathrm{~mL}$ DT/AU medium. Cells were grown at $75^{\circ} \mathrm{C}$ to an $\mathrm{OD}_{600}$ of 0.350 and were harvested via centrifugation at $8,000 \times \mathrm{g}$ for 15 minutes at $4{ }^{\circ} \mathrm{C}$. Following centrifugation, the cell pellet was rinsed with $15 \mathrm{~mL}$ of $20 \mathrm{mM}$ sucrose thrice, decanting the sucrose supernatant after each 
subsequent centrifugation step. Cells were resuspended in a final volume of $1 \mathrm{~mL} 20 \mathrm{mM}$ sucrose and were carried forward for transformation.

The HA-I vector required linearization prior to its introduction into the genome of wild type $S$. islandicus. Roughly $8.5 \mu \mathrm{g}$ of recuperated HA-I was digested using 20 units of XmaI. The digestion was incubated at $37^{\circ} \mathrm{C}$ overnight and was subsequently purified using a Monarch PCR cleanup kit (NEB). The standard protocol provided by the manufacturer was utilized, eluting in a final volume of $20 \mu \mathrm{L}$ with pre-warmed elution buffer (10 $\mathrm{mM}$ Tris- $\mathrm{HCl} \mathrm{pH}$ 8.8). Sample concentration and purity was determined using a NanoDrop One and the preloaded dsDNA protocol.

Aliquots of $S$. islandicus $(50 \mu \mathrm{L})$ in sucrose were combined with $1 \mu \mathrm{g}, 1.5 \mu \mathrm{g}$, and $2.0 \mu \mathrm{g}$ total linearized HA-I for transformations. Cells and DNA were incubated on ice for 30 minutes. Following incubation, the cells and DNA mix were transferred to electroporation cuvettes $(0.1 \mathrm{~cm}$ gap size, GenePulser Xcell) and were electroporated using a Gene Pulser (BioRad) set to $1.2 \mathrm{kV}$, $25 \mu \mathrm{F}$ and $600 \Omega$. Cells were removed from the electroporation cuvette and incubated in $950 \mu \mathrm{L}$ of $75^{\circ} \mathrm{C}$ recovery medium $(1 \% \mathrm{w} / \mathrm{v}$ sucrose, $20 \mathrm{mM}$ beta-alanine, $1.5 \mathrm{mM}$ malic acid, $10 \mathrm{mM}$ $\mathrm{MgSO}_{4}, \mathrm{pH} 4.5$ ) and were allowed to incubate at $75^{\circ} \mathrm{C}$ for 30 minutes. $5 \mathrm{~mL}$ of freshly autoclaved and sterilized $0.8 \%(\mathrm{wt} / \mathrm{vol}$ ) Gelzan (Sigma-Aldrich) solution and $5 \mathrm{~mL}$ of $2 \mathrm{X}$ Basal 4 DT media (0.000002\% trace metal, $0.3 \% \mathrm{~K}_{2} \mathrm{SO}_{4}, 0.05 \% \mathrm{NaH}_{2} \mathrm{PO}_{4}, 0.1405 \% \mathrm{MgSO}_{4}, 0.048 \% \mathrm{CaCl}_{2} \cdot 2 \mathrm{H}_{2} \mathrm{O}$, 0.1\% EZMix N-Z-Amine A, 0.1\% Dextrin, wt/vol) $\mathrm{pH} 2.6$ was aliquoted for each transformation and placed at $75^{\circ} \mathrm{C}$ for 30 minutes. After incubation had concluded, the cells were removed from the incubator along with the Gelzan and 2X Basal 4 DT solutions, all of which were combined in the laminar flow hood and poured over DT plates that had been thoroughly pre-heated at $75^{\circ} \mathrm{C}$. 
Overlays containing transformed cells were allowed to solidify and were subsequently incubated at $75^{\circ} \mathrm{C}$ for 2 weeks.

Following the extended incubation, embedded colonies were noted on both the $1 \mu \mathrm{g}$ and $1.5 \mu \mathrm{g}$ transformation plates and were harvested via pipetting and transferred into $2 \mathrm{~mL}$ of DT media. Cells were allowed to grow until cloudy and were stained with $2 \mu \mathrm{M}$ 5-bromo-4-chloro-3indolyl- $\beta$-D-galactopyranoside (X-gal) resuspended in DMSO. The presence of blue staining indicates a cell with the HA-I vector integrated within its genome. Positive cells were transferred to $20 \mathrm{~mL}$ of DT media and grown in the $75^{\circ} \mathrm{C}$ incubator until noticeably cloudy. After reaching an $\mathrm{OD}_{600}$ of roughly 0.400 , the cells were harvested via centrifugation under the previously mentioned parameters and were resuspended in $1 \mathrm{~mL}$ of sucrose for colony purification. $30 \mu \mathrm{L}$ of the sucrose resuspended cells were then added to a 96-well plate and 10-fold serial-diluted into $20 \mathrm{mM}$ sucrose. $200 \mu \mathrm{L}$ of the $10^{-4}, 10^{-5}$, and $10^{-6}$ dilutions were plated onto fresh DT plates and allowed to dry under flame. Plates were double bagged and placed at $75^{\circ} \mathrm{C}$ for one week after which large numbers of colonies were observed. Purified colonies were harvested as previously noted and grown in $20 \mathrm{~mL}$ of DT medium.

Removal of unnecessary plasmid-borne components still present within the genome of these transformant wild type cells was required. Counterselection was achieved by first transferring $30 \mu \mathrm{L}$ of each transformant to a 96 -well plate and ten-fold serially diluting to the $10^{-6}$ with $270 \mu \mathrm{L}$ of $20 \mathrm{mM}$ sucrose as diluent. $200 \mu \mathrm{L}$ of the $10^{-4}, 10^{-5}$, and $10^{-6}$ dilutions were used as inoculants into overlays containing $0.8 \%$ gelzan and $2 \mathrm{X}$ basal 4 counterselection medium $\left(0.000002 \%\right.$ trace metal, $0.3 \% \mathrm{~K}_{2} \mathrm{SO}_{4}, 0.05 \% \mathrm{NaH}_{2} \mathrm{PO}_{4}, 0.1405 \% \mathrm{MgSO}_{4}, 0.048 \% \mathrm{CaCl}_{2} \cdot 2 \mathrm{H}_{2} \mathrm{O}$, 0.1\% EZMix N-Z-Amine A, 0.1\% dextrin, $50 \mu \mathrm{g} / \mathrm{mL}$ 5-Fluoroorotic acid (5-FOA), $20 \mu \mathrm{g} / \mathrm{mL}$ uracil, and $1 \mathrm{mg} / \mathrm{mL}$ agmatine, wt/vol), Overlays were mixed and poured over DT/AU 
counterselection plates consisting of the same media. Plates were double-bagged and placed at $75^{\circ} \mathrm{C}$ and allowed to grow for approximately one week, after which colonies were harvested and grown in standard DT/AU medium. Screening of the strains for the integrated and LeuRS-I fused HA-tag was achieved via PCR using primer pair M97 and LRSI 1724. The integrity of all regions of the chromosome involved in the recombination, including the integrated HA-tag, were sequence confirmed at UIUC's Core DNA facilities.

\section{Growth curve of $S$. islandicus LeuRS-I-Ct-HA strain}

To assess the growth phenotype of the newly generated HA-I strain, a growth curve utilizing triplicate growth flasks of both the HA-I and S. islandicus wild type strain at the stressful temperature of $60^{\circ} \mathrm{C}$ was performed. Cells were inoculated into DT/AU medium, $\mathrm{pH} 3.25$ from freezer stocks using the previously mentioned protocol and were subsequently transferred to cell culturing flasks containing $20 \mathrm{~mL}$ DT/AU media, $\mathrm{pH} 3.25$. Passage 2 cells and were harvested at an $\mathrm{OD}_{600 \mathrm{~nm}}$ of 0.290 and 0.235 for $S$. islandicus wild type and the HA-I strain, respectively, and rinsed with sucrose as previously described. Prepped cells were inoculated in triplicate into $50 \mathrm{~mL}$ of DT/AU medium, pH 3.25 and placed in an incubator shaker set to $60^{\circ} \mathrm{C}$ and $250 \mathrm{rpm}$. Flasks were tracked for $\mathrm{OD}_{600 \mathrm{~nm}}$ over the course of 9 days at the same time on each day.

\section{Expression profiling of the HA-I strain using Western blots}

To determine the relative expression of LeuRS-I in vivo and whether this expression is modulated by the abiotic stress of low growth temperatures, HA-I and FLAG-F strains of $S$. islandicus were utilized in a comparative Western blot.

\section{Method I: Western blot analysis of fusion protein expression}

Semi-thawed freezer stocks (100 $\mu$ L) of both HA-I (strain \#86) and FLAG-F (strain \#19) strains were used to inoculate $2 \mathrm{~mL}$ of DT/AU medium and grown at $75^{\circ} \mathrm{C}$. After visible cell 
growth, $1 \mathrm{~mL}$ of each starter culture was passaged into $20 \mathrm{~mL}$ of DT/AU medium at incubated at $75^{\circ} \mathrm{C}$. Cells were grown to approximate $\mathrm{OD}_{600 \mathrm{~s}}$ of 0.350 and were harvested via centrifugation and washed as described above using $20 \mathrm{mM}$ sucrose. $\mathrm{OD}_{600}$ of the final sucrose-resuspended cells were taken. Six flasks each of FLAG-F and HA-I were inoculated to initial OD $_{600}$ of 0.008 using $150 \mathrm{~mL}$ of DT/AU medium. Three of each set were placed at $75^{\circ} \mathrm{C}$ while the other three were placed at $60^{\circ} \mathrm{C}$ with aeration $(250 \mathrm{rpm})$. Samples were monitored and harvested via centrifugation at lag, log, and early stationary phases $\left(\mathrm{OD}_{600 \mathrm{~s}}\right.$ of $0.15-0.18,0.25-0.35$, and $0.42-0.55$, respectively). A single culture for strain \#86 grown at $60^{\circ} \mathrm{C}$ was noted as losing volume.

Five milliliters of lysis buffer ( $300 \mathrm{mM} \mathrm{NaCl}, 50 \mathrm{mM} \mathrm{Na}_{2} \mathrm{HPO}_{4}, 10 \mathrm{mM}$ imidazole, $10 \%$ glycerol, $\mathrm{pH}$ 8.0) was added to harvested cell pellets. The cellular resuspensions were supplemented with a single protease inhibitor cocktail pill (Pierce) and PMSF at a final concentration of $1 \mathrm{mM}$. The culture that lost significant amounts of volume was resuspended in only $2.5 \mathrm{~mL}$ of this lysis buffer cocktail as a means of standardizing the samples. Samples were incubated on ice for $30 \mathrm{~min}$ and were then lysed using a sonic dismembrator at $30 \%$ power with 4 minutes of processing time with cycles of 30 seconds on and 30 seconds off. Tubes containing the lysed milieu were then placed on ice and spiked with Triton X-100 (Biorad) to a final concentration of $0.2 \%$ (vol/vol). These samples were incubated on ice and were gently mixed using a benchtop shaker (SHKE2000, ThermoFisher) set to $75 \mathrm{rpm}$. Following a 2-hour incubation on ice, cells were centrifuged at $8,000 \mathrm{x}$ g for 40 minutes at $4^{\circ} \mathrm{C}$ and the soluble supernatants were collected and transferred to fresh $15 \mathrm{~mL}$ falcon tubes.

Protein was quantified using a modified Bradford assay (BioRad) ${ }^{[44]}$ Due to the presence of Triton X-100 within cleared lysates and the incompatibility of the BioRad reagent to this compound, beta-cyclodextrin was added to all samples at a final concentration of $1.76 \mathrm{mM} .^{[45]}$ 
Beta-cyclodextrin acts as a molecular sponge for detergents in solution, binding to detergents such as SDS and Triton X-100 in a 1:1 molar ratio. This chemical additive aids in reducing the interference induced by detergents such as Triton X-100 when measuring absorbances on a spectrophotometer during Bradford assays. Approximate protein concentrations were gathered and normalized. A total of $1.5 \mu \mathrm{g}$ of total protein for each sample was analyzed via $8 \%$ SDS-PAGE. Separated proteins were transferred to polyvinylidene difluoride (PVDF) membrane and blocked overnight at $4{ }^{\circ} \mathrm{C}$ in Tris-buffered saline (TBS) supplemented with bovine serum albumin (TBSBSA, $50 \mathrm{mM}$ Tris- $\mathrm{HCl}$, $\mathrm{pH} 7.5,150 \mathrm{mM} \mathrm{NaCl}, 1 \% \mathrm{BSA}$ ). Target proteins were probed with either monoclonal rat anti-HA primary antibody (1:2000, Sigma, Cat\# 11867423001) or monoclonal mouse anti-FLAG primary antibody (1:2000, Sigma, Cat\# F3165) resuspended in TBS-BSA and rinse steps utilized TBS supplemented with $0.2 \%$ Tween-20 (TBS-T, BioRad). Horse radish peroxidase (HRP) conjugated goat anti-mouse and goat anti-rat secondary antibodies (KPL) at a 1:10,000 dilution in TBS-BSA, ECL chemiluminescent substrate (West Pico Plus, Thermo Fisher), X-ray film (Research Products International, Cat\# 248304) and an automatic X-ray film developer were used to develop blots. Densitometry analysis of HA-I protein was performed using FIJI (Fiji is just ImageJ). ${ }^{[46]}$ Areas surrounding the bands present on the blot were standardized in size amongst all data sampling points, with histograms generated for each subsequent sample band. Histogram intensity plots were then measured by setting a cutoff at the rise and fall of each end of the histogram. A background subtraction was taken by sampling a blank portion of the blot and subtracting the blank's intensity from each of the densitometric readings gathered for each sample. 


\section{CHAPTER III: RESULTS AND DISCUSSION}

\section{Development of a phylogenetic tree comparing LeuRS-F and LeuRS-I in the Sulfolobaceae}

As a means of assessing the prevalence of this paralogous synthetase amongst different strains of $S$. islandicus and other members of the family, phylogenetic analysis of 39 family members of the Sulfolobaceae was performed. A preliminary investigation was tasked as a means of determining whether this duplication event is a localized aberration shared between only closely related species and strains or if this genetic event is widespread and maintained beyond the genus. Genomic comparative analysis has shown that this duplication event of the LeuRS gene is conserved across all family members of the Sulfolobaceae, including those of the genus acidianus and metallosphaera (Figure 2). This duplication's omnipresence amongst Sulfolobaceae family members provides a strong indication of importance as indicated by its maintenance across evolutionary timescales in these organisms. Upon analysis, there is a clear and distinct evolutionary trajectory that each of these synthetases are following when comparing the sequences derived from these different species. Clustering of LeuRS-F and LeuRS-I into specific groups is apparent, and the trend of demarcation of the two enzymes is total. The clustering of similar species such as the different strains of $S$. islandicus is also ostensible, showing a closer relation to one another within each of these groupings as compared to different species such as Acidianus or Solfataricus. Interestingly, the positions of LeuRS-I and LeuRS-F of the Metallosphaera species cluster differently, with branching of Metallosphaera LeuRS-I along with the Acidocaldarius strains, while LeuRS-F clusters with Acidianus strains. 


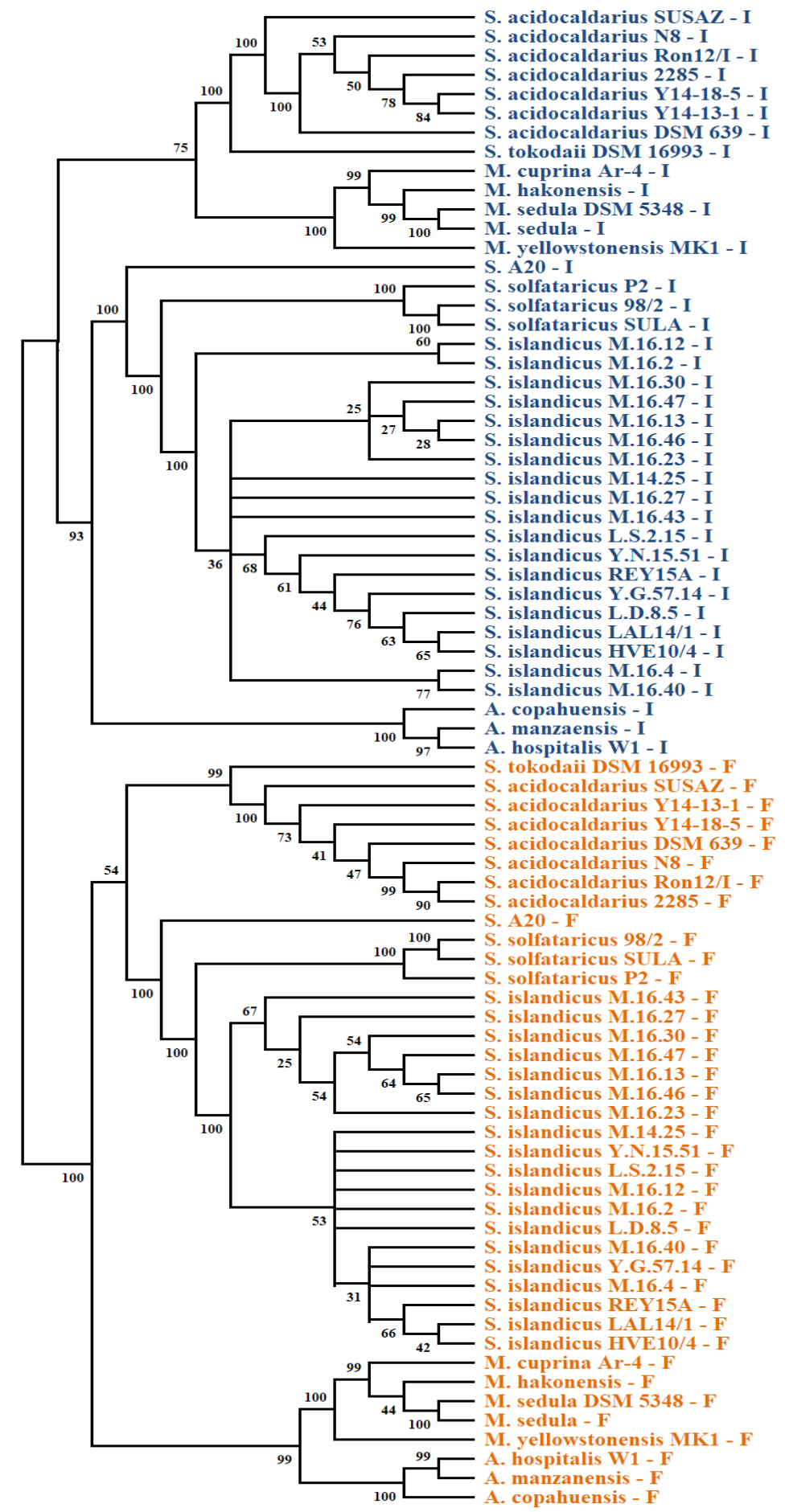

Figure 2. A phylogeny consisting of the LeuRS-I and LeuRS-F enzymes across the family Sulfolobaceae

A Mega X phylogenetic analysis of LeuRS-I and LeuRS-F across a variety of different species (39) within the family Sulfolobaceae. Bootstrap values are shown at node points relaying confidence of the strain's placement in its position. S - Sulfolobus. M - Metallosphaera. A Acidianus. Colors: Orange - LeuRS-F; Blue - LeuRS-I. 


\section{Electrophoretic mobility shift assays}

Due to the inability of LeuRS-I to aminoacylate in vitro transcribed tRNA ${ }^{\text {Leu }}$, a question of whether this enzyme is capable of binding tRNA at all has been raised. The logical assessment of this issue would be that LeuRS-I is incapable of binding tRNA ${ }^{\text {Leu }}$ and therefore cannot complete the charging of leucine onto the cognate tRNA. In an attempt to assess the ability of LeuRS-I to bind tRNA ${ }^{\text {Leu }}$ substrates, electrophoretic mobility shift assays (EMSA) were employed. The principle of EMSA is such that the interaction of both a protein and nucleic acid species can be visualized on Native-PAGE as a "shift" or a banding pattern that presents itself as a higher molecular weight than merely the protein or nucleic acid alone. ${ }^{[49]}$ This higher weight band signifies the binding interaction between the protein and nucleic acid substrate. This technique has been used to assess the binding affinities of other tRNA synthetases to their respective tRNA substrates. ${ }^{[50]}$

To begin, a TBM gel was run with 2-fold serial diluted LeuRS-I and a consistent quantity of radiolabeled in vitro transcribed tRNA ${ }^{\text {Leu }}$-UAG. Reaction conditions included a supplemental buffer consisting of $295 \mu \mathrm{M}$ MES, pH 6.7, $75 \mu \mathrm{M} \mathrm{ZnSO}_{4}$ and $20 \mu \mathrm{M} \mathrm{NaCl}$ and a 10x folding mix comprised of $10 \mu \mathrm{M}$ non-radiolabeled tRNAleu-UAG (cold UAG) and $10 \mu \mathrm{M}$ radiolabeled tRNA ${ }^{\text {Leu }}$ UAG (hot UAG), $5 \mu \mathrm{M} \mathrm{MgCl} 2,10 \mu \mathrm{M}$ MES. The TBM gel was noted as having illdefined resolution and failed to show a clear and well-defined shift representing the interaction between LeuRS-I and this tRNA substrate. However, it was clear that a minor portion of the tRNA was aggregating, represented as signal in some of the gel wells (Figure 3). The doublet near the bottom of the gel is representative of the radiolabeled tRNA. The top band of the two represents the functional tRNA substrate as deduced from its disappearance in lane one which contains the highest concentration of LeuRS-I (Figure 3). The bottom of the two bands likely represents a 
degraded tRNA ${ }^{\text {Leu }}$-UAG variant that was labeled during the kinase reaction. Alternatively, this band may represent the full-length tRNA that has adopted a different tertiary structure. As alluded to above, although no discernable shift was apparent, lane one of the TBM gel containing undiluted protein with tRNA presents itself as the most "non-mobile" sample as noted by the highest intensity of tRNA substrate at the top of the gel. Subsequent lanes consist of two-fold diluted protein.

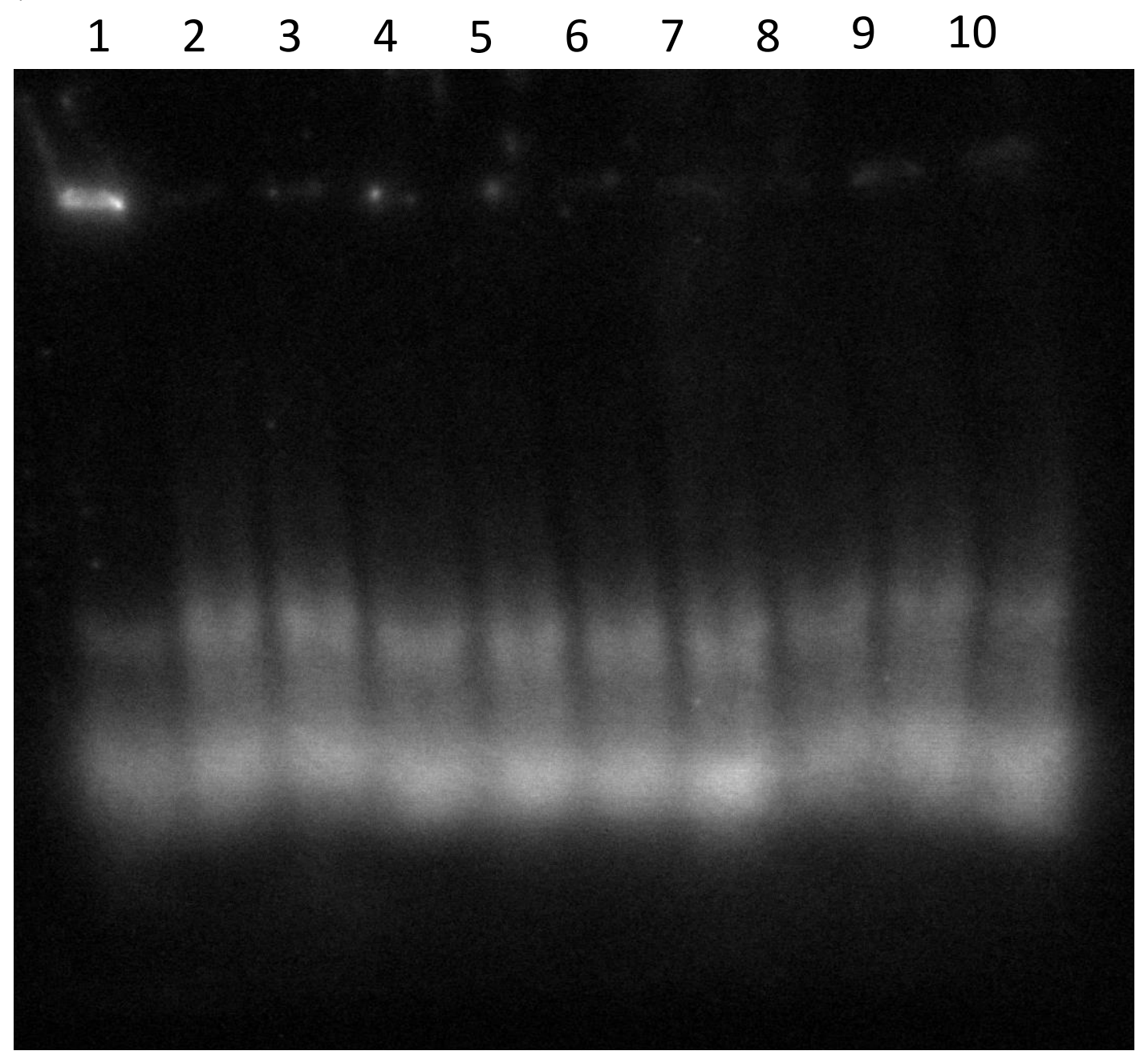

Figure 3. A 5\% TBM EMSA gel monitoring the binding of LeuRS-I and $\left[{ }^{32} \mathrm{P}\right]-\mathrm{tRNA}{ }^{\mathrm{Leu}}$ UAG.

A 5\% TBM gel comprised of 37.5:1 acrylamide:bisacrylamide run with a $0.5 x$ TBM buffer until dye front approached the bottom of the plate. Gel contains a full set of serial diluted protein from lane $1-10$. A total of $50 \mathrm{nM}$ tRNA ${ }^{\text {Leu }}-\mathrm{UAG}$ was utilized in addition to each of the protein samples. Lane 1 consists of $4.4 \mu \mathrm{M}$ final protein, lane 2 equates to $2.2 \mu \mathrm{M}$ final protein and subsequent lanes follow 2-fold dilutions. Lane 10 consists of a final protein concentration of $0.0085 \mu \mathrm{M}$. 
To further explore and optimize the EMSA system, a secondary gel buffered with a bis-tris solution and utilizing a MOPS running buffer was employed. Two separate gels of varied acrylamide:bisacrylamide ratios were made in order to modulate pore size and LeuRS-F was used knowing that this synthetase robustly charges tRNA ${ }^{\mathrm{Leu}}$ and must, therefore, bind this substrate (Figures 4 and 5). While a discernable gel shift was not apparent, radioactive signal was detected

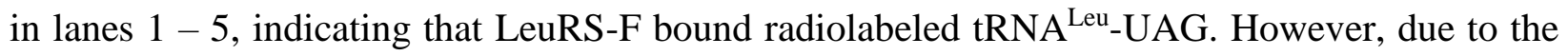
poor quality of both gels and the inability of the LeuRS-tRNA complex to migrate beyond the wells, binding affinities could not be calculated. In fact, discernable free tRNA bands were difficult to distinguish. It was also clear that neither ratio of acrylamide:bisacrylamide used to pour these gels affected the observed migration patterns.

Next, bis-tris gels were poured using a consistent acrylamide:bisacrylamide ratio (37.5:1) supplemented with either glycerol or triethylene glycol (TEG), which has been previously reported as a stabilizing factor in DNA-protein complexing. ${ }^{[36]}$ Assays used consistent quantities of $\left[{ }^{32} \mathrm{P}\right]-$

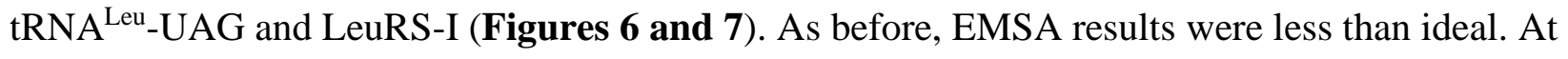
the concentration of TEG tested (20\%), this reagent was found to be unsuitable for EMSA experiments. And, while the gel substituted with 50\% glycerol was of higher quality, it was still somewhat ill-defined and fuzzy. A tRNA only control lane was omitted within this reaction set, and neither assay condition displayed a clear gel shift. However, a greater degree of streaking can be noted in the first three lanes of each gel where higher concentrations of protein are present. This streaking is interpreted to represent a LeuRS-I-tRNA ${ }^{\text {Leu }}$ interaction.

Because gel composition, pore size, and buffering components appeared to have little effect on the ability to produce a clear shift, we explored the possibility that the majority of the tRNA substrate was in an inactive conformation. To address this hypothesis, one tRNA pool was folded 
using the typical "slow cool" protocol while a second pool was put through a "snap cool" protocol where after an initial incubation at temperatures hovering around $90^{\circ} \mathrm{C}$, the tRNA was placed directly in ice. These assays also utilized 5\% bis-tris gels (37.5:1) consisting of a 50\% glycerol solution, a consistent quantity of $\left[{ }^{32} \mathrm{P}\right]-\mathrm{tRNA}{ }^{\mathrm{Leu}}-\mathrm{UAG}$, and varying amounts of LeuRS-I (Figure 8). Again, a clear shift representing a synthetase-tRNA interactions was not observed. However, as gauged by lanes $1-3$, the quality of the standard preparation of folding mix appeared to be of substantially higher quality.

Due to concerns about the quality of the $\left[{ }^{32} \mathrm{P}\right]-\mathrm{tRNA}{ }^{\mathrm{Leu}}-\mathrm{UAG}$ substrate and its advanced decay, the use of cold tRNA ${ }^{\text {Leu }}$-UAG along with an alternative staining method was explored. SYBR ${ }^{\circledR}$ Gold (Thermo Fisher Scientific) has been shown to be a versatile and highly sensitive stain for RNA and has already successfully been used in EMSA as reported by Seo and colleagues. ${ }^{[37]}$ To test its applicability within our system, varying concentrations of cold tRNA ${ }^{\text {Leu }}$ UAG were folded or left unfolded in the presence and absence of our standard EMSA supplemental buffer. The folding mixes were then separated on a 5\% TBM gel (37.5:1 acrylamide:bisacrylamide) and stained with SYBR Gold according to the manufacturer's recommendations (Figure 9). As reported, the stain is very sensitive, detecting as little as $17.6 \mathrm{ng}$ loaded (Figure 9, Lane 1) suggesting that its application in our EMSA assays may prove beneficial. In addition, this gel shows that, although a tRNA folding protocol is beneficial for decreasing alternative RNA structures adopted by UAG (Figure 9, arrows) the majority of "unfolded" tRNA exists in the correct tertiary conformation. Finally, it is clear that the addition of supplemental buffer to our tRNA folding mixes has no impact on the structure adopted by tRNA ${ }^{\text {Leu }}$-UAG alleviating suspicions of this possibility (Figure 9, compare Lanes 2 and 3 with Lanes 4 and 5). 


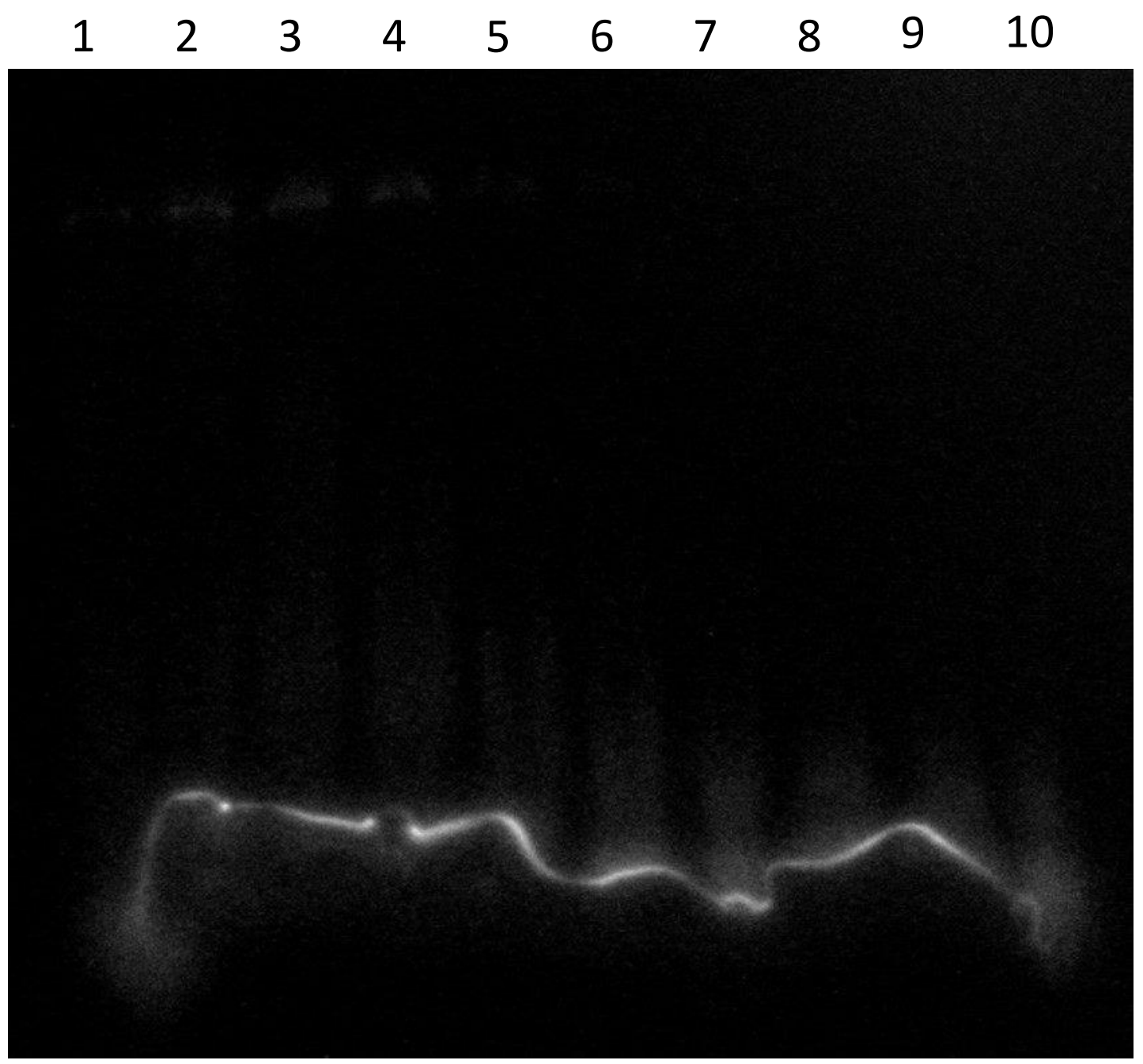

Figure 4. Acrylamide (37.5:1) pore size and its effect on the binding of LeuRS-F to $\left[{ }^{32} \mathrm{P}\right]-$

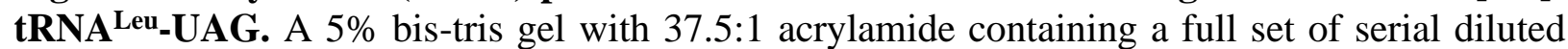
protein from lane $1-9$, with a final tRNA-only control in lane 10 . A total of $50 \mathrm{nM}$ tRNA $^{\text {Leu }}$ UAG was utilized in addition to each of the protein samples. Lane 1 consists of $1.7 \mu \mathrm{M}$ final protein, lane 2 equates to $0.85 \mu \mathrm{M}$ final protein and subsequent lanes follow 2 -fold dilutions. Lane 9 consists of a final protein concentration of $0.0066 \mu \mathrm{M}$. 
$\begin{array}{llllllllll}1 & 2 & 3 & 4 & 5 & 6 & 7 & 8 & 9 & 10\end{array}$

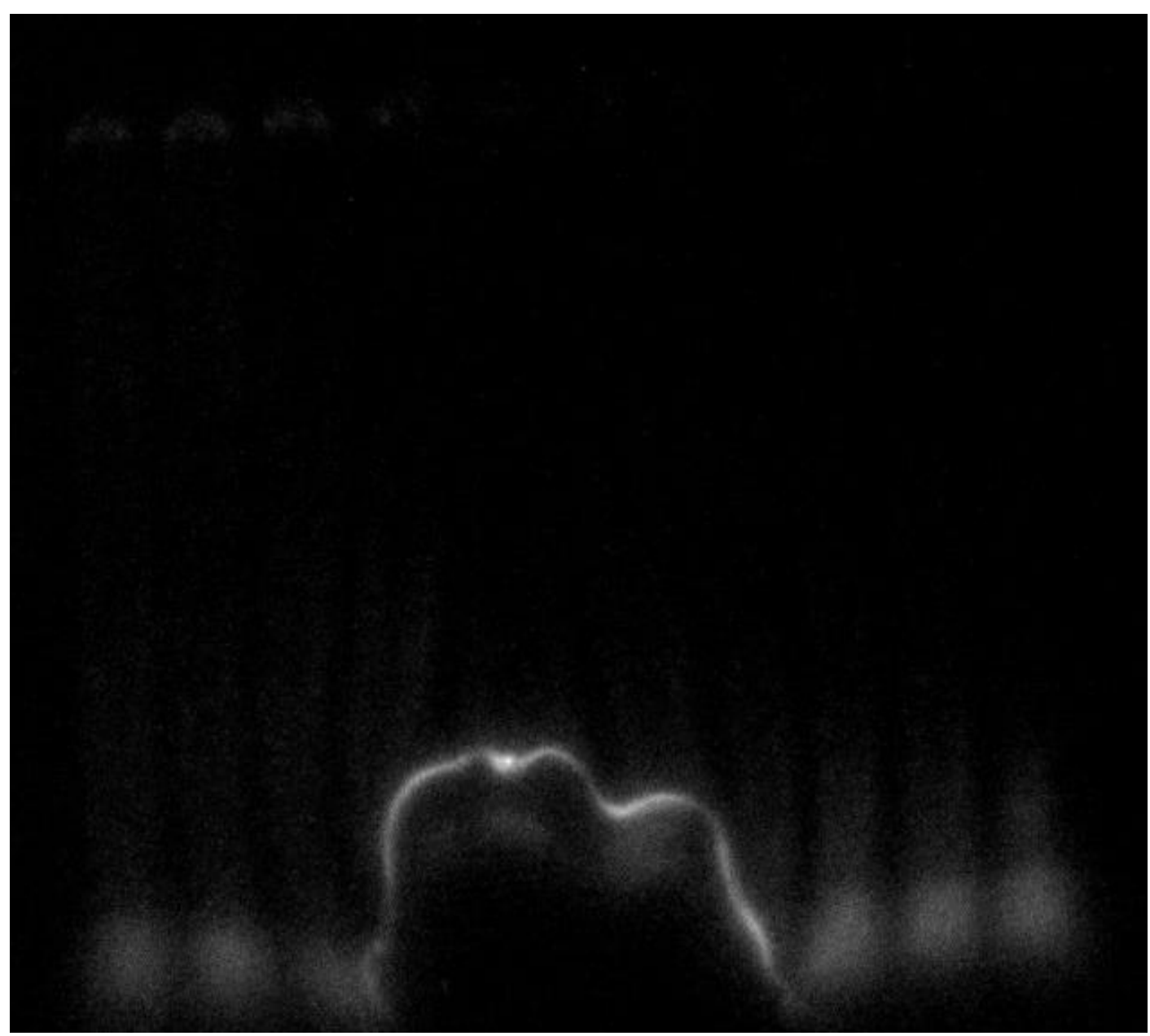

Figure 5. Acrylamide (29:1) pore size and its effect on the binding of LeuRS-F to $\left[{ }^{32} \mathrm{P}\right]$ -

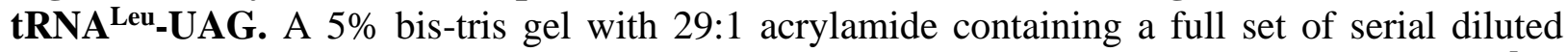
protein from lane $1-9$, with a final tRNA-only control in lane 10 . A total of $50 \mathrm{nM}$ tRNA ${ }^{\text {Leu }}$ UAG was utilized in addition to each of the protein samples. Lane 1 consists of $1.7 \mu \mathrm{M}$ final protein, lane 2 equates to $0.85 \mu \mathrm{M}$ final protein and subsequent lanes follow 2-fold dilutions. Lane 9 consists of a final protein concentration of $0.0066 \mu \mathrm{M}$. 


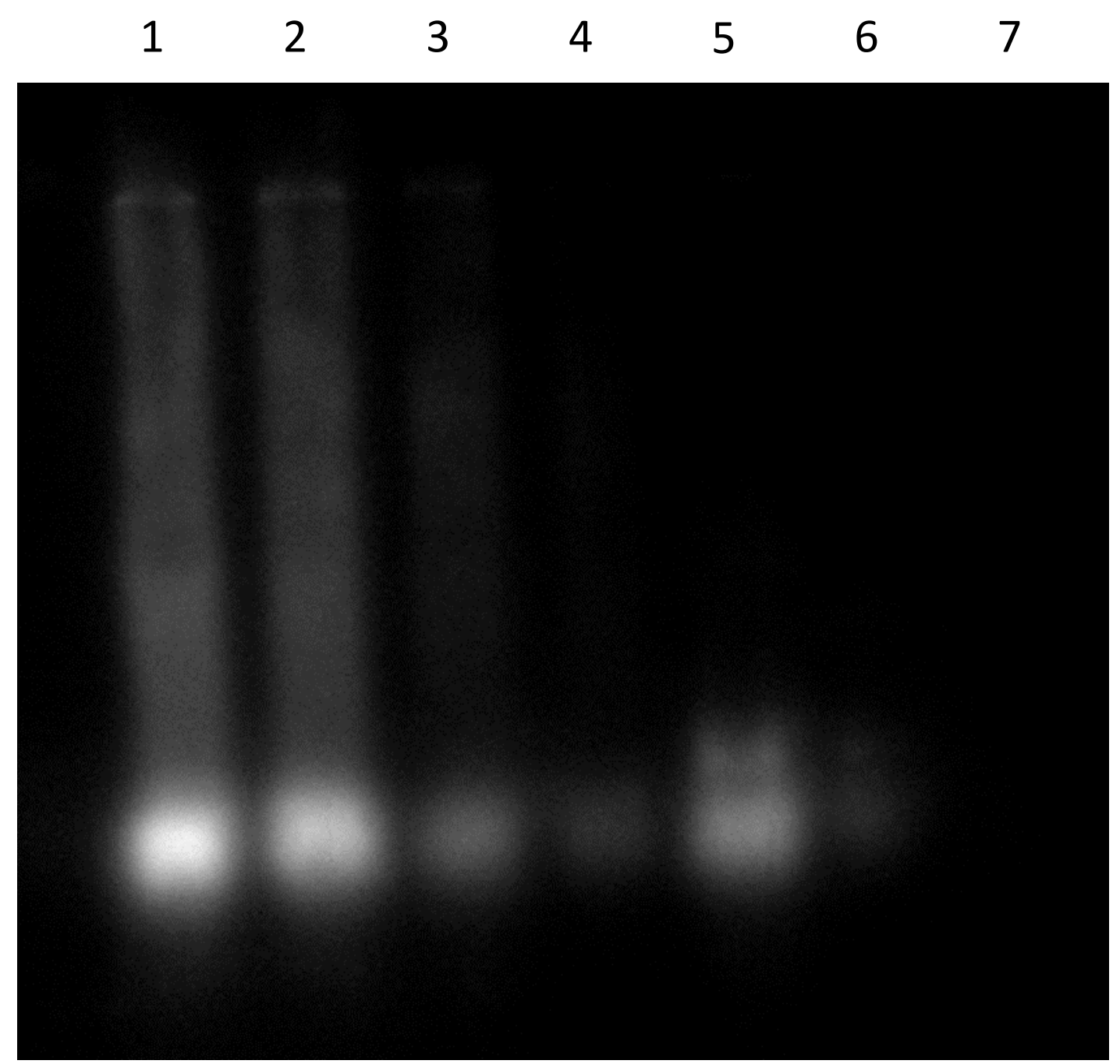

Figure 6. A 5\% bis-tris gel (50\% glycerol supplementation) monitoring the binding of

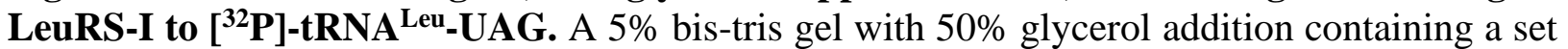
of serial diluted protein from lanes $1-7$. A total of $50 \mathrm{nM}$ of tRNA ${ }^{\mathrm{Leu}}-\mathrm{UAG}$ was utilized in addition to each of the protein samples. Lane 1 consists of $4.4 \mu \mathrm{M}$ final protein, lane 2 equates to $2.2 \mu \mathrm{M}$ final protein and subsequent lanes follow 2 -fold dilutions. Lane 7 consists of a final protein concentration of $0.0335 \mu \mathrm{M}$. 


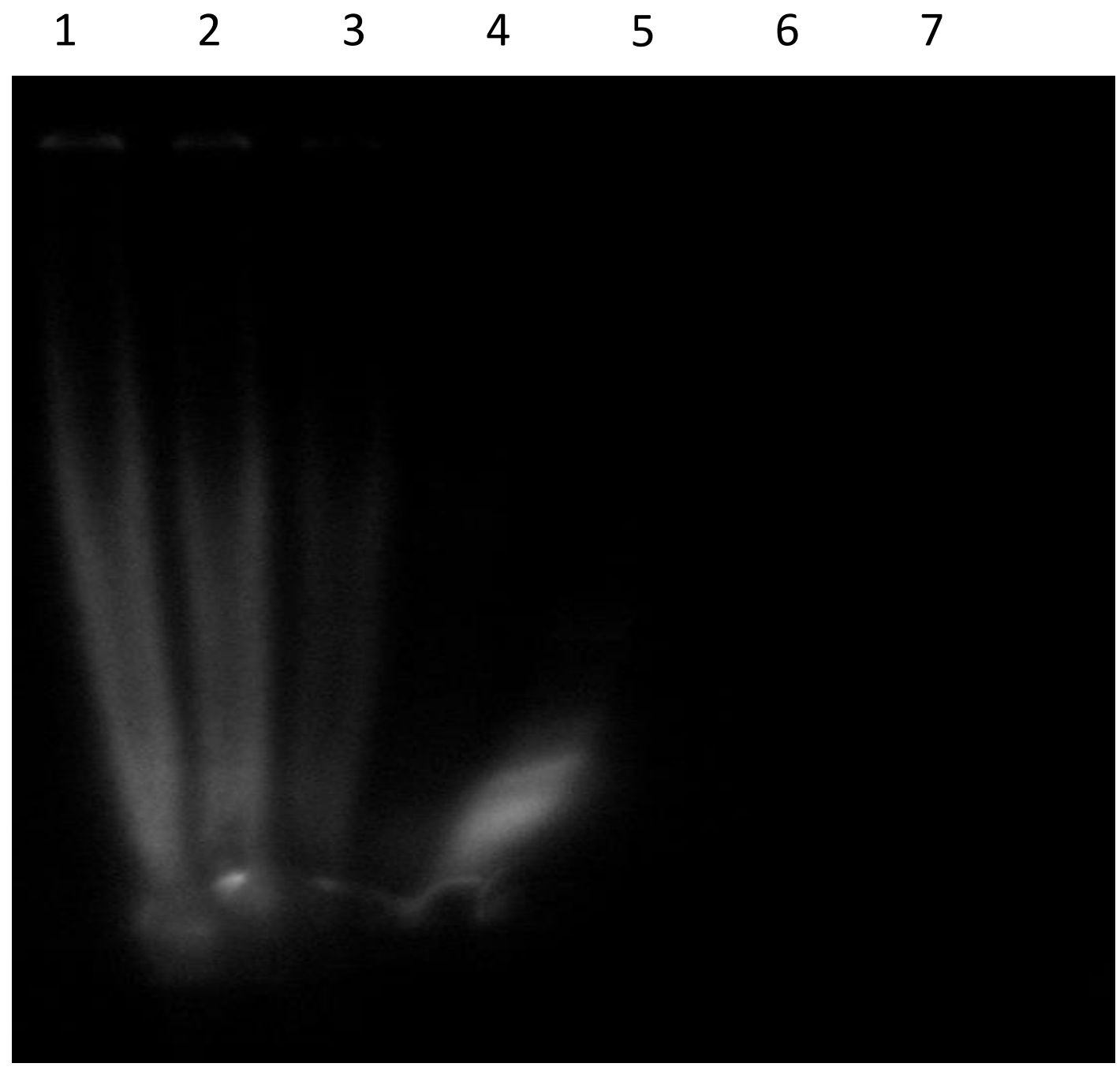

Figure 7. A $5 \%$ bis-tris gel (20\% TEG) monitoring the binding of LeuRS-I to [ $\left.{ }^{32} \mathrm{P}\right]-\mathrm{tRNA}^{\mathrm{Leu}}$ UAG. A $5 \%$ bis-tris gel supplemented with $20 \%$ TEG containing a set of serial diluted protein

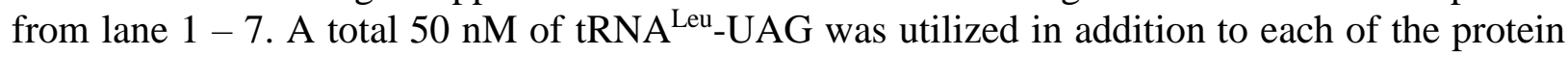
samples. Lane 1 consists of $4.4 \mu \mathrm{M}$ final protein, lane 2 equates to $2.2 \mu \mathrm{M}$ final protein and subsequent lanes follow 2-fold dilutions. Lane 7 consists of a final protein concentration of 0.0335 $\mu \mathrm{M}$. 


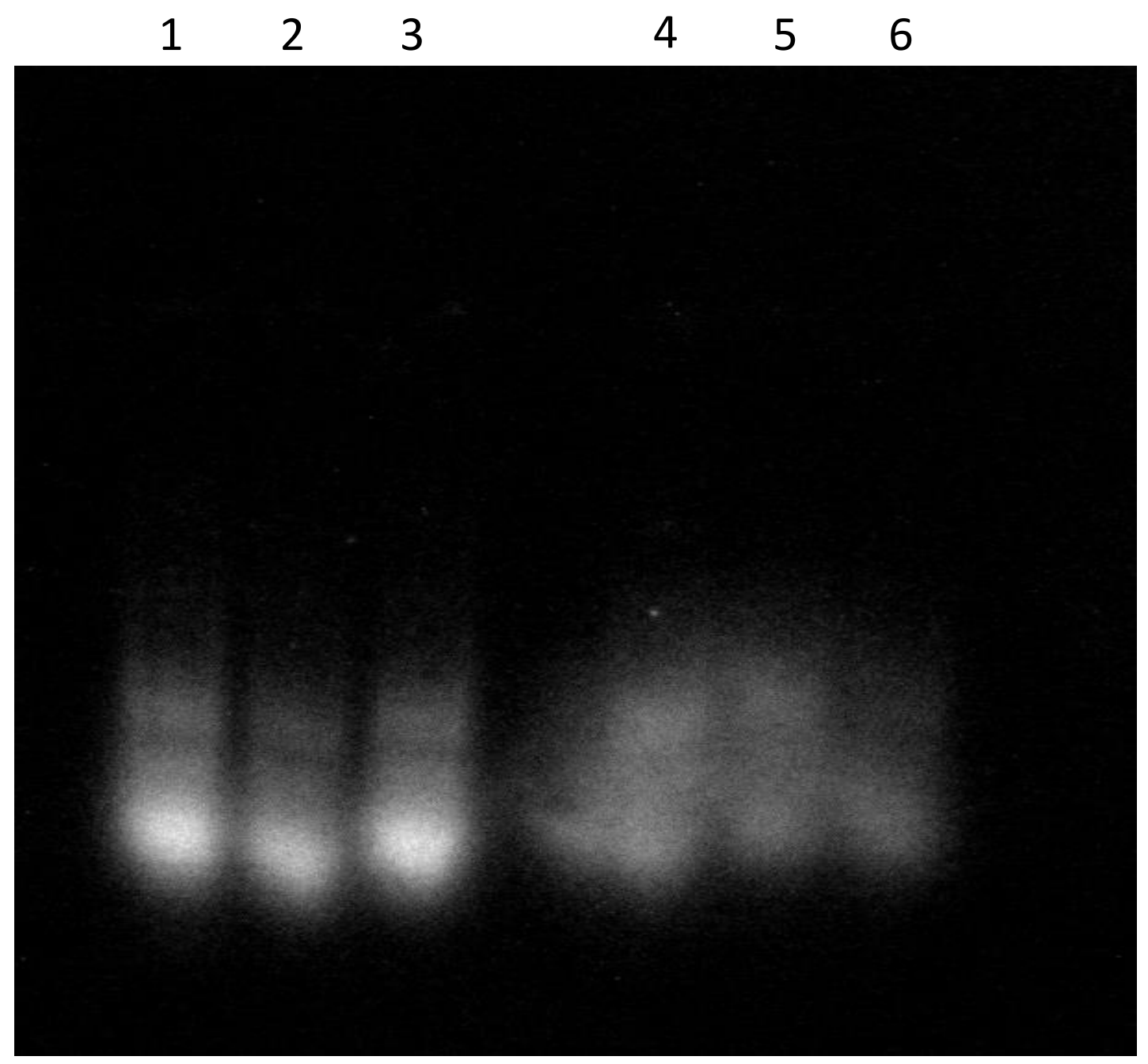

Figure 8. A 5\% bis-tris gel (37.5:1 acrylamide:bisacrylamide) monitoring the binding of

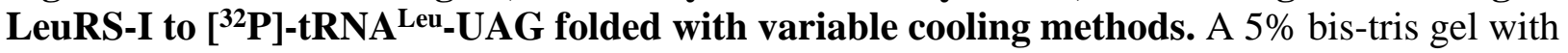
variable folding parameters. Lanes 1-3 pertain to the standard folding procedure, while lanes 4 6 correspond to snap-chilled folding mixes. A total $50 \mathrm{nM}$ of tRNA ${ }^{\text {Leu }}-\mathrm{UAG}$ was utilized in addition to each of the protein samples. Lane 1 consists of $4.4 \mu \mathrm{M}$ final protein, lane 2 equates to $2.2 \mu \mathrm{M}$, and lane 3 equates to $1.1 \mu \mathrm{M}$ final protein. These concentrations are mirrored for lanes 4 $-6$ 


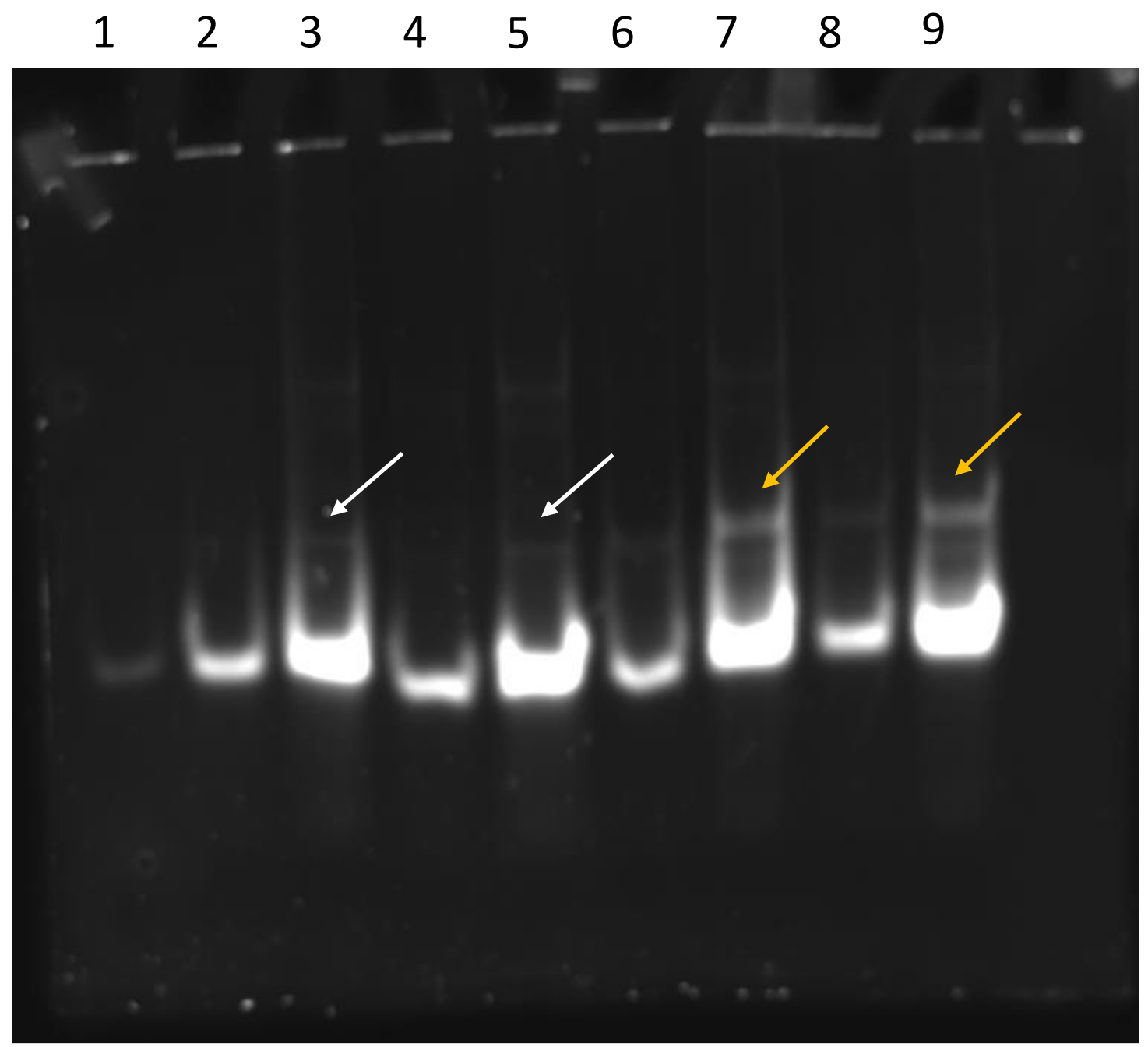

Figure 9. 5\% TBM (37.5:1 acrylamide:bisacrylamide) gel stained with SYBR Gold monitoring folded and unfolded cold tRNA ${ }^{\mathrm{Leu}}-\mathrm{UAG}$ in the presence and absence of supplemental buffer. A 5\% TBM gel containing different concentrations of cold UAG and varied incorporation of supplemental buffer. Lane assignments are as follows: $1.50 \mathrm{nM}(17.6 \mathrm{ng})$ folded UAG "+" supplemental buffer, 2. $400 \mathrm{nM}(141 \mathrm{ng})$ folded UAG "+" supplemental buffer, 3. $2400 \mathrm{nM}(843 \mathrm{ng})$ folded UAG "+" supplemental buffer, 4. $400 \mathrm{nM}(141 \mathrm{ng})$ folded UAG "-“" supplemental buffer, 5.2400 nM (843 ng) folded UAG “_“" supplemental buffer, 6. $400 \mathrm{nM}$ (141 ng) unfolded UAG "+” supplemental buffer, 7. $2400 \mathrm{nM}$ (843 ng) unfolded UAG “+” supplemental buffer, 8.400 nM (141 ng) unfolded UAG “-_" supplemental buffer, and 9. $2400 \mathrm{nM}$ (843 ng) unfolded UAG "-“" supplemental buffer. Indication of decreased alternative RNA structure between folded (white arrows) and unfolded (yellow arrows) tRNA ${ }^{\text {Leu-UAG of the same }}$ concentration noted. 


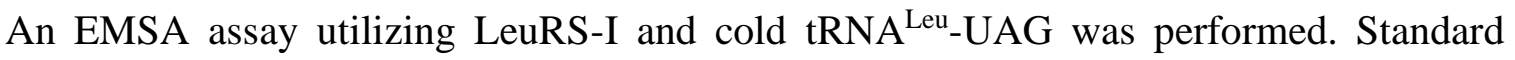
supplemental buffer and folding mix concentrations as noted previously were applied and the reactions were separated on a 5\% TBM gel and stained using SYBR Gold (Figure 10). Following staining and imaging of the gel, a clear shift was observed even though a corresponding decrease in the signal of the free tRNA band was not easily recognizable (Figure 10, Lanes $1-6$ ). It was also apparent that the shifted band also approached the well as protein concentration increased (Figure 10, compare Lanes 1 and 5). Taken together, we suspected that the SYBR Gold stain also was capable of detecting protein and that the shifted band migrated closer to the wells with increasing protein as a binding event was occurring (Figure 14, notice slight decrease in free tRNA from Lanes $4 \rightarrow 1$ ).

A secondary gel containing only protein was run to discern whether the SYBR Gold was binding to protein and giving a false impression of a gel shift. Standard reactions were assembled except excluding the cold tRNA ${ }^{\mathrm{Leu}}-\mathrm{UAG}$ substrate. These reactions were separated on a $5 \%$ (37.5:1 acrylamide:bisacrylamide) TBM gel and stained with SYBR Gold. As can be seen, SYBR Gold does, indeed, bind to LeuRS-I (Figure 11, arrow) as indicated by a fluorescent band near the gel wells, primarily in Lanes $1-6$. Interestingly, although the same concentrations of protein were used in these tRNA-less EMSA reactions, the staining was largely decreased (compare Figures 10 and 11). In addition, this experiment revealed a suspected problem using EMSA to gauge the interactions between our LeuRS paralogs with tRNA ${ }^{\mathrm{Leu}}$. From Figure 11 it is evident that LeuRSI fails to migrate appreciably into our gels using our gel and buffering system, explaining why binding has likely been observed with these proteins and tRNA with no discernable gel shift. 
To assess the problem regarding the lack of mobility of both LeuRS-F and LeuRS-I in both of the tested gel and buffer environments, a 22x20 short plate $8 \%$ TBM sequencing gel consisting of 75:1 acrylamide:bisacrylamide with $0.4 \mathrm{~mm}$ spacers was run containing LeuRS-I, LeuRS-F and E. coli LeuRS (Figure 12). Lanes 1-6, which comprised a serial diluted set of LeuRS-F, showed the most resilience to migration, even under high voltages for long periods of time ( 8 hours). The same appeared evident in lanes 7 - 12 which consisted of serial diluted LeuRS-I. E. coli LeuRS appeared to possess the best ability to migrate within the gel and buffering system tested as evident from lanes 13 - 18. Interestingly, the rate of migration of each protein perfectly matches expectations based off their predicted isoelectric points: LeuRS-F, 7.1; LeuRS-I, 5.6; E. coli LeuRS, 5.2. 


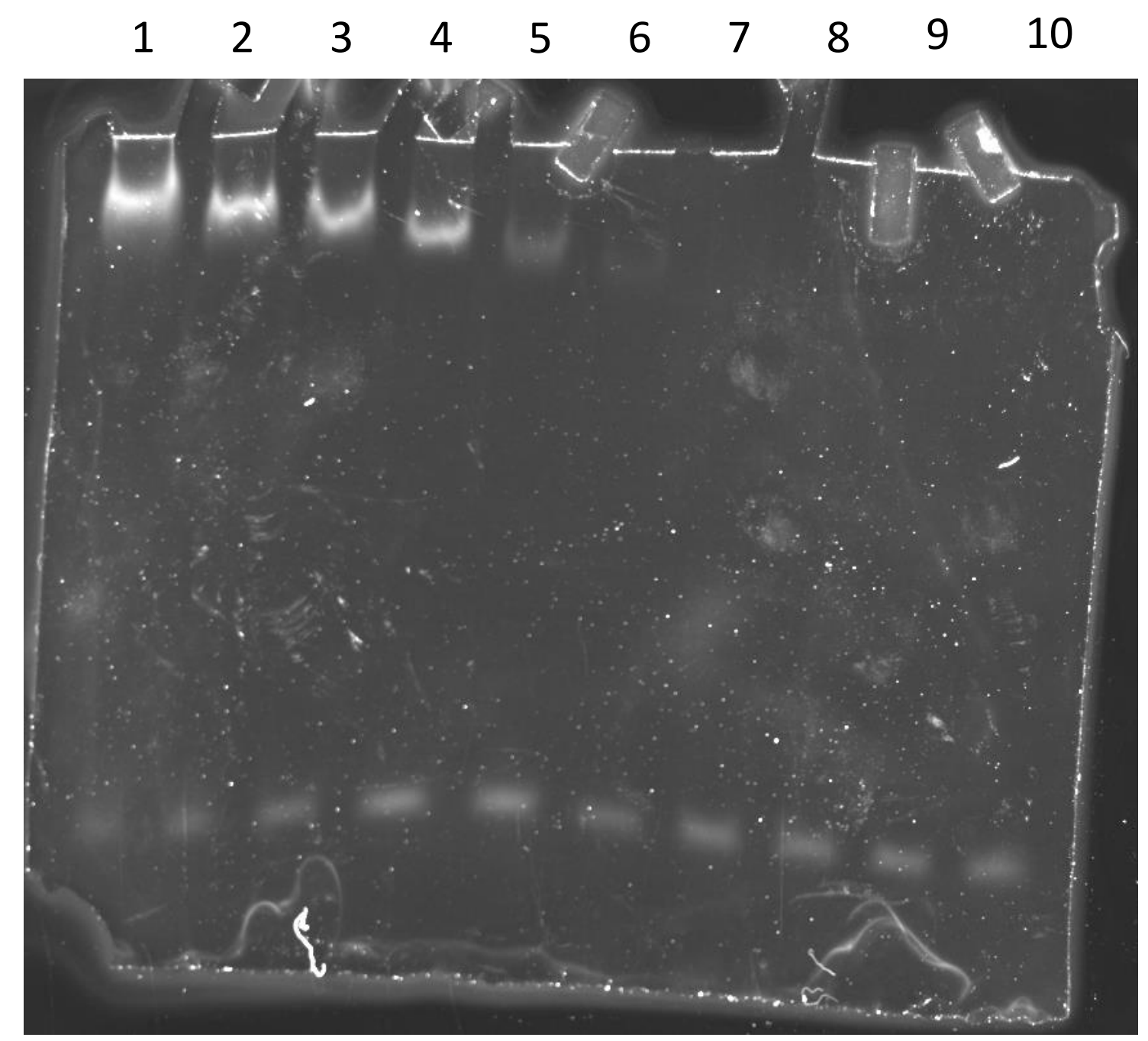

Figure 10.5\% TBM gel stained with SYBR Gold monitoring binding between LeuRS-I and

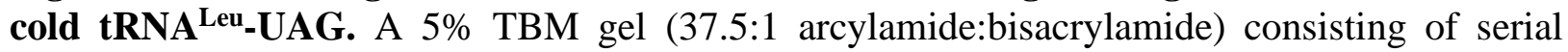
diluted protein and cold UAG folding mix. supplemental buffer consisting of $295 \mu \mathrm{M} \mathrm{MES,} \mathrm{pH}$ 6.7, $75 \mu \mathrm{M} \mathrm{ZnSO}_{4}$ and $20 \mu \mathrm{M} \mathrm{NaCl}$ and a $10 \mathrm{x}$ folding mix comprised of $10 \mu \mathrm{M}$ non-radiolabeled tRNA $_{\text {leu}}-\mathrm{UAG}$ (cold UAG), $5 \mu \mathrm{M} \mathrm{MgCl}_{2}, 10 \mu \mathrm{M}$ MES. A total of $50 \mathrm{nM}$ of tRNA ${ }^{\text {Leu-UAG was }}$ utilized in addition to each of the protein samples. Lane 1 consists of $4.4 \mu \mathrm{M}$ protein, lane 2 equates to $2.2 \mu \mathrm{M}$ and subsequent lanes follow 2-fold dilutions. Lane 9 consists of a final protein concentration of $0.0085 \mu \mathrm{M}$, with lane 10 serving as a tRNA control devoid of protein. 


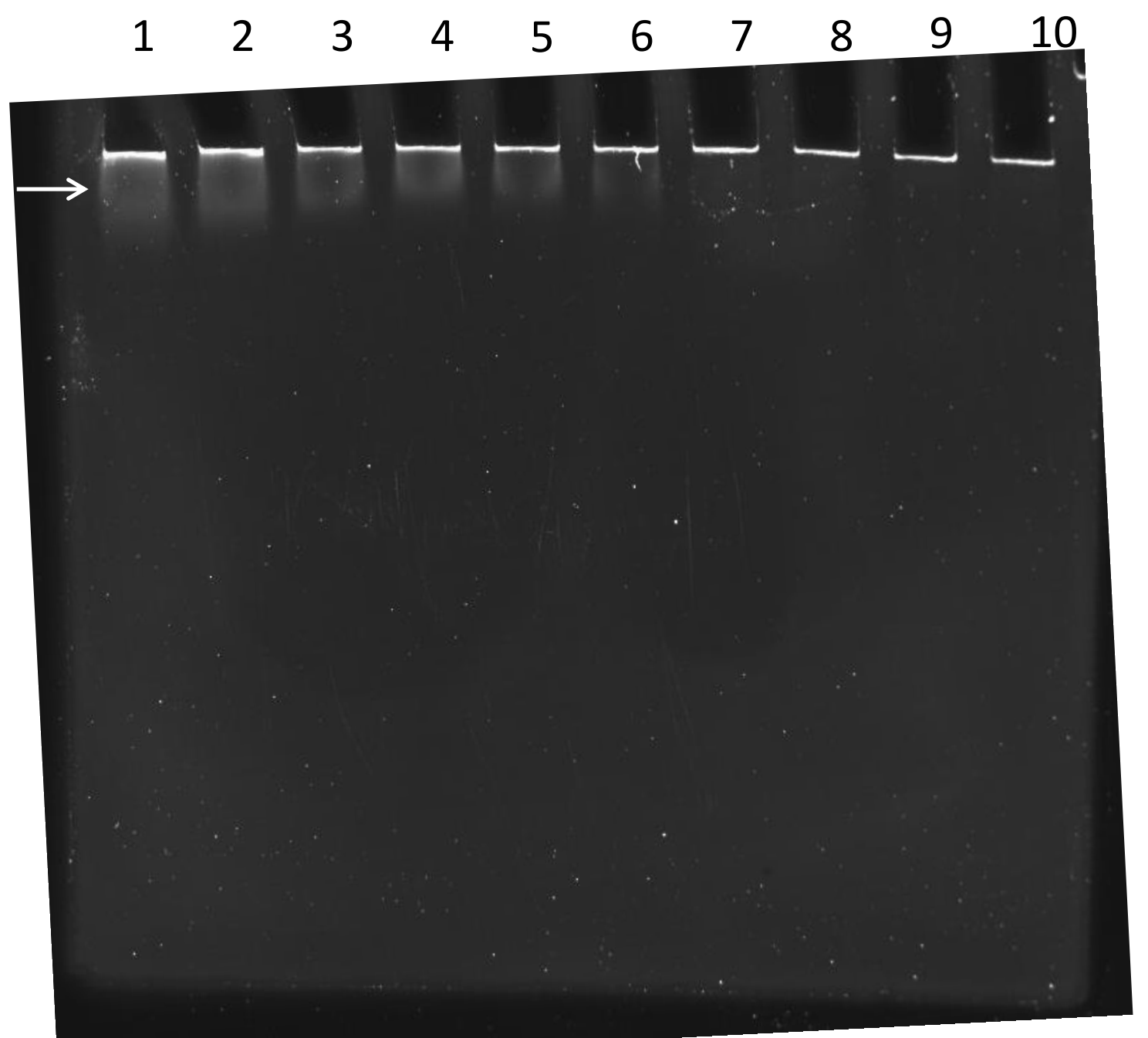

Figure 11. 5\% TBM gel of LeuRS-I, sans tRNA substrate stained with SYBR Gold. A 5\% TBM gel (37.5:1 acrylamide:bisacrylamide) consisting of serial diluted protein devoid of

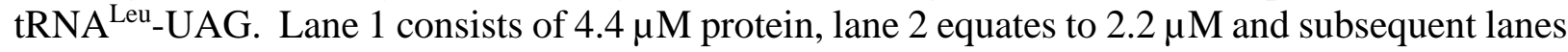
follow 2 -fold dilutions. Lane 10 consists of a final protein concentration of $0.00425 \mu \mathrm{M}$. Indication of stained protein at the eminence of the well is indicated (arrow). 


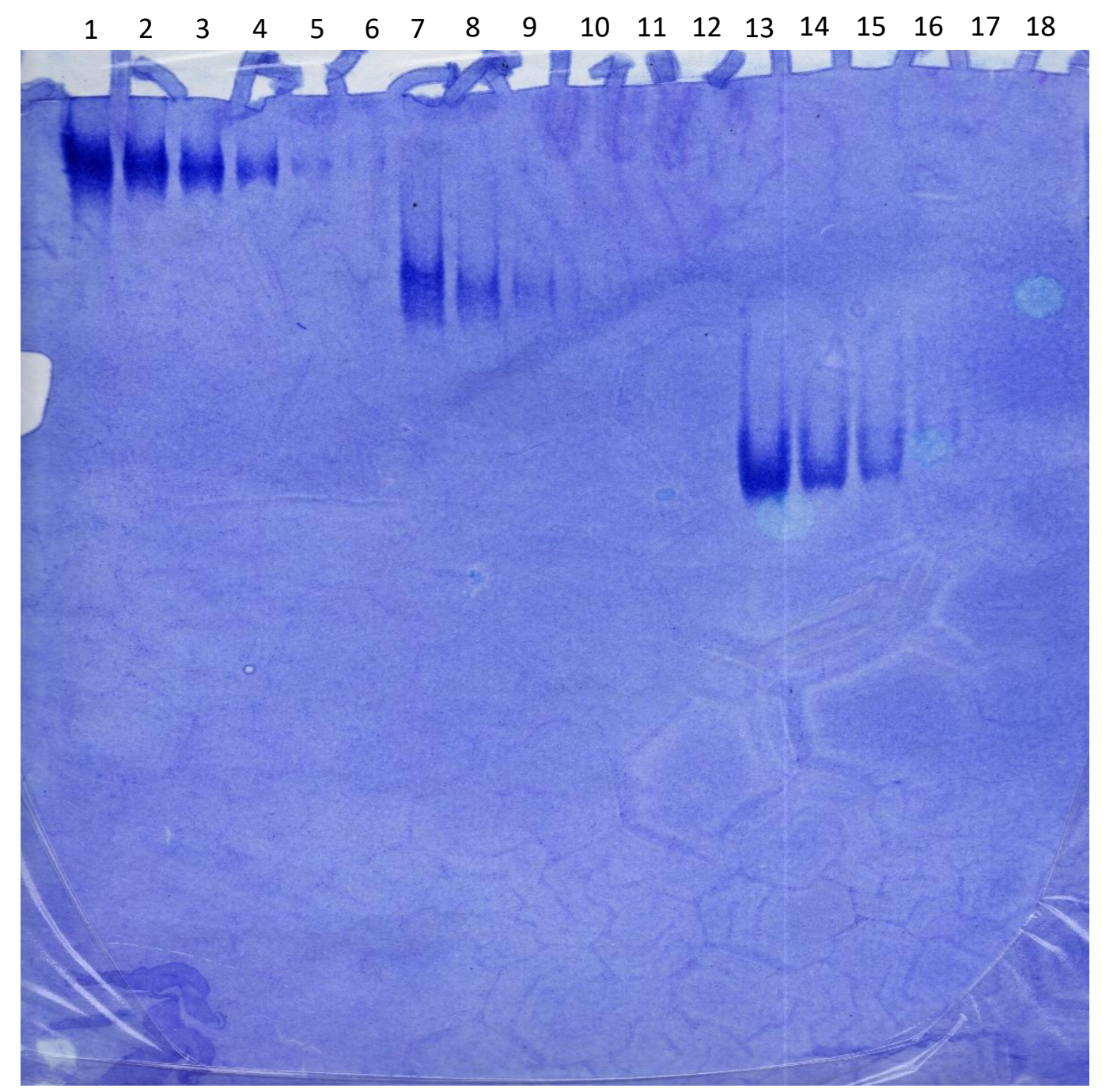

Figure 12. An 8\% TBM 75:1 acrylamide:bisacrylamide native PAGE sequencing gel containing serial diluted LeuRS-F, LeuRS-I, and $\boldsymbol{E}$. coli LeuRS. An 8\% TBM 75:1 sequencing gel (dimensions 22x20) of LeuRS-F, LeuRS-I and E. coli LeuRS (left to right). The gel was run at $800 \mathrm{~V}$ for 8 hours in a cold room to reduce heat. Lanes $1-6$ consist of high concentration LeuRS-F at an undiluted concentration of $82.7 \mu \mathrm{M}$ total load, with subsequent lanes being diluted 2-fold. Lanes $7-12$ portray LeuRS-I at an undiluted concentration of $22 \mu \mathrm{M}$ total load, with following lanes diluted 2-fold sequentially. Lanes $13-18$ consist of $E$. coli LeuRS at an initial undiluted concentration of $52 \mu \mathrm{M}$ total load, with subsequent lanes diluted 2-fold. 


\section{Microscale thermophoresis}

Because EMSAs were clearly not an optimal technique for gauging interactions between S. islandicus LeuRS paralogs and their tRNA substrates, we shifted our attention to microscale thermophoresis. This process typically monitors the movement of molecules within temperature gradients and can determine the binding interaction between a set of molecules. ${ }^{[51]}$ Due to the intrinsic fluorescent characteristics of both LeuRS-F and LeuRS-I decreasing when titrated with higher tRNA substrate concentrations, standard microscale thermophoresis was deemed as impossible and, therefore, a secondary method for detecting binding was required. As a workaround to this issue, it was deemed that fluorescence quenching of tryptophan residues on the proteins could be used to gauge the binding interaction of the protein to tRNA substrate. Binding reactions were made using $15 \mathrm{mM} \mathrm{MgCl}_{2}, 10 \mu \mathrm{M} \mathrm{ZnSO}_{4}, 25 \mathrm{mM} \mathrm{NaCl}, 2 \mathrm{mM}$ DTT, $12.5 \%$ glycerol, 60mM MES (pH 6.5), 0.1\% pluronic F-127, in vitro transcribed tRNA ${ }^{\text {Leu }}$-UAG, and 500nM LeuRS-F or LeuRS-I, along with a gradient of tRNA concentrations from $75 \mu \mathrm{M}$ to 0.59 $\mu \mathrm{M}$. The tryptophan residues present on LeuRS-F and LeuRS-I are provided: [LeuRS-F = 19; LeuRS-I $=11]$. Fluorescence quenching results indicated that, shockingly, both LeuRS-F and

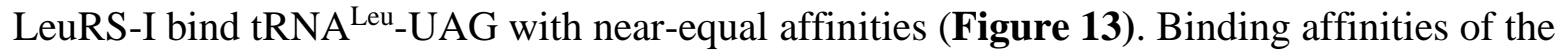

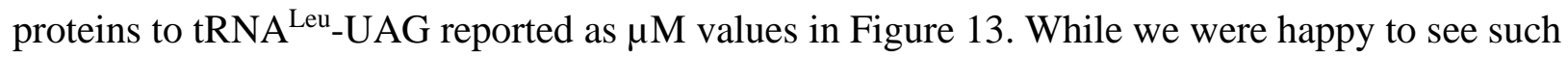

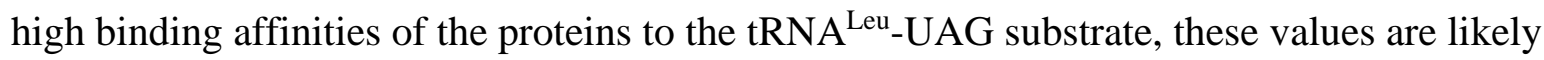
inflated by a magnitude which could either be resultant of the technique of fluorescence quenching of tryptophan residues or the artificially high tRNA concentrations used within the experiment. EMSA assays suggest that binding affinities should be around $5-10 \mu \mathrm{M}$. 


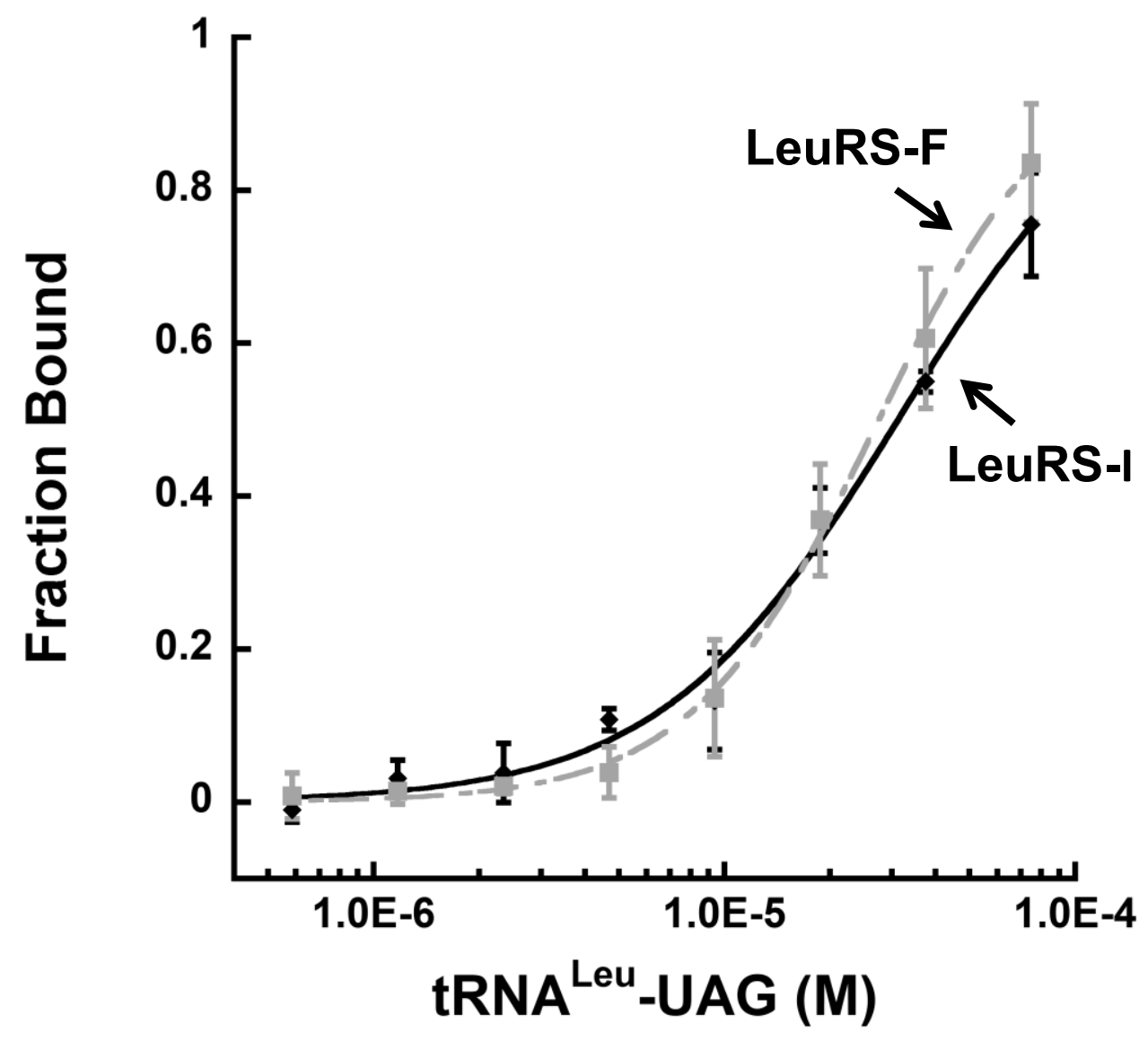

tRNA ${ }^{\text {Leu }}$-UAG $K_{D}(\mu \mathrm{M})$

LeuRS-F $\quad 27.1 \pm 5.8$

LeuRS-I $\quad 31.4 \pm 2.5$

Figure 13. Fluorescence quenching of tryptophan residues through protein-tRNA interaction. Fluorescence quenching was utilized to determine the binding activity between both LeuRS-F and LeuRS-I with tRNA ${ }^{\text {Leu }}$-UAG substrate. Reactions were proceeded with at room temperature, $\mathrm{pH} 6.5$ and contained $500 \mathrm{nM}$ of each protein. Folded tRNA ${ }^{\text {Leu }}-\mathrm{UAG}$ was added across three orders of magnitude from $75 \mu \mathrm{M}$ to $0.59 \mu \mathrm{M}$. Scans of fluorescence were fitted in the Hill equation to assess binding affinities. LeuRS-I (bold lines) and LeuRS-F (grayed lines) maintain similar binding affinities with tRNA substrate, with calculated binding affinities of LeuRS-F and LeuRS-I presented in $\mu \mathrm{M}$ values 


\section{tRNA Extraction}

The extraction of endogenous tRNA from $S$. islandicus is of great importance in potentially determining the activity of LeuRS-I within aminoacylation reactions. The possibility exists that LeuRS-I does not only bind tRNA ${ }^{\text {Leu }}$ but also has the capacity to bind non-cognate tRNA's and charge them with leucine. While initial aminoacylation reactions with LeuRS-I utilizing in vitro transcribed tRNA ${ }^{\text {Leu }}$ have yet to show positive activity, a pool of total tRNA with posttranscriptional modifications could alleviate this problem by offering a wide range of unique substrate for potential charging. Initial separation of tRNA from $S$. islandicus wild type strain was attempted using a generic lithium chloride extraction protocol provided by M.E. Saks, Senior Research Scientist of biochemistry at University of Illinois Urbana-Champaign. Extractions utilizing the basic $\mathrm{LiCl}$ protocol yielded moderate amounts of nucleic acid substrate as noted by nanodrop analysis (sample $S i$ wild type total tRNA-1; 10,617 ng/ $\mu \mathrm{L}$, Table A-1), but ultimately did not yield sufficient quantities of total tRNA for aminoacylation reactions as assessed by polyacrylamide sequencing gel analysis (Figure 14). Control samples of in vitro transcribed tRNA $^{\text {Leu }}-U A G$ were used as an indicator of relative size equal to that of the potential pool of total tRNA extracted from wild type cells, of which appeared minimal in lanes 3 and 4 of the sequencing gel. Even the vastly more abundant, Type I tRNAs were isolated in rather small yields (arrow,

Figure 14). However, contamination of high molecular weight nucleic acid was clearly present in these extraction samples and can be visualized near the wells of the sequencing gel within the two extraction samples, potentially indicating a source for the moderately high concentration of 10,617 $\mathrm{ng} / \mu \mathrm{L}$ found via nanodrop analysis.

A secondary $12 \%$ sequencing gel was assembled and run with samples of tRNA ${ }^{\text {Leu }}$-UAG, loading both $1 \mu \mathrm{g}$ and $2 \mu \mathrm{g}$ quantities, as well as freshly extracted E. coli tRNA ( $2 \mu \mathrm{g})$ and $S i$ total 
tRNA-1 $(2 \mu \mathrm{g})$ (Figure 15). The E. coli total tRNA extraction yielded the expected total pool of tRNA at around $\sim 88 \mathrm{nt}$ of size which correlated to roughly the same size as the in vitro transcribed

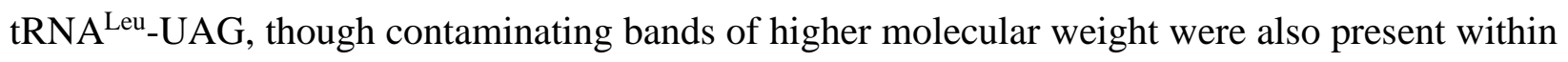
this sample. Si total tRNA-1 still showed a lack of tRNA, and a secondary extraction of wild type S. islandicus cells was required.

A secondary extraction of S. islandicus wild type cells was performed using an increased volume of cells grown at $75^{\circ} \mathrm{C}$. Si total tRNA-2 was run in tandem with $E$. coli total tRNA as a comparative sample on a $2 \%$ agarose bleach gel. From this gel as well as the NanoDrop analysis (sample $S i$ total tRNA-2; 20,081 ng/ $\mathrm{LL}$, Table A-1), it is clear that when using smaller volumes of the initial $\mathrm{LiCl}$ and phenol:chloroform:isoamyl alcohol reagents, extractions yielded greater quantities of total tRNA at a superior quality (Figure 16).

Higher molecular weight nucleic acid species were also present in the Si total tRNA-2 sample, which was a trend seen within every extraction performed. These contaminating nucleic acid species required removal for downstream applications such as aminoacylation where knowing the actual tRNA concentration is crucial. E. coli total tRNA was utilized as a substrate to test a new chemical extraction method for removing these unwanted contaminants. Utilizing raw $\mathrm{LiCl}$ reagent and subsequent ethanol cleanup, E. coli total tRNA was fractionated from large contaminating nucleic acids (Figure 17). 


\section{$\begin{array}{llll}1 & 2 & 3 & 4\end{array}$}

Figure 14. 12\% sequencing gel analysis of $\mathrm{Si}$ wild type total tRNA and

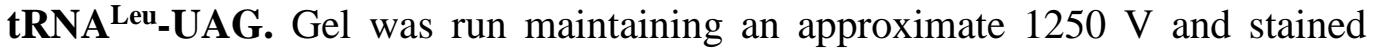
using Stains-All. Lane assignments are as follows: 1. $1 \mu \mathrm{g} S i$ tRNA-1, 2. $2 \mu \mathrm{g}$ of $S i$ tRNA-1, 3. $1 \mu \mathrm{g}$ tRNA ${ }^{\text {Leu }}$-UAG, and $4.2 \mu \mathrm{g}$ of tRNA ${ }^{\text {Leu }}-$ UAG. Isolated Type I tRNAs are indicated (arrow). 


\section{$\begin{array}{llll}4 & 3 & 2 & 1\end{array}$}

\section{S rRNA or mRNA $\longrightarrow$}

Type II tRNA's $\longrightarrow$

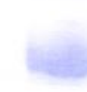

\section{.}




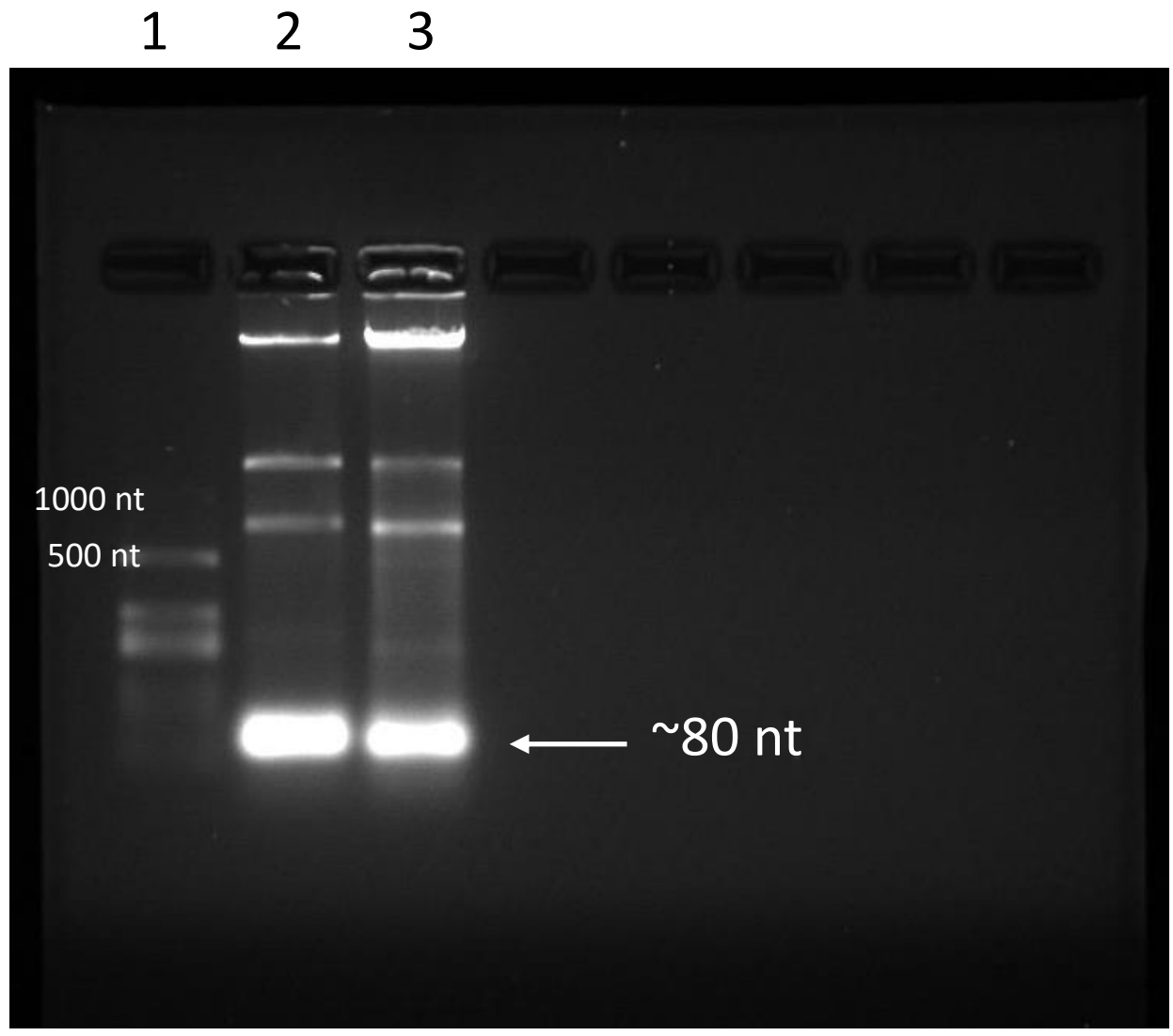

Figure 16. 2\% agarose bleach gel of $E$. coli total tRNA and $S i$ total tRNA. Agarose bleach gel run in 1x TAE buffer at a constant $90 \mathrm{~mA}$ for 30 minutes. Staining achieved by the addition of $0.5 \mu \mathrm{g} / \mathrm{mL}$ ethidium bromide. Lane assignments are as follows 1. Low range ssRNA ladder, 2. 2 $\mu \mathrm{g}$ E. coli total tRNA, 3. $2 \mu \mathrm{g}$ Si total tRNA-2. Arrow indicates pool of total tRNA at around roughly $80 \mathrm{nt}$ in size. Ladder is marked with weights of upper bands. 


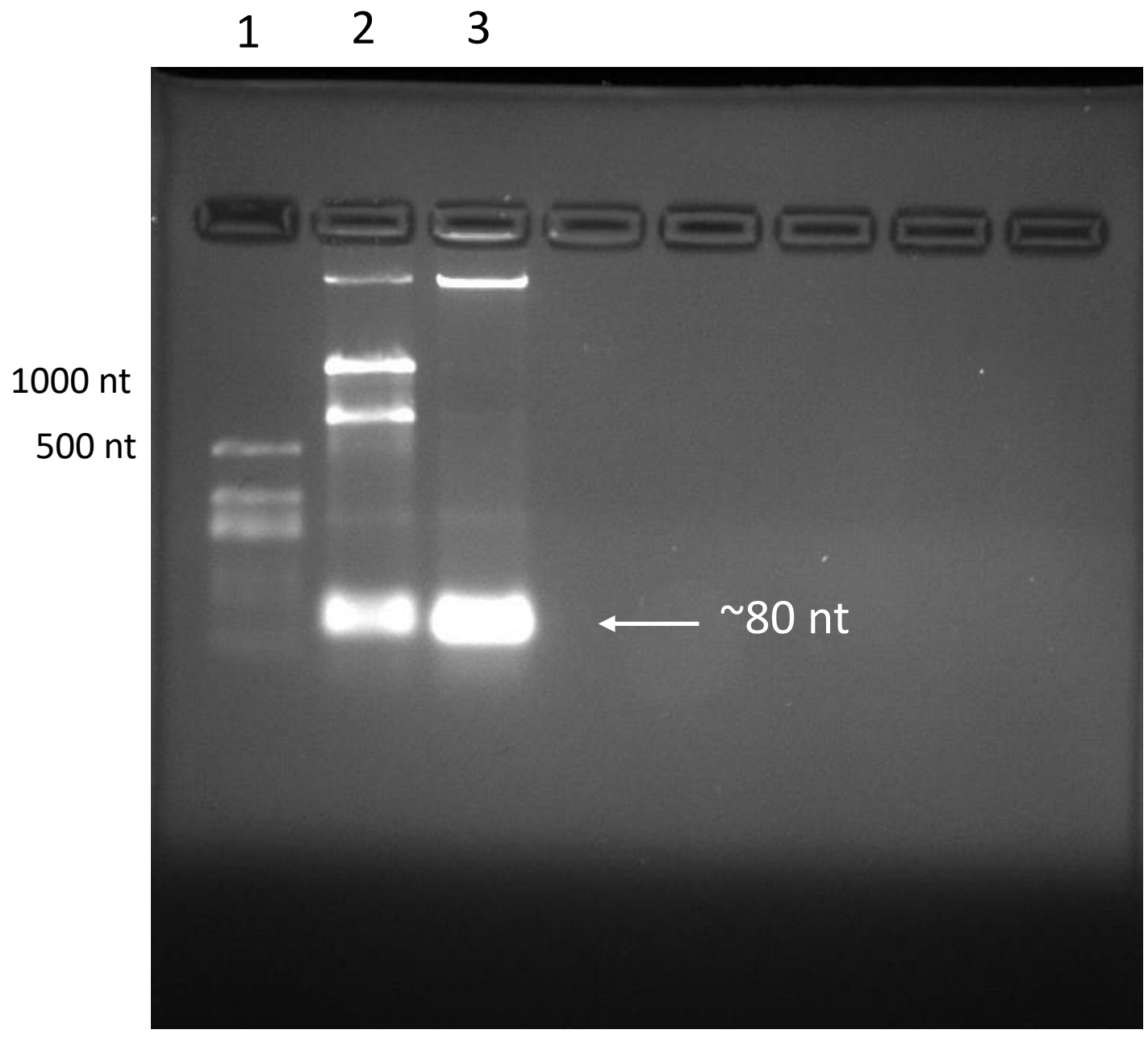

Figure 17. Fractionation of total $E$. coli tRNA from high molecular weight nucleic acids Agarose bleach gel run in 1x TAE buffer at a constant $90 \mathrm{~mA}$ for 30 minutes. Staining achieved by the addition of $0.5 \mu \mathrm{g} / \mathrm{mL}$ ethidium bromide. Lane assignments are as follows: 1 . Low range ssRNA ladder, 2. $2 \mu \mathrm{g}$ unprocessed $E$. coli total tRNA, 3. $2 \mu \mathrm{g}$ contaminant-extracted $E$. coli total tRNA. Size indication of the total pool of tRNA shown (arrow) as well as the top band sizes of the low range ssRNA ladder. 
Initial fractionation of $E$. coli total tRNA from high molecular weight nucleic acids was a reassuring move in the right direction, though a larger molecular weight band was still present in the extracted sample. As larger RNA species are insoluble in $\mathrm{LiCl}^{[47]}$, we assumed that the initial fractionation removed mRNA and 5S ribosomal RNA leaving behind genomic DNA within the tRNA sample. Therefore, a small amount of $S i$ total tRNA-2 was subsequently processed with DNase in an attempt to remove the high molecular weight band. As can be seen from Figure 18, an overnight digest at $37^{\circ} \mathrm{C}$ using 2 units of DNase effectively removed this contaminating species, confirming our suspensions. Importantly, these additional, straightforward procedural steps are highly effective at cleaning and enriching our RNA pool for total tRNA.

These additional steps were applied to the crude $S i$ total tRNA-2 pool. A bleach agarose gel (Figure 19) tracking the full process of contamination cleanup was run. As can be seen in lane 3 of the gel, a high-fidelity digestion of DNA was achieved by the addition of 2 units of DNase to the sample. A $\mathrm{LiCl}$ extraction of the remaining contaminating bands was attempted, but ultimately failed due to the improper addition of absolute ethanol during the extraction procedure (Figure 19, Lane 4) which ultimately precipitated both large and small RNAs. To remedy this, a secondary extraction using only $\mathrm{LiCl}$ reagent was performed, afterwhich much of the contaminating nucleic acids were removed from the pool of total tRNA (Figure 19, Lane 5).

A secondary extraction of crude $S i$ total tRNA was carried out at the stressful condition of $60^{\circ} \mathrm{C}$ to provide a secondary substrate for potentially eliciting activity within aminoacylation reactions involving LeuRS-I. This temperature was chosen for growth due to possible differences in the modification types present on the tRNA's at sub-optimal temperatures of growth, as noted by McCloskey and cohort within the hyperthermophilic Methanococcales. ${ }^{[48]}$ These potential differences in modification could be required for activity elicitation with LeuRS-I in 
aminoacylation reactions. Along with this, it is possible that LeuRS-I works with non-cognate tRNA as is seen in mammalian systems with MetRS, ${ }^{[22]}$ providing further importance to performing low-temperature extractions. Utilizing the aforementioned processes of extraction and contaminant removal, this secondary extraction yielded high concentrations of relatively pure substrate (Table A-1 \& A-2) as shown in initial extraction samples $S i$ total tRNA-3 (11,080 ng/ $\mu \mathrm{L})$ and $\mathrm{Si}$ total tRNA-4 $(14,050 \mathrm{ng} / \mu \mathrm{L})$, as well as the purified and pooled $\mathrm{Si}$ total tRNA $\left(60^{\circ} \mathrm{C}\right)$ at $14,280 \mathrm{ng} / \mu \mathrm{L}$. Figure 20 is a bleach agarose gel tracking the aforementioned samples.

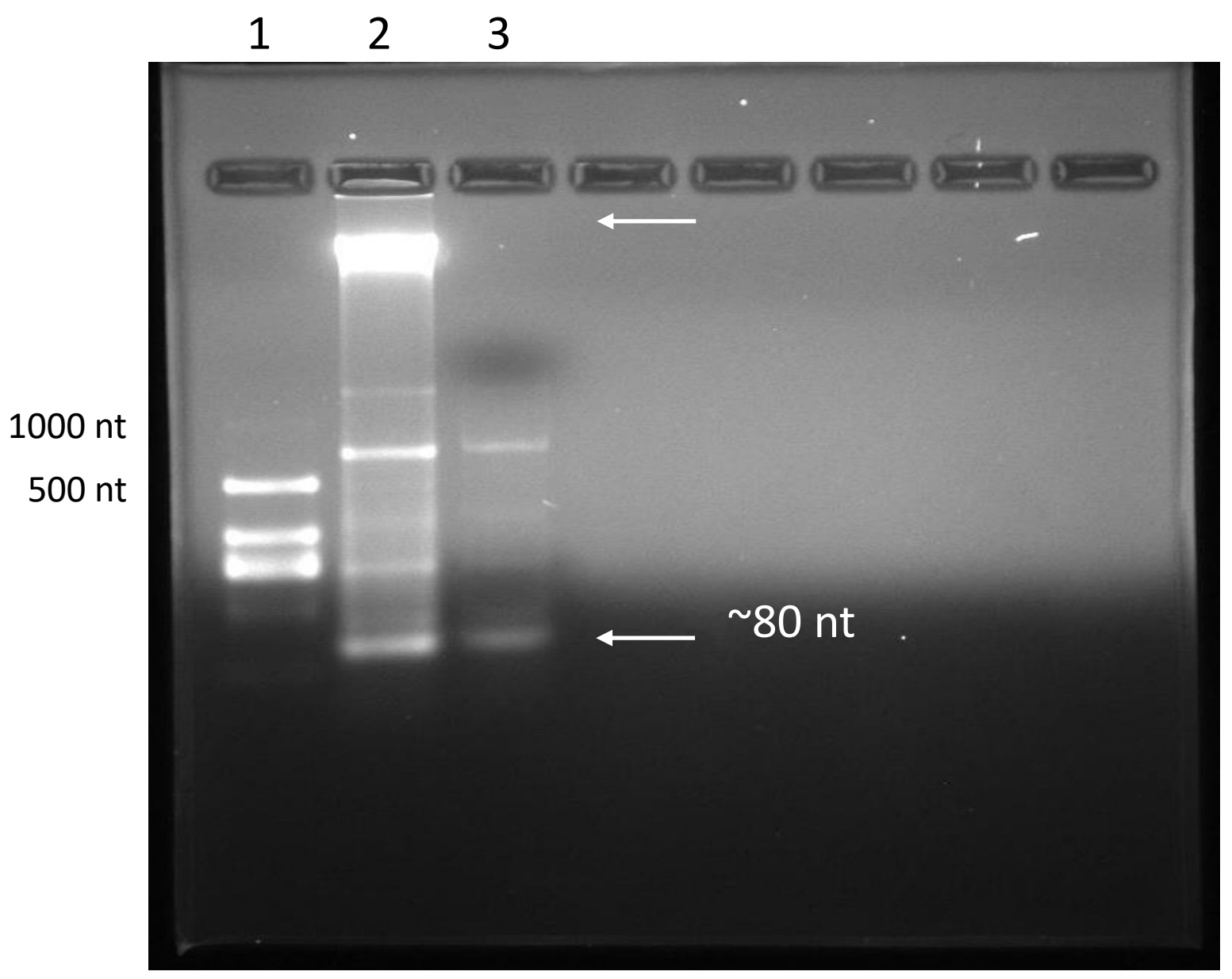

Figure 18. 2\% agarose bleach gel tracking the digestion of $S i$ total tRNA-2 with DNase. Agarose bleach gel run in $1 \mathrm{x}$ TAE buffer at a constant $90 \mathrm{~mA}$ for 30 minutes. Staining achieved by the addition of $0.5 \mu \mathrm{g} / \mathrm{mL}$ ethidium bromide. Lane assignments are as follows: 1. Low range ssRNA ladder, 2. Undigested Si total tRNA-2 at $\sim 1.5 \mu \mathrm{g}, 3$. DNase digested $S i$ total tRNA-2 at $\sim 0.8 \mu \mathrm{g}$. Successful digestion of DNA is apparent from the loss of signal indicated at the top of the gel (arrow). Ladder assignments in nucleotide are displayed for the top two bands. 


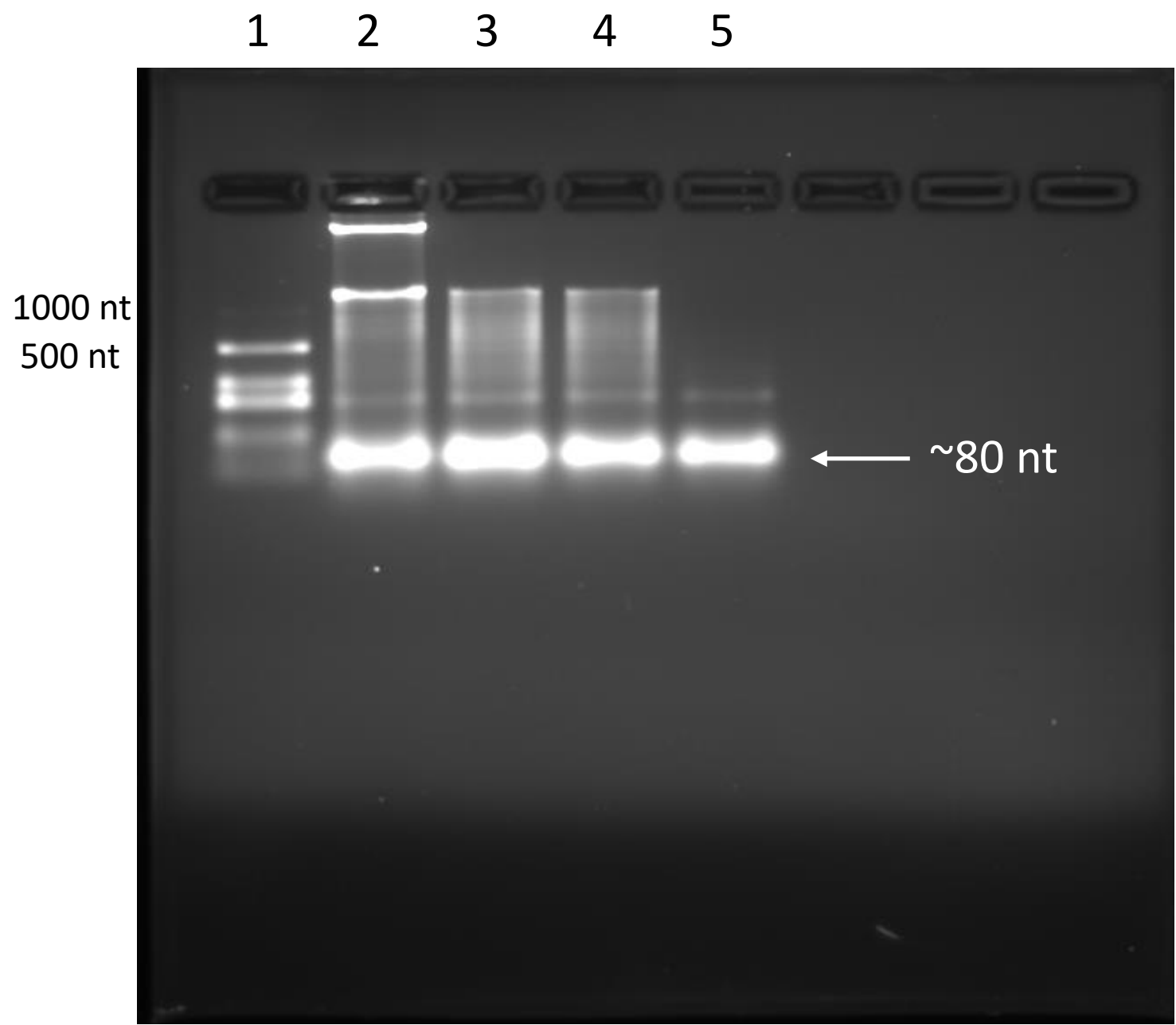

Figure 19. 2\% agarose bleach gel tracking contamination cleanup in Si total tRNA-2. Agarose bleach gel run in $1 \mathrm{x}$ TAE buffer at a constant $90 \mathrm{~mA}$ for 30 minutes. Staining achieved by the addition of $0.5 \mu \mathrm{g} / \mathrm{mL}$ ethidium bromide. Lane assignments are as follows: $\mathbf{1}$. Low range ssRNA ladder, 2. unprocessed extracted $S i$ total tRNA-2, 3. DNase digested Si total tRNA-2, 4. Failed $\mathrm{LiCl}$ extraction $\mathrm{Si}$ total tRNA-2, 5. Final processed $\mathrm{Si}$ total tRNA-2. All samples were standardized to $1 \mu \mathrm{g}$ of total loaded nucleic acid. 


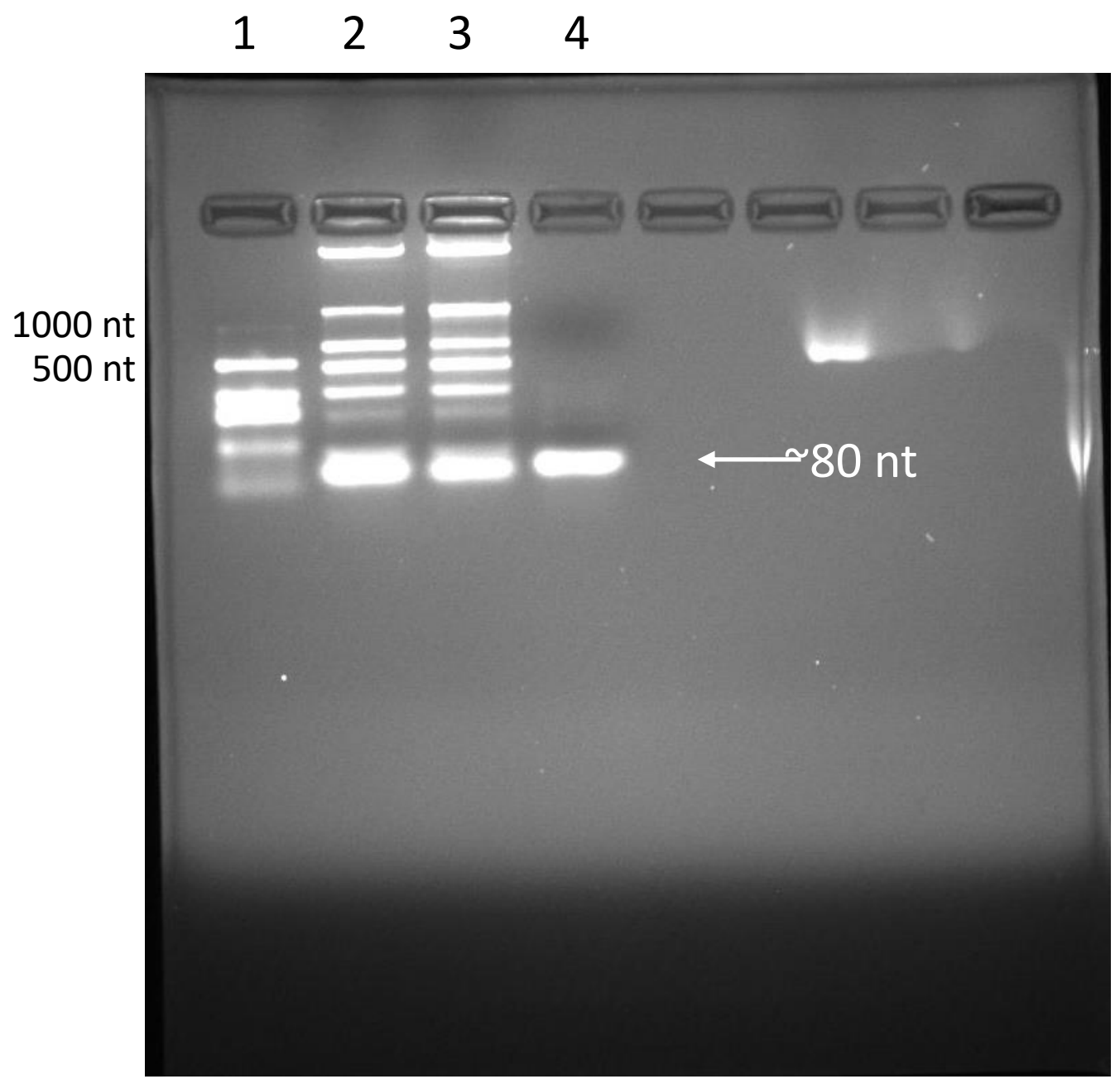

Figure 20. 2\% agarose bleach gel depicting $\mathrm{Si}$ total tRNA-4 \& 5 and final processed and pooled low temperature total tRNA. Agarose bleach gel run in 1x TAE buffer at a constant 90 $\mathrm{mA}$ for 30 minutes. Staining achieved by the addition of $0.5 \mu \mathrm{g} / \mathrm{mL}$ ethidium bromide. Lane assignments are as follows: 1. Low range ssRNA ladder, 2. $S i$ total tRNA-4, 3. Si total tRNA-5, 4. Purified low temperature total tRNA. Total pool of tRNA indicated at roughly 80 nucleotides (arrow) along with the sizes top bands of low range ssRNA. 


\section{LeuRS-I C-terminal domain expression and purification}

In an effort to understand the activity of LeuRS-I, tools for determining the expression levels of the protein in vivo are required. In order to do this, two distinct avenues for obtaining this result were taken - the development of custom polyclonal antibodies raised against the $\mathrm{C}$-terminal domain of LeuRS-I that are specific in their recognition of LeuRS-I but not LeuRS-F, and the generation of a wild type $S$. islandicus strain harboring a fusion tagged LeuRS-I protein. Both of these methods will allow us to determine both when and at what levels LeuRS-I is expressed and if this expression is modulated via stressful environmental conditions. These generation of selective antibodies also has the added benefit of determining whether LeuRS-I has a unique binding partner with which it makes interactions, such as LeuRS-F or another endogenous protein. For the development of polyclonal antibodies, it was determined that the C-terminal domain of LeuRS-I was the best candidate to raise the antibodies against due to this portion of the protein being most dissimilar to LeuRS-F (Figure 21).

This fragment containing $20 \%$ sequence identity to LeuRS-F was cloned into pET14b (+) (Novagen) and used to transform BL21(DE3) (NEB). It is important to note that both enzymes share a small block of amino acids that are very similar at the C-termini of each protein. The expression and affinity purification of the C-terminal domain (CTD) of LeuRS-I [His6-LeuRS-I (789-934)] was monitored via SDS-PAGE. After lysis via sonication, soluble lysates (Figure 22,

Lane 3) were loaded onto columns containing $\mathrm{Ni}^{2+}$-NTA resin which captures the His-tag fused to the N-terminus of the LeuRS-I CTD. Following several rinse steps (Figure 22, Lanes 5 - 8), the bound protein was eluted with a large volume of high concentration buffered imidazole leading to a seemingly low protein yield (Figure 22, Lane 9). The eluted protein was dialyzed and concentrated into a buffer suitable for thrombin digest, the aim of which is to remove the His-tag 
from His 6 -LeuRS-I(789-934) (Figure 22, Lane 10, $19.2 \mathrm{kDa}$, circled). As gauged from the gel, a moderate amount of the desired protein was acquired, and approximately 60\% pure (Figure 22, compare Lanes 3 and 10). Following the purification of the C-terminal domain, the total protein concentration was determined to $7.52 \mathrm{mg}$.

To remove the His-tag and generate a LeuRS-I CTD better approximating the wild type sequence, a thrombin digest was performed. Initial trials tested several molar ratios of thrombin:His6-LeuRS-I(789-934) over the course of 24 hours (data not shown). A large-scale digest was carried out and monitored via SDS-PAGE over the course of sixteen hours (Figure 23). While the thrombin digest was complete after 2 hours (Figure 23, compare arrows in Lanes 2 and 3 ), the cleaved protein migrated as a doublet. We speculate that this aberrant migration pattern is the result of thrombin miscleavage on a portion of the LeuRS-I fragment or that a subset of our purified protein had become oxidized. Regardless, the predicted molecular weight of our cleaved protein $(17.3 \mathrm{kDa})$ matches that observed within our SDS-PAGE analysis within the resolution of the experiment. However, incredibly pure protein is needed to raise a custom antibody and Figure $\mathbf{2 3}$ suggested that a secondary column was needed to further purify our cleaved LeuRS-I variant.

Because the thrombin utilized in cleavage reactions was biotinylated it was readily captured with streptavidin-coated beads (data not shown). Following this step, His-tags liberated from the cleavage reaction were removed by passing digested and thrombin-removed LeuRS-I (789-934) over $\mathrm{Ni}^{2+}$-NTA resin (Figure 24). This added step not only removed the unwanted and freed His-tags, but also removed considerable amounts of additional, contaminating protein species (Figure 24, compare Lane 1/3 with Lane 4). However, remaining contaminants with molecular weights hovering around $70-75 \mathrm{kDa}$ were still present. 


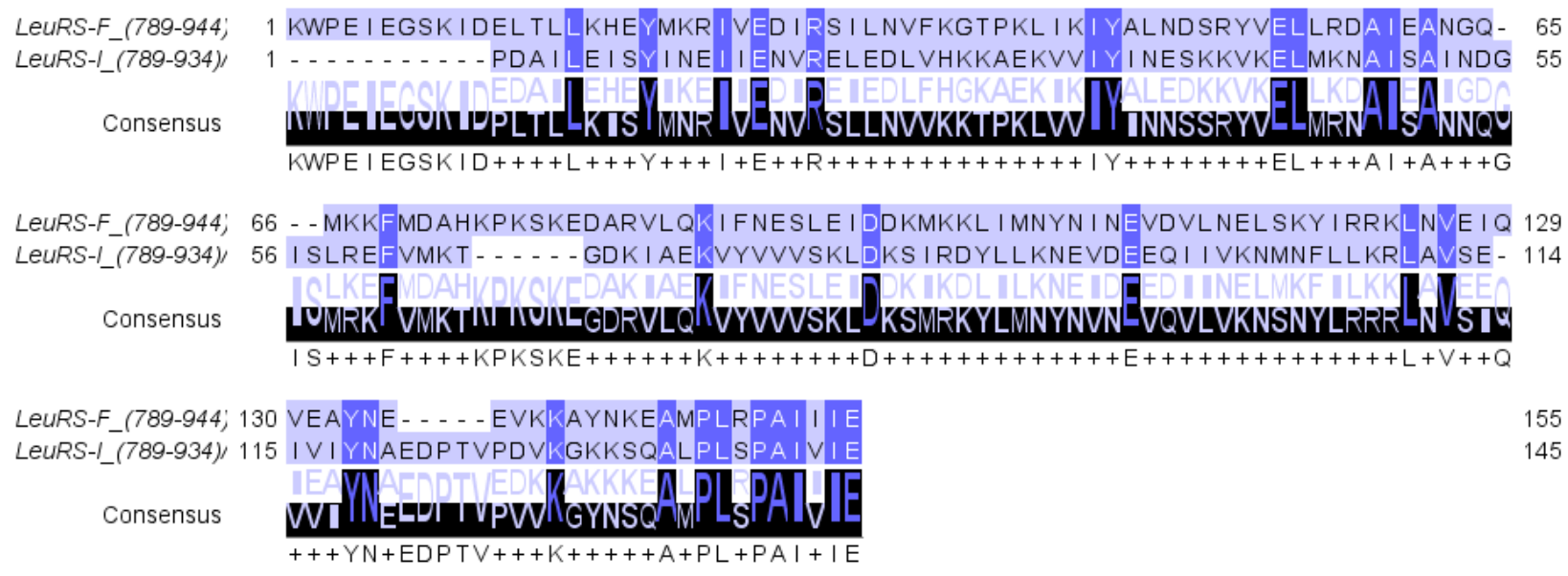

Figure 21. Sequence alignment between the C-terminal domain of LeuRS-I (residues 789934) and LeuRS-F (residues 789-944). Alignments of the C-terminal domains of each of these proteins indicative of lowest sequence identity (20\%) between the two proteins and was determined to be the best fit for raising polyclonal antibodies selective in their recognition of LeuRS-I. 


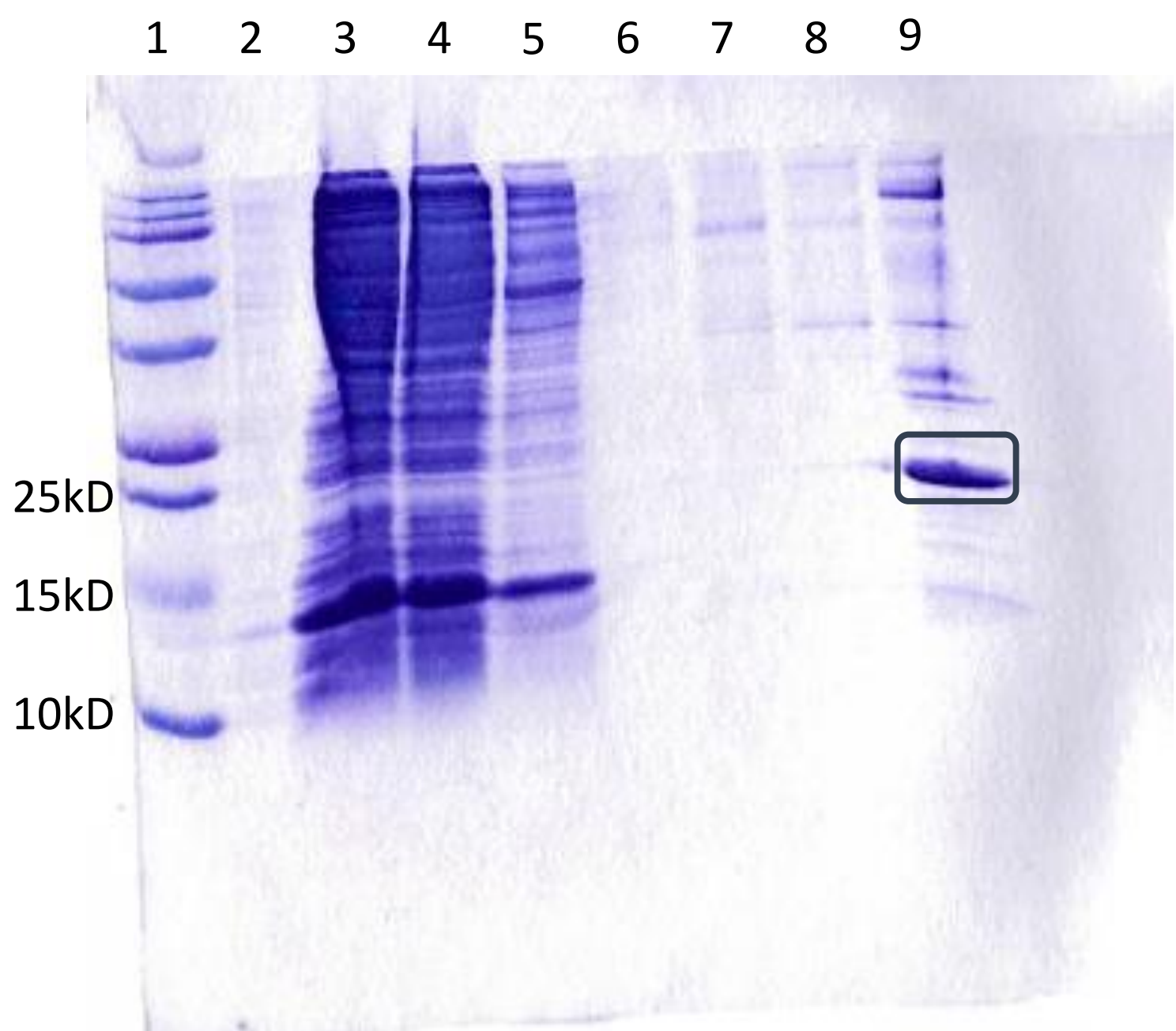

Figure 22. 15\% SDS-PAGE tracking the affinity purification of His6-LeuRS-I (789-934). Lane assignments are as follows: 1. $250 \mathrm{kDa}$ ladder (Goldbio), 2. sonicated cell mass, 3. supernatant of cleared lysate, $\mathbf{4}$. flowthrough, 5. lysis rinse, $\mathbf{6}$. imidazole rinse 1, 7. imidazole rinse 2, 8. final eluted C-terminal fragment, 9. dialyzed C-terminal fragment. Boxed protein indicated within the dialyzed fraction is the $19.2 \mathrm{kDa}$ C-terminal fragment. 


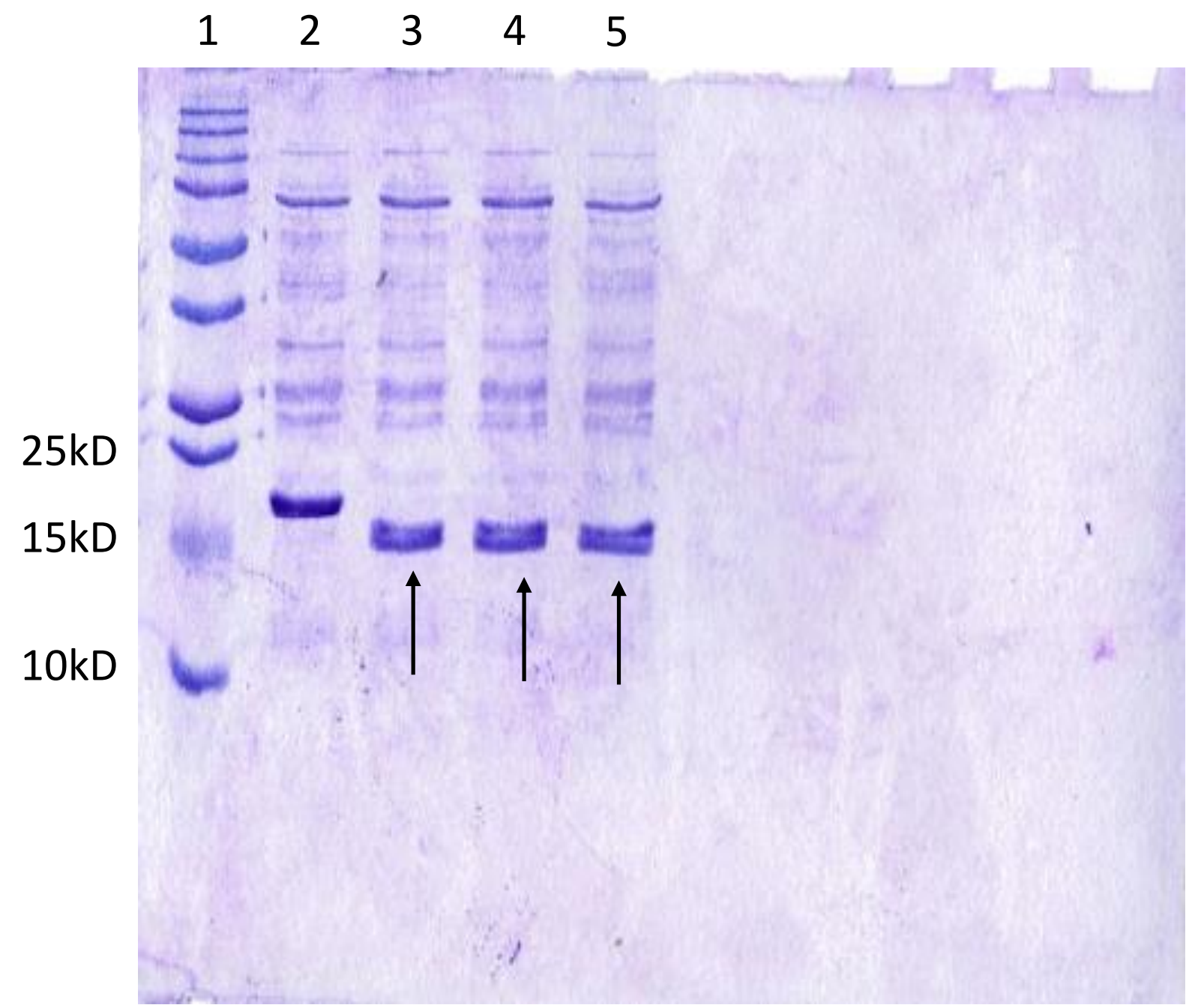

Figure 23. 15\% SDS-PAGE tracking the thrombin digest of His6-LeuRS-I (789-934). The digestion of $\mathrm{C}$-terminal protein with thrombin was initiated by the addition of 2 units of thrombin to the dialyzed C-terminal solution. Lane assignments are as follows: 1. $250 \mathrm{kDa}$ Ladder (Goldbio), 2. undigested His 6 -LeuRS-I (789-934), 3. 2-hour digestion of His6-LeuRS-I (789-934), 4. 4-hour digestion of His6-LeuRS-I (789-934), 5. 16-hour digestion of His6-LeuRS-I (789-934). Cleaved protein exhibits a size of $17.3 \mathrm{kDa}$ and are indicated (arrows) 


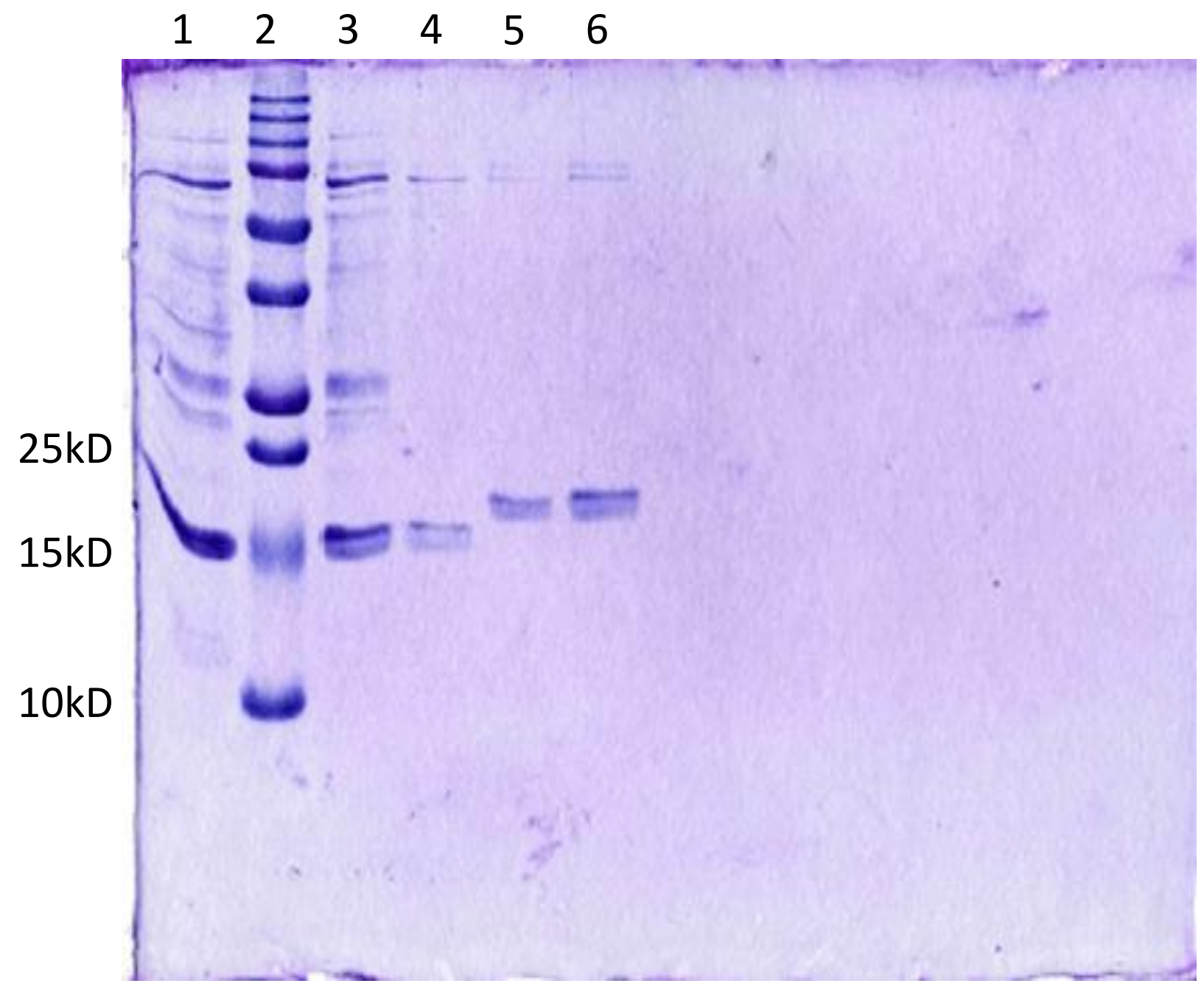

Figure 24. 15\% SDS-PAGE monitoring the His-tag removal from cleaved LeuRS-I (789934). Liberated His-tags were removed via NTA-Ni ${ }^{2+}$ resin. Lane assignments are as follows: 1. digested His 6 -LeuRS-I (789-934) with contaminants, 2. $250 \mathrm{kD}$ protein ladder (GoldBio), 3. digested His 6 -LeuRS-I (789-934) with contaminants (duplicate of lane 1), 4. digested and purified His $_{6}$-LeuRS-I (789-934), 5. undigested and dialyzed His 6 -LeuRS-I(789-934), 6. undigested and dialyzed His6-LeuRS-I (789-934) (duplicate of lane 5). 
Because of the relatively low isoelectric point of LeuRS-I (789-934) $(\sim 5-6)$, anion exchange chromatography utilizing DEAE cellulose resin was used for further purification. The purification was tracked via 15\% SDS-PAGE (Figure 25). After binding under low salt conditions, a step-wise salt gradient was used to elute LeuRS-I (789-934) from the resin. As can be seen (Figure 25, Lanes $3-8$ ), cleaved LeuRS-I (789-934) elutes from the DEAE resin at a salt concentration of $75 \mathrm{mM}$. Interestingly, this ion-exchange chromatography method was effective at not only separating the cleaved protein from the high molecular weight species, but also from the second, upper band within the doublet visualized in Figures 23 and 24 (Figure 25, compare Lanes 6 and 7). The high molecular weight species were eluted at a salt concentration of $150 \mathrm{mM}$ (Figure 25, Lanes 9 and 10). Fractions $1-6$, corresponding to lanes 3 - 8, containing the greater than $98 \%$ pure cleaved LeuRS-I (789-934) were pooled, dialyzed into $10 \mathrm{mM} \mathrm{Na} 2 \mathrm{HPO}_{4}, 2 \mathrm{mM}$ $\mathrm{KH}_{2} \mathrm{PO}_{4}, 137 \mathrm{mM} \mathrm{NaCl}, 2.7 \mathrm{mM} \mathrm{KCl}, 2 \mathrm{mM}$ EDTA, $1 \mathrm{mM}$ DTT, 5\% glycerol, pH 7.5, concentrated to $0.786 \mathrm{mg} / \mathrm{mL}$, and provided to Pierce Custom Antibody Services (Thermo Scientific, $2.5 \mathrm{~mL}$ ) to develop a rabbit polyclonal antibody (2 rabbits: AB1806 and AB1807, 72 day protocol). 


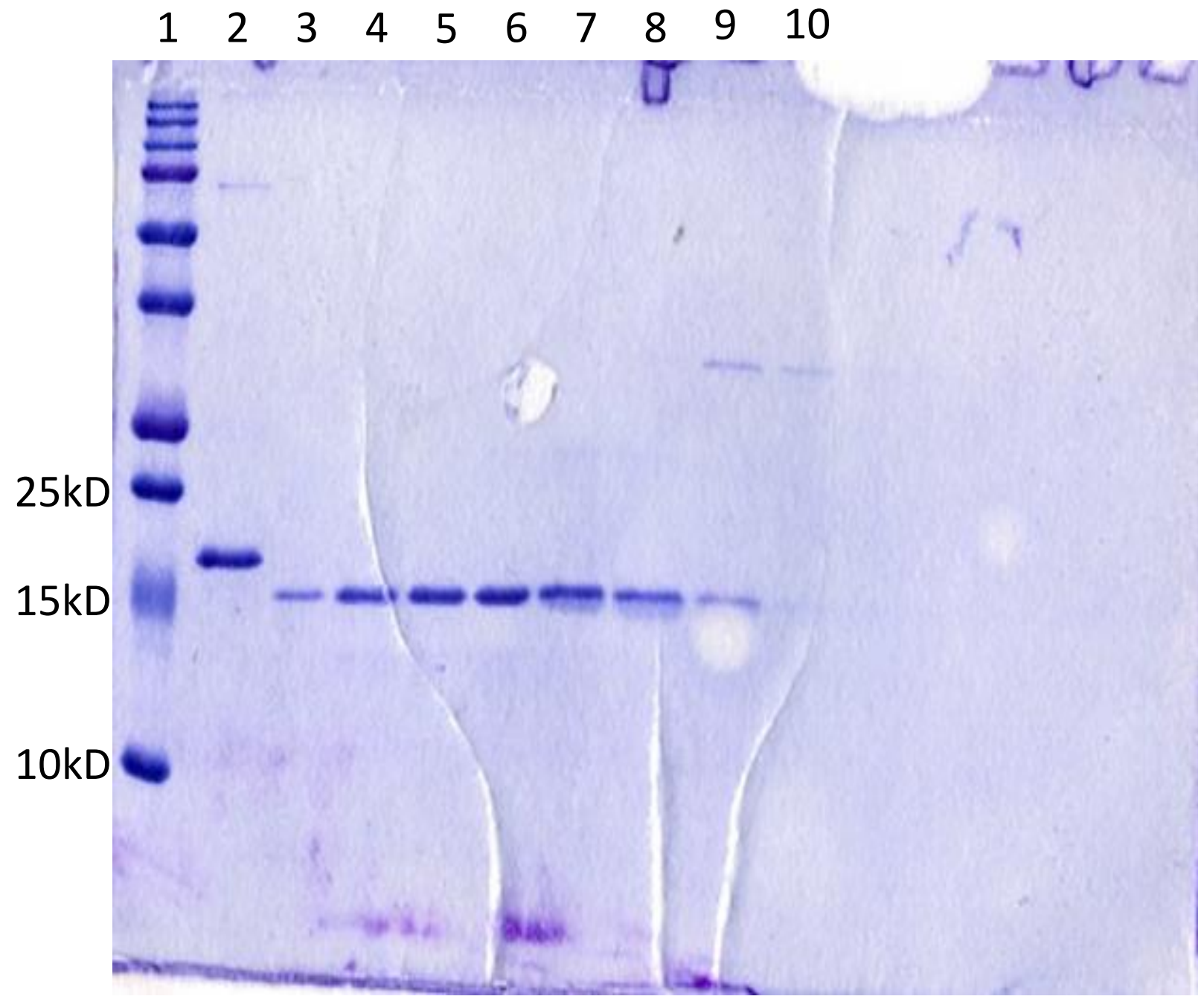

Figure 25. 15\% SDS-PAGE monitoring the anion exchange purification of cleaved LeuRSI (789-934). The removal of a contaminating protein from the $\mathrm{C}$-terminal domain protein was facilitated by anion exchange chromatography using DEAE cellulose resin and increasing concentrations of $\mathrm{NaCl}(75 \mathrm{mM}$ to $150 \mathrm{mM})$. Lane assignments are as follows: 1. 250kD ladder (Goldbio), 2. undigested and dialyzed His 6 -LeuRS-I (789-934), 3 - 8. purified and digested His $6^{-}$ LeuRS-I (789-934), 9 \& 10. contaminant protein. 


\section{HA-I plasmid construction}

As a secondary approach to gauge in vivo expression levels of LeuRS-I, we aimed to generate a strain with wild type growth behavior that encoded a genomic HA-tagged LeuRS-I. This particular design would facilitate experimentation by using a commercially available antibody that can detect the HA epitope. The construction of a HA-tag/LeuRS-I fusion on the chromosome of S. islandicus M.16.4 was carried out utilizing a previously developed pRJW08 suicide vector. ${ }^{[1]}$ To begin, the assemblage of components integral to the formation of this LeuRS-I construct was performed using a Gibson assembly methodology to link a pre-constructed gene fragment (gBlock) constituting a 730 3' segment of the LeuRS-I gene (Tg) fused to the sequence for the HA tag and a 868 bp downstream fragment (D1, IDT), with pRJW08 (Figure 26, plasmid map). ${ }^{[52]}$ Following the assembly of the gBlock fragment into pRJW08 and transformation, resulting colonies from both room temperature and $37^{\circ} \mathrm{C}$ plates were screened to identify clones with the successfully generated recombinant plasmid. Two clones from both temperatures were identified and the colonies from which were miniprepped and screened for the gBlock fragment (Figure 27).

Following a secondary screen of these intermediary plasmids to ensure their integrity through culturing and isolation from E. coli, a second DNA fragment (D2) identical in every way to D1 except its location within pRJW08 was generated via PCR and ligated into pRJW08gBlock $(+)$ (see Figure 26, plasmid map). This second downstream gDNA fragment is required for the proper integration and subsequent removal of plasmid-borne genes to and from S. islandicus (Figure 27). Colony screening of competent cells transformed with this ligated plasmid were screened via PCR and a positive integrant was found (Figure 27, blue block). Cells harboring this D2 positive vector were carried forward and re-screened for the gBlock fragment to ensure that the proper elements were unfettered following ligation and transformation of the D2 fragment 
(Figure 28). Figure 29 details the important elements integrated into the pRJW08 vector in addition to the markers needed to facilitate the introduction of the HA-tag onto the C-terminus of LeuRS-I.

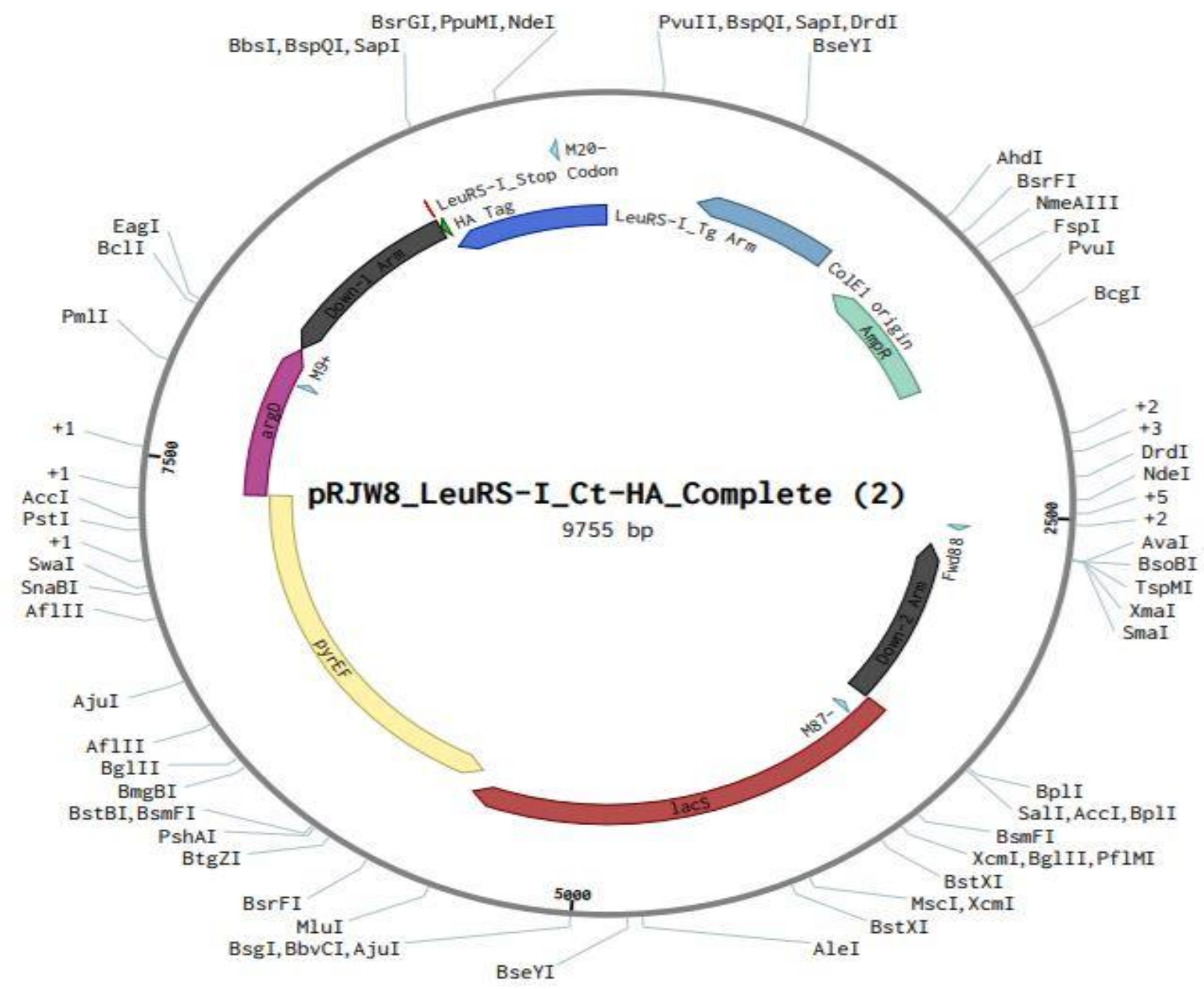

Figure 26. Vector map of the pRJW08-gBlock/D2(+) (HA-I). The HA-I vector map was developed using Benchling [Biology software]. (2020) retrieved from Benchling.com. The vector map notes the important genetic elements required for proper transformation and introduction into $S$. islandicus. The pyrEF (crème) and $\operatorname{arg\mathrm {D}}$ (purple) genes are selectable markers, while lac $\mathrm{S}$ (brick red) allows for Blue/White screening. Other DNA fragments highlighted: Tg arm, dark blue; HA-tag. green, Down-1 arm (D1), black; Down-2 arm (D2), black - opposite D1. These DNA fragments are required for recombination and folding out (or, exclusion) of unnecessary plasmid-borne elements following isolation and counterselection of integrant-positive cells. 


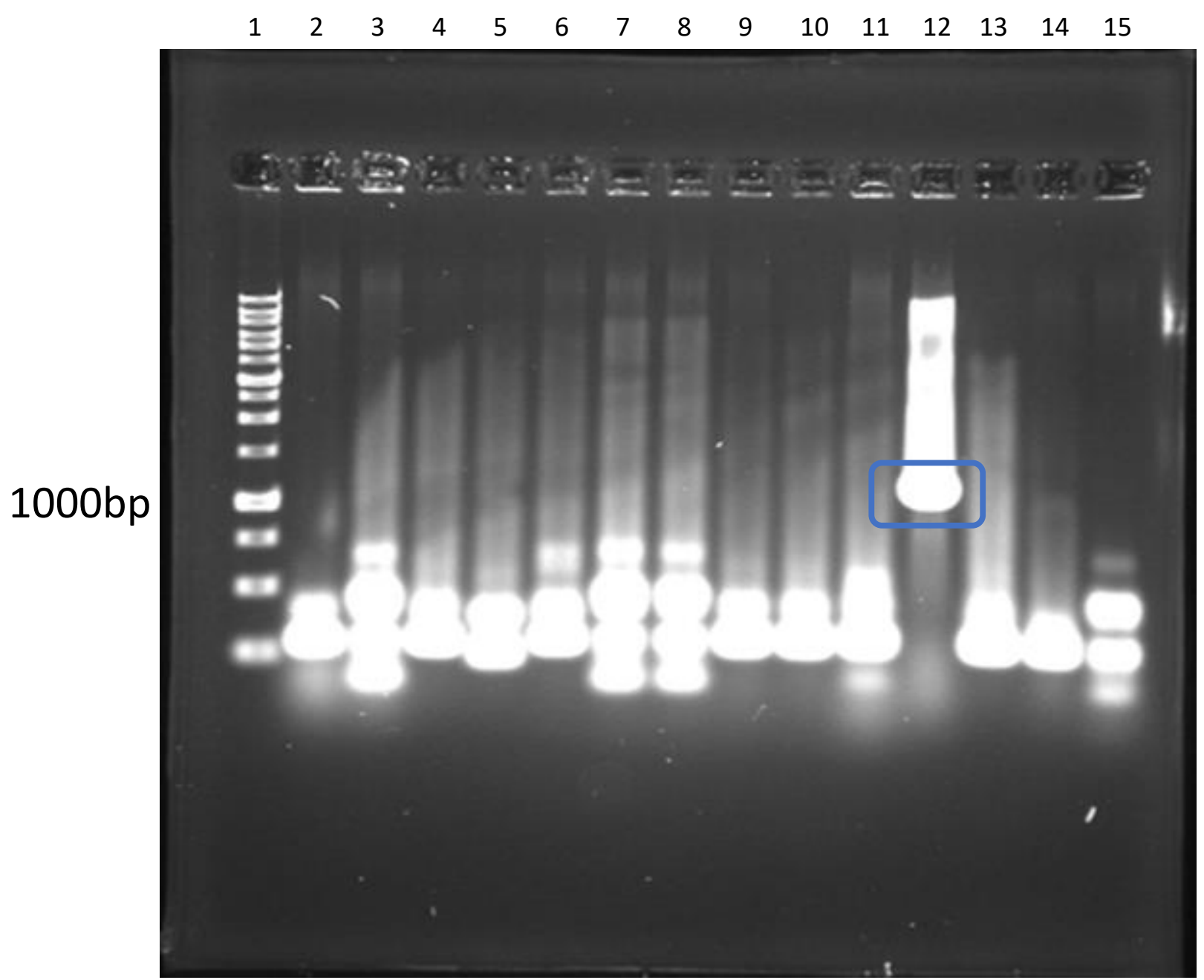

Figure 27. 1.5\% agarose gel depicting the introduction of the D2 fragment into pRJW08gBlock(+). The previously assembled pRJW08-gBlock(+) vector was ligated with a secondary fragment, D2, and was transformed into competent cells. Screening of these colonies for the D2 fragment was performed using primer pair M87 and FOR88. Lane 12 indicates a positive screen of the D2 fragment (circled, blue), 867 bp in size. 


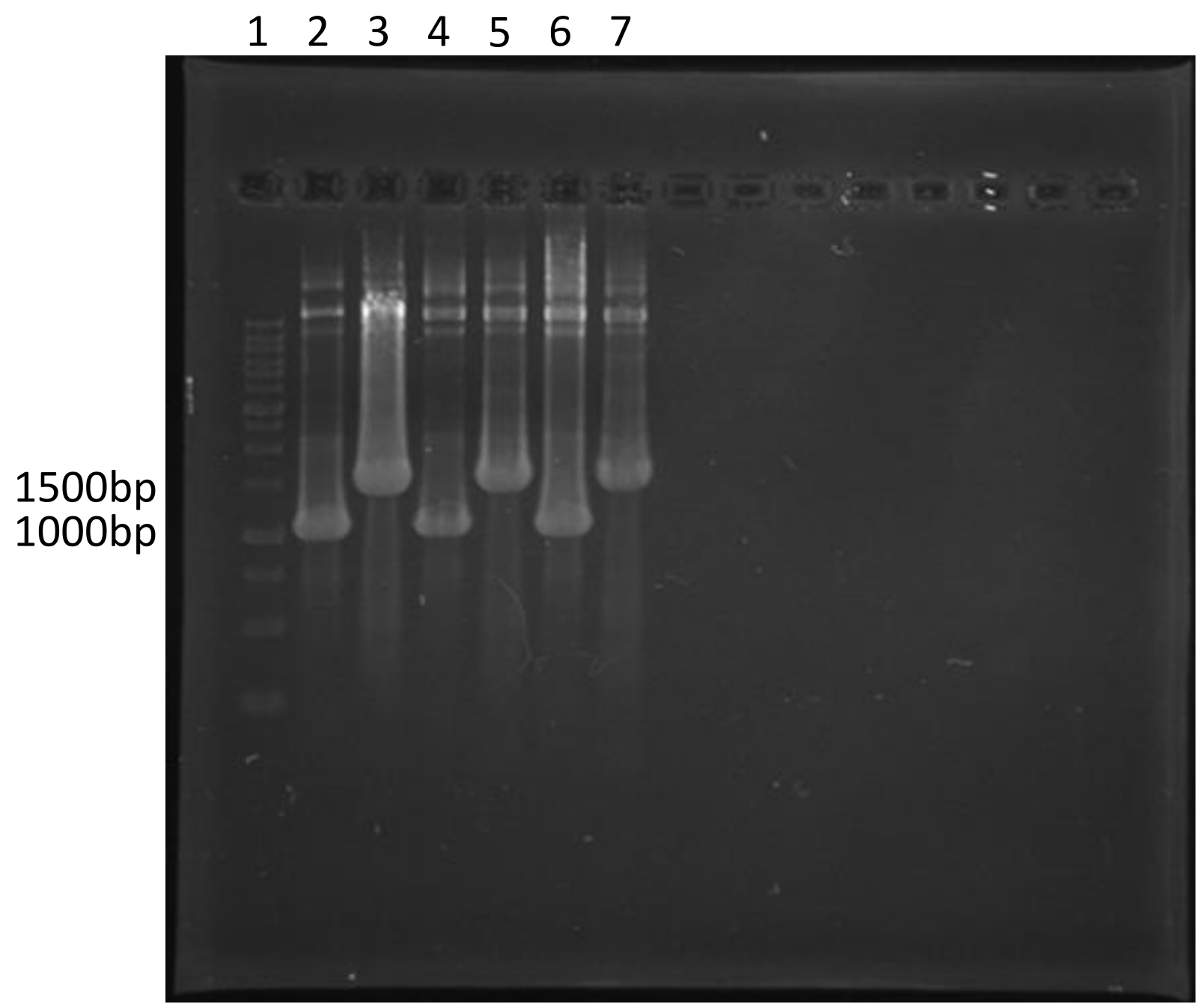

Figure 28. 1.0\% agarose gel depicting the introduction of the D2 and gBlock fragment within discrete minipreps of the positive pRJW08-gBlock/D2(+) vector (HA-I). The positively screened HA-I vector was miniprepped in three distinct elution's and screened for each introduced element (gBlock and D2). Screening of these plasmid preps for the D2 fragment was performed using primer pair M87 and FOR88, as shown in lanes 2, 4, and 6. Lanes 3, 5 and 7 indicate screening of the gBlock fragment with primer pair M9 and REV99. Expected sizes for the D2 and gBlock fragments were $867 \mathrm{bp}$ and $1598 \mathrm{bp}$, respectively 


\section{Transformation of $S$. islandicus wild type with the HA-I vector}

Following the successful construction and sequence verification of the HA-I construct, it was linearized and used to transform our working S. islandicus wild-type strain. Integration of the linearized plasmid into $S$. islandicus ' genome and the subsequent removal of unnecessary plasmidborne components operates under the principle of modified Marker Insertion and unmarked gene Deletion (MID) methodology. ${ }^{[53]}$ Following the insertion of the plasmid at the genomic target locus of LeuRS-I and the upstream nucleoside hydrolase, 5-FOA counterselection invokes the "popping out" of extraneous genetic material ${ }^{[54]}$, namely by random genomic dynamics allowing both the D1 and D2 fragments to overlap and extrude out the selectable marker system first introduced with the plasmid as a circular suicide vector. Various amounts of the linearized vector were used for these experiments, ranging from $0.5 \mu \mathrm{g}$ to $1.5 \mu \mathrm{g}$. Both $1.0 \mu \mathrm{g}$ and $1.5 \mu \mathrm{g}$ of vector yielded a number of colonies ideally representing clones with the vector inserted after the 3 ' end of the gene coding for LeuRS-I (Figure 29). X-gal staining of colonies harvested from both of these plates indicated a strong blue hue showing proper introduction of the linearized HA-I vector into its genomic destination in selected strains $1 \mu \mathrm{g}$ P1 (Pick 1), $1 \mu \mathrm{g}$ P5, $1.5 \mu \mathrm{g}$ P2 and $1.5 \mu \mathrm{g}$ P4 isolates

(Figure 30). X-gal positive strains were colony purified, and these strains maintained blue staining in the presence of this lactose analog (data not shown). Counterselections to remove the unnecessary plasmid-borne components, but maintain the HA C-terminal fusion of LeuRS-I (Figure 31), resulted in the removal of the lacS gene, an ortholog of beta-galactosidase that elicits the metabolism of lactose and, therefore, the indicative presence of blue within the sample. Therefore, counterselected strains did not produce blue pigment upon exposure to X-gal, indicating the removal of these extraneous genes (lac $\mathrm{S}, \operatorname{pyr} \mathrm{EF}, \arg \mathrm{D})$ from the genome of these fusion strains. Following counterselection, the transformants were screened using an HA specific primer to 
elucidate the presence of this genomically located epitope tag (Figure 31). Moreover, clones were further verified using Sanger sequencing at the Core DNA Facilities at UIUC.

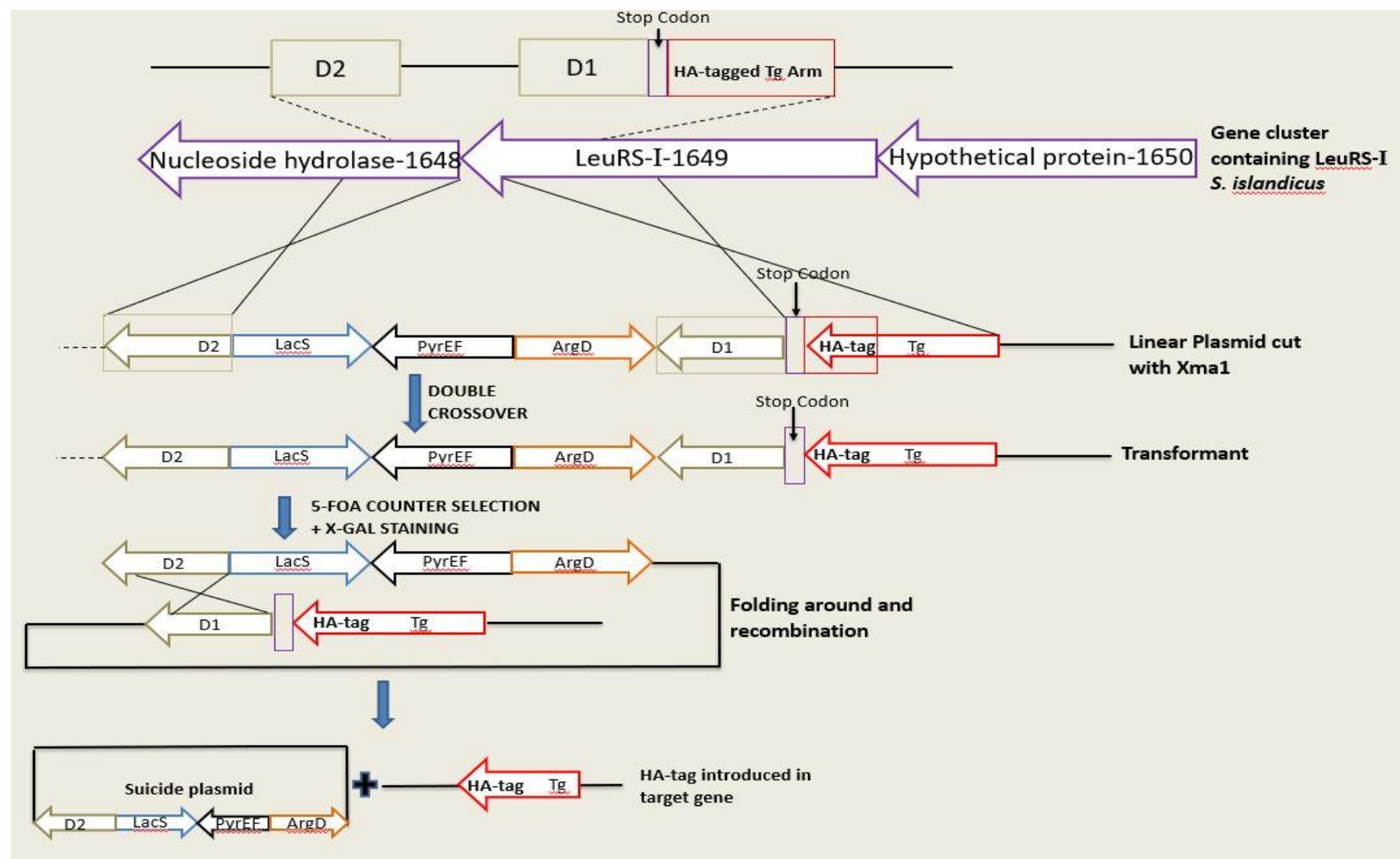

Figure 29. Modified MID recombination scheme for introduction of a C-terminal HA tagged fusion with leuRS-I. Recombination scheme depicting linearized PRJW08 and its insertion into $S$. islandicus' genome as well as the removal of extraneous genetic elements from $S . i$ wild type's genome using a modified MID methodology. Overlapping of the D1 and D2 fragments, which are identical sequences, during 5-FOA counterselection causes the selection cassette comprised of $l a c \mathrm{~S}, p y r \mathrm{EF}$, and $\arg D$ to be extruded, leaving behind a genomically HA tagged variant of LeuRSI. 


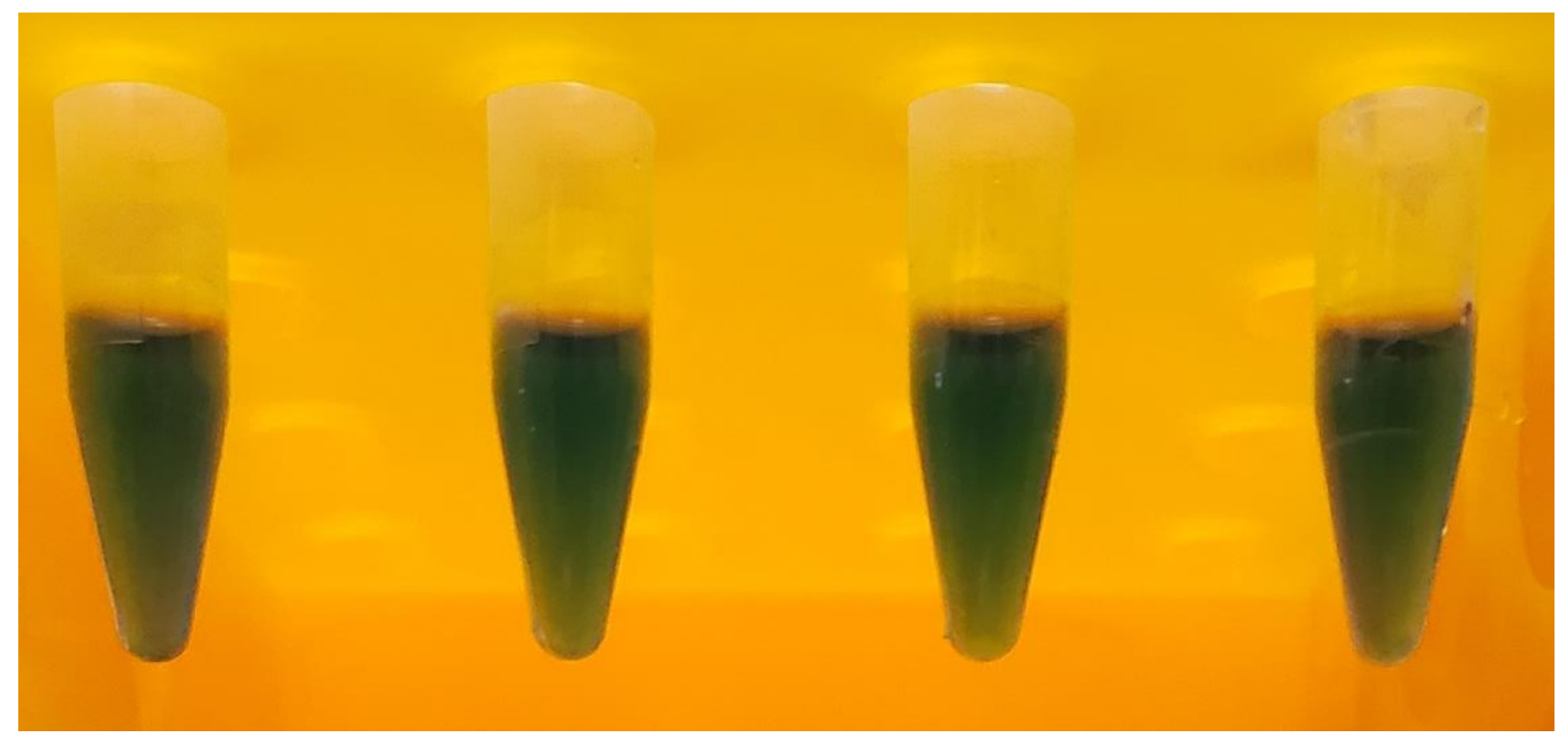

Figure 30. X-gal staining of $\boldsymbol{S}$. islandicus with integrated HA-I. Following the transformation if $S$. islandicus with the HA-I vector, colonies were harvested and grown in DT medium then subsequently stained with X-gal. Microfuge tubes 1 and 2 indicate colonies derived from the $1 \mu \mathrm{g}$ transformation plates, samples $1 \mu \mathrm{g} \mathrm{P} 1$ and $1 \mu \mathrm{g}$ P5, respectively. Microfuge tubes 3 and 4 relate to colonies derived from the $1.5 \mu \mathrm{g}$ transformation plate, samples $1.5 \mu \mathrm{g}$ P2 and $1.5 \mu \mathrm{g}$ P4, respectively. This selection of cultures is not indicative of the total set of transformants that screened positive with X-gal, but are a select few that indicated blue pigment production. 


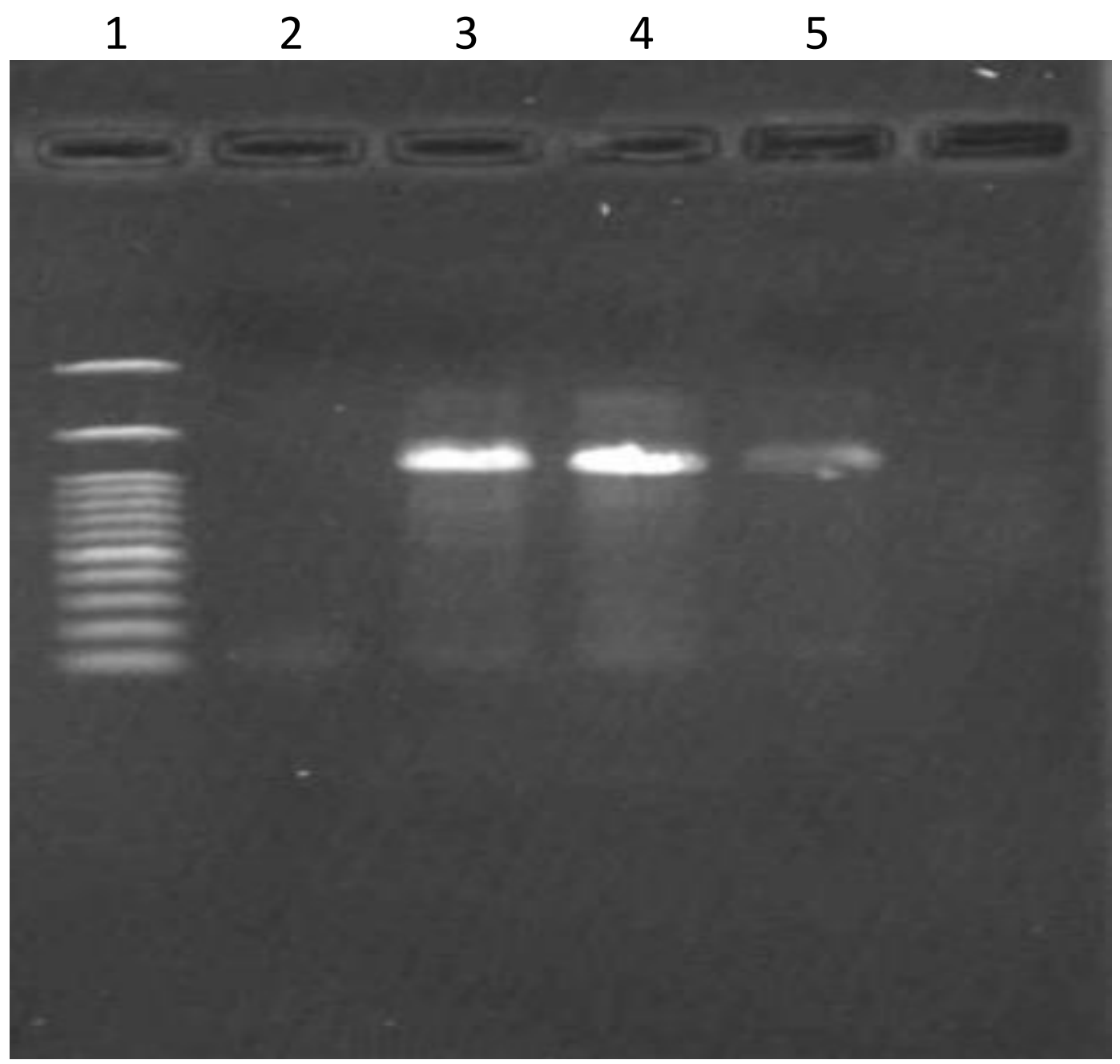

Figure 31. 2\% agarose gel depicting screens of the genomically integrated HA tag in $S$. islandicus M.16.4. Purified and counterselected strains were screened using primer pair LRSI 1724 and M97, the latter being a primer that binds specifically to the HA tag. Lane assignments are as follows: 1. 100bp ladder (Goldbio), 2. Sample $1 \mu \mathrm{g} \mathrm{P1,} \mathrm{3.} \mathrm{Sample} 1 \mu \mathrm{g} \mathrm{P2,} \mathrm{4.} \mathrm{Sample} 1 \mu \mathrm{g}$ P3, 5. Sample $1 \mu \mathrm{g}$ P5. Positive screens indicate a band slightly above the 1000bp band on the ladder. Expected band size: 1042 bp 


\section{Growth curve of the HA-I strain with $S$. islandicus wild type strain}

The ability to utilize the newly generated HA-I strain in expression profiling was predicated on its ability to possess a phenotype close in nature to wild type $S$. islandicus cells. If the strain were to behave in a strange manner as compared to wild type $S$. islandicus, it would render any results obtained from expression profiling as confounded and dubious given the possibility of the HA tag impacting the activity of LeuRS-I or of errant genetic abnormalities incurred during the development of the HA-I strain. The stressful temperature of $60^{\circ} \mathrm{C}$ was employed to exacerbate any phenotypic differences from the HA-I strain in comparison to $S$. islandicus wild type. Growth curve data indicated a strong tracking of optical densities between the triplicates employed within the experiment with little deviation of note (Figure 32). Maximum optical densities achieved were 0.532 and 0.528 for $S$. islandicus wild type and the HA-I strain, respectively, with a death phase concurrent between the two strains on day 8 of the growth curve. 


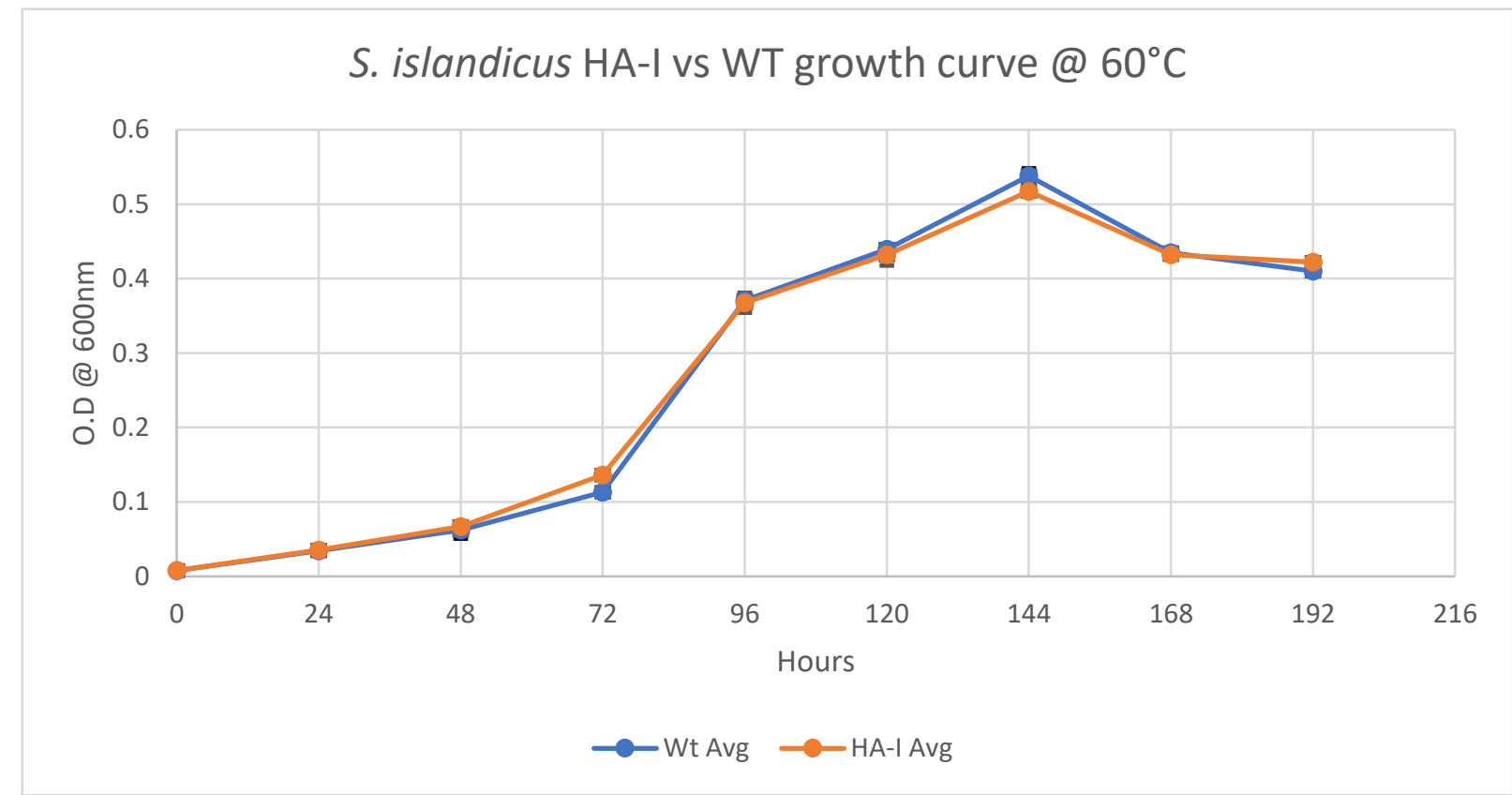

Figure 32. Growth curve data of $S$. islandicus wild type strain and the HA-I strain. O.D $600 \mathrm{~nm}$ values were gathered at the same time of each day in 24 hour increments over the course of 9 days. Data represents triplicate averaging between the two strains. Error bars are present but are nearly indistinguishable at each data point due to the tight tracking of optical density between the two strains 


\section{Expression profiling of the HA-I strain}

In an attempt to better understand the in vivo expression of LeuRS-I within S. islandicus and assess whether such expression could be modulated with the introduction of stressful growth temperatures, an expression profiling experiment was performed using the HA-I strain and FLAGF strain. Following the assessment of the HA-I strain within a growth curve, it was determined that the strain was suitable for expression profiling via western blot analysis. Cultures grown at $75^{\circ} \mathrm{C}$ and $60^{\circ} \mathrm{C}$ were harvested at variable optical densities equating to low (LOD), mid (MOD) and high (HOD) optical densities to assess any possible temporal expression or modulation within given stages of growth under the two conditions. Due to a loss of roughly half of the volume in one of the HA-I flasks at $60^{\circ} \mathrm{C}$, lysis conditions were halved accordingly. Following the lysis and quantitation of protein from each sample, SDS-PAGE was run following the standardization to 1.5 $\mu \mathrm{g}$ total loaded protein within each sample. Western blot analysis indicated the presence of HA-I within all collected samples at both growth temperatures on x-ray film (Figure 33). Densitometry assessment revealed interesting patterns of expression of LeuRS-I in vivo, with a clear indication of importance of the enzyme at low optical densities within both the standard and low temperature growth cultures (Figure 34). A steep decline in the expression of LeuRS-I at $60^{\circ} \mathrm{C}$ as compared to the relatively maintained expression of the protein during MOD at $75^{\circ} \mathrm{C}$ was noted, though the implication of such data is unclear at this moment. The expression of LeuRS-I at HOD appears reduced in both the $60^{\circ} \mathrm{C}$ and $75^{\circ} \mathrm{C}$ samples, which could be an artifact of cells harvested at these high optical densities being at or close to their death phase, or because the expression of LeuRS-I is downregulated at such high optical densities. Densitometric data was generated using FIJI. The FLAG-F strain did not show any signal when probing via western blot, so it has been excluded from any densitometric testing. 


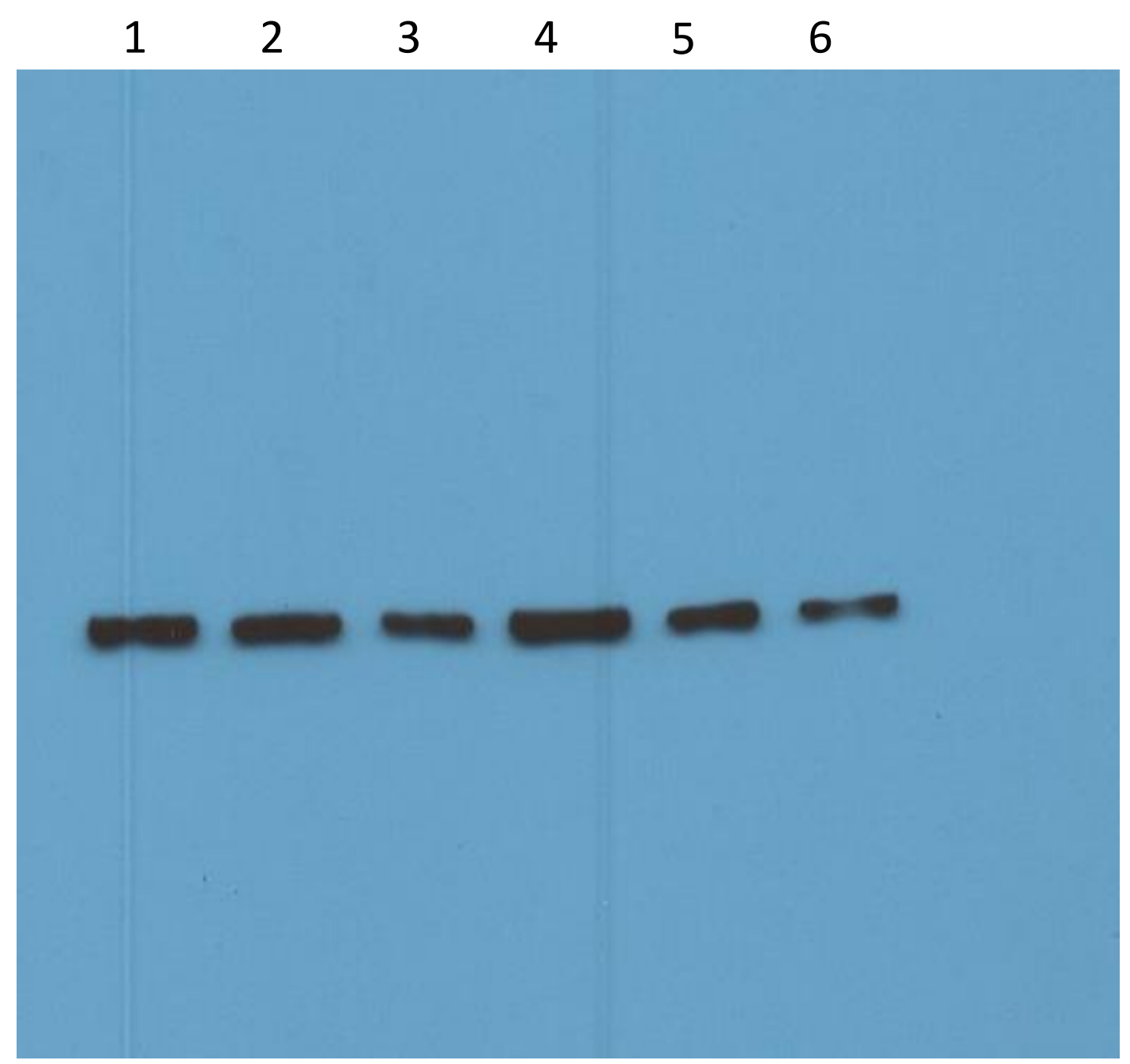

Figure 33. X-ray film depicting the expression of HA-I across differential temperatures and optical densities. Western blot analysis performed on the HA-I strain at the temperatures of $60^{\circ} \mathrm{C}$ and $75^{\circ} \mathrm{C}$ at low (LOD), mid (MOD), and high (HOD) optical densities. The blot was developed using a calculated $1.5 \mu \mathrm{g}$ of total protein within each lane of an $8 \%$ stacking, $10 \%$ resolving SDS-PAGE. Order of samples is as follows: $1.60^{\circ} \mathrm{C} \mathrm{HA}-\mathrm{I}(\mathrm{LOD}), 2.75^{\circ} \mathrm{C} \mathrm{HA}-\mathrm{I}$ (LOD), 3. $60^{\circ} \mathrm{C}$ HA-I (MOD), 4. $75^{\circ} \mathrm{C}$ HA-I (MOD), 5. $60^{\circ} \mathrm{C}$ HA-I (HOD), 6. $75^{\circ} \mathrm{C}$ HA-I (HOD) 


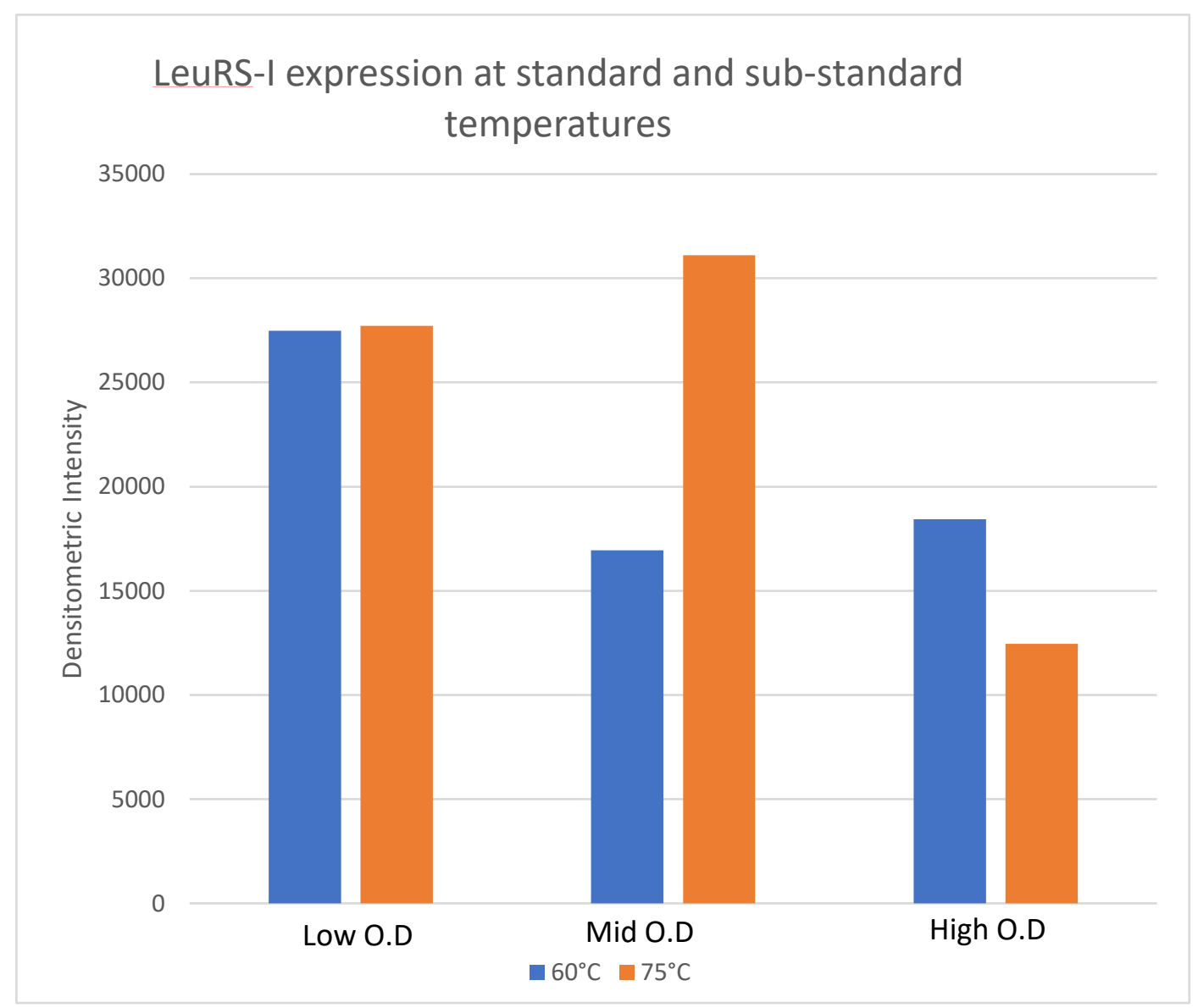

Figure 34. Densitometric intensity counts of LeuRS-I expression at $75^{\circ} \mathrm{C}$ and $60^{\circ} \mathrm{C}$. FIJI was utilized to develop standardized densitometric counts of in vivo LeuRS-I expression via histogram area analysis. Background subtraction was performed using blank X-ray film and subtracting the given counts from all sample densitometric readings. Expression is portrayed as arbitrary units of densitometric intensity. 


\section{CHAPTER IV: CONCLUSION}

As presented, a variety of angles have been probed in an attempt understand the functional relevance of LeuRS-I in Sulfolobus islandicus. While this question is left unanswered in full, a few key insights have been made that act as steppingstones towards potentially fruitful experimentation that could ultimately answer this unknown. Given LeuRS-I's lack of activity within aminoacylation reactions, it is indeed a startling discovery that not only does LeuRS-I bind in vitro transcribed tRNA ${ }^{\text {Leu }}-\mathrm{UAG}$, but it does so at roughly the same binding affinity as LeuRS-F. This brings into light a possibility in which LeuRS-I either requires a specifically modified version of tRNA in which to impose its aminoacylating properties upon, or it requires accessory proteins to elicit aminoacylation. With the use of the custom developed polyclonal antibody, purifying LeuRS-I and potential accessory proteins out of wild type S. islandicus is possible, making the latter possibility testable.

And yet, preliminary aminoacylation reactions with LeuRS-I utilizing endogenously extracted and post-transcriptionally modified tRNA's appear negative for aminoacylation activity, bringing into question its role as a canonical synthetase. The potential exists in which LeuRS-I has maintained its ability to bind tRNA due to its core aesthetic of being an aminoacyltRNA synthetase, but has been evolved over an ancient timescale to perform an exotic secondary function such as charging amino acids on to proteins to modify their chemical properties or generating small molecules of utility to $S$. islandicus. Such possibilities could be tested using modified aminoacylation reactions that track the attachment of amino acids onto alternative proteins or by utilizing mass spectrometry to identify substitutions of amino acids on these proteins. 
Phylogenetic analysis of the LeuRS enzymes within the Sulfolobaceae has provided a strong argument for this paralog's maintenance within every member of the family, including strains separated by geographical barriers of thousands of miles. Due to the omnipresence of LeuRS-I within these organisms, it is not difficult to imagine that this paralog exhibits a strong functional relevance to the species, though it is ultimately unknown what this importance is. Analysis of LeuRS-I's expression in response to sub-standard growth temperatures was a good starting point for determining if this paralog's expression can be modulated by abiotic stress, but a host of other experiments can be utilized in an attempt to exacerbate this modulation including differential environmental $\mathrm{pH}$ 's and oxidative stress experiments. Indeed, further use of the molecular tools generated within this thesis could help untangle the mystery behind the activity of this unique paralog within S. islandicus. 


\section{REFERENCES}

1. Freist, W. (1989) Mechanisms of aminoacyl-tRNA synthetases: a critical consideration of recent results. Biochemistry. 28, 6787-6795

2. Schimmel, P. R., and Söll, D. (1979) Aminoacyl-tRNA Synthetases: General Features and Recognition of Transfer RNAs. Annu. Rev. Biochem. 48, 601-648

3. Blanchet, S., Cornu, D., Hatin, I., Grosjean, H., Bertin, P., and Namy, O. (2018) Deciphering the reading of the genetic code by near-cognate tRNA. Proc. Natl. Acad. Sci. 115, 3018 LP - 3023

4. Saito, K., Kobayashi, K., Wada, M., Kikuno, I., Takusagawa, A., Mochizuki, M., Uchiumi, T., Ishitani, R., Nureki, O., and Ito, K. (2010) Omnipotent role of archaeal elongation factor 1 alpha $(\mathrm{EF} 1 \alpha)$ in translational elongation and termination, and quality control of protein synthesis. Proc. Natl. Acad. Sci. 107, 19242 LP - 19247

5. Ribas de Pouplana, L., and Schimmel, P. (2001) Two Classes of tRNA Synthetases Suggested by Sterically Compatible Dockings on tRNA Acceptor Stem. Cell. 104, 191-193

6. Brown, J. R., and Doolittle, W. F. (1995) Root of the universal tree of life based on ancient aminoacyl-tRNA synthetase gene duplications. Proc. Natl. Acad. Sci. 92, 2441 LP - 2445

7. Iwabe, N., Kuma, K., Hasegawa, M., Osawa, S., and Miyata, T. (1989) Evolutionary relationship of archaebacteria, eubacteria, and eukaryotes inferred from phylogenetic trees of duplicated genes. Proc. Natl. Acad. Sci. 86, 9355 LP - 9359 
8. Brock, T. D., Brock, K. M., Belly, R. T., and Weiss, R. L. (1972) Sulfolobus: a new genus of sulfur-oxidizing bacteria living at low pH and high temperature. Arch. Mikrobiol. 84, 5468

9. Guo, L., Brügger, K., Liu, C., Shah, S. A., Zheng, H., Zhu, Y., Wang, S., Lillestøl, R. K., Chen, L., Frank, J., Prangishvili, D., Paulin, L., She, Q., Huang, L., and Garrett, R. A. (2011) Genome analyses of Icelandic strains of Sulfolobus islandicus, model organisms for genetic and virus-host interaction studies. J. Bacteriol. 193, 1672-1680

10. Chen, L., Brügger, K., Skovgaard, M., Redder, P., She, Q., Torarinsson, E., Greve, B., Awayez, M., Zibat, A., Klenk, H.-P., and Garrett, R. A. (2005) The Genome of Sulfolobus acidocaldarius, a Model Organism of the Crenarchaeota; J. Bacteriol. 187, 4992 LP - 4999

11. Zhang, C., Cooper, T. E., Krause, D. J., and Whitaker, R. J. (2013) Augmenting the genetic toolbox for Sulfolobus islandicus with a stringent positive selectable marker for agmatine prototrophy. Appl. Environ. Microbiol. 79, 5539-5549

12. Guo, L., Brügger, K., Liu, C., Shah, S. A., Zheng, H., Zhu, Y., Wang, S., Lillestøl, R. K., Chen, L., Frank, J., Prangishvili, D., Paulin, L., She, Q., Huang, L., and Garrett, R. A. (2011) Genome analyses of Icelandic strains of Sulfolobus islandicus, model organisms for genetic and virus-host interaction studies. J. Bacteriol. 193, 1672-1680

13. Reno, M. L., Held, N. L., Fields, C. J., Burke, P. V, and Whitaker, R. J. (2009) Biogeography of the Sulfolobus islandicus pan-genome. Proc. Natl. Acad. Sci. U. S. A. 106, $8605-8610$ 
14. Feng, X., Liu, X., Xu, R., Zhao, R., Feng, W., Liao, J., Han, W., and She, Q. (2020) A Unique B-Family DNA Polymerase Facilitating Error-Prone DNA Damage Tolerance in Crenarchaeota. Front. Microbiol.. 11, 1585

15. Weitzel, C. S., Li, L., Zhang, C., Eilts, K. K., Bretz, N. M., Gatten, A. L., Whitaker, R. J., and Martinis, S. A. (2020) Duplication of leucyl-tRNA synthetase in an archaeal extremophile may play a role in adaptation to variable environmental conditions. J. Biol. Chem. 10.1074/jbc.RA118.006481

16. Boniecki, M. T., Vu, M. T., Betha, A. K., and Martinis, S. A. (2008) CP1-dependent partitioning of pretransfer and posttransfer editing in leucyl-tRNA synthetase. Proc. Natl. Acad. Sci. 105, 19223 LP - 19228

17. Martinis, S. A., and Boniecki, M. T. (2010) The balance between pre- and post-transfer editing in tRNA synthetases. FEBS Lett. 584, 455-459

18. Moras, D. (2010) Proofreading in translation: Dynamics of the double-sieve model. Proc. Natl. Acad. Sci. 107, 21949 LP - 21950

19. Betha, A. K., Williams, A. M., and Martinis, S. A. (2007) Isolated CP1 Domain of Escherichia coli Leucyl-tRNA Synthetase Is Dependent on Flanking Hinge Motifs for Amino Acid Editing Activity. Biochemistry. 46, 6258-6267

20. Ribas de Pouplana, L., Santos, M. A. S., Zhu, J.-H., Farabaugh, P. J., and Javid, B. (2014) Protein mistranslation: friend or foe? Trends Biochem. Sci. 39, 355-362 
21. Li, L., Boniecki, M. T., Jaffe, J. D., Imai, B. S., Yau, P. M., Luthey-Schulten, Z. A., and Martinis, S. A. (2011) Naturally occurring aminoacyl-tRNA synthetases editing-domain mutations that cause mistranslation in Mycoplasma parasites. Proc. Natl. Acad. Sci. 108, 9378 LP - 9383

22. Netzer, N., Goodenbour, J. M., David, A., Dittmar, K. A., Jones, R. B., Schneider, J. R., Boone, D., Eves, E. M., Rosner, M. R., Gibbs, J. S., Embry, A., Dolan, B., Das, S., Hickman, H. D., Berglund, P., Bennink, J. R., Yewdell, J. W., and Pan, T. (2009) Innate immune and chemically triggered oxidative stress modifies translational fidelity. Nature. 462, 522-526

23. Mohler, K., and Ibba, M. (2017) Translational fidelity and mistranslation in the cellular response to stress. Nat. Microbiol. 2, 17117

24. Schwartz, M. H., and Pan, T. (2016) Temperature dependent mistranslation in a hyperthermophile adapts proteins to lower temperatures. Nucleic Acids Res. 44, 294-303

25. Samhita, L., Raval, P. K., and Agashe, D. (2020) Global mistranslation increases cell survival under stress in Escherichia coli. PLoS Genet. 16, e1008654-e1008654

26. RajBhandary, U. L. (1997) Once there were twenty. Proc. Natl. Acad. Sci. 94, 11761 LP 11763 
27. Rubio, M. A., Napolitano, M., Ochoa de Alda, J. A. G., Santamaria-Gomez, J., Patterson, C. J., Foster, A. W., Bru-Martinez, R., Robinson, N. J., and Luque, I. (2015) Transoligomerization of duplicated aminoacyl-tRNA synthetases maintains genetic code fidelity under stress. Nucleic Acids Res. 43, 9905-9917

28. Chopra, S., Palencia, A., Virus, C., Schulwitz, S., Temple, B. R., Cusack, S., and Reader, J. (2016) Structural characterization of antibiotic self-immunity tRNA synthetase in plant tumour biocontrol agent. Nat. Commun. 7, 12928

29. Andam, C. P., Harlow, T. J., Papke, R. T., and Gogarten, J. P. (2012) Ancient origin of the divergent forms of leucyl-tRNA synthetases in the Halobacteriales. BMC Evol. Biol. 12, 85

30. Woese, C. R., Olsen, G. J., Ibba, M., and Söll, D. (2000) Aminoacyl-tRNA synthetases, the genetic code, and the evolutionary process. Microbiol. Mol. Biol. Rev. 64, 202-236

31. Guo, M., Yang, X.-L., and Schimmel, P. (2010) New functions of aminoacyl-tRNA synthetases beyond translation. Nat. Rev. Mol. Cell Biol. 11, 668-674

32. Ahel, I., Korencic, D., Ibba, M., and Söll, D. (2003) Trans-editing of mischarged tRNAs. Proc. Natl. Acad. Sci. 100, 15422 LP - 15427

33. Hassani, M., Pincus, D. H., Bennett, G. N., and Hirshfield, I. N. (1992) Temperaturedependent induction of an acid-inducible stimulon of Escherichia coli in broth. Appl. Environ. Microbiol. 58, 2704-2707

34. He, M., Wilde, A., and Kaderbhai, M. A. (1990) A simple single-step procedure for smallscale preparation of Escherichia coli plasmids. Nucleic Acids Res. 18, 1660 
35. Aranda, P. S., LaJoie, D. M., and Jorcyk, C. L. (2012) Bleach gel: a simple agarose gel for analyzing RNA quality. Electrophoresis. 33, 366-369

36. Sidorova, N. Y., Hung, S., and Rau, D. C. (2010) Stabilizing labile DNA-protein complexes in polyacrylamide gels. Electrophoresis. 31, 648-653

37. Seo, M., Lei, L., and Egli, M. (2019) Label-Free Electrophoretic Mobility Shift Assay (EMSA) for Measuring Dissociation Constants of Protein-RNA Complexes. Curr. Protoc. nucleic acid Chem. 76, e70-e70

38. Kumar, S., Stecher, G., Li, M., Knyaz, C., and Tamura, K. (2018) MEGA X: Molecular Evolutionary Genetics Analysis across Computing Platforms. Mol. Biol. Evol. 35, 15471549

39. Consortium, T. U. (2018) UniProt: a worldwide hub of protein knowledge. Nucleic Acids Res. 47, D506-D515

40. Database resources of the National Center for Biotechnology Information. (2018) Nucleic Acids Res. 46, D8-D13

41. Kanehisa, M., Sato, Y., Kawashima, M., Furumichi, M., and Tanabe, M. (2015) KEGG as a reference resource for gene and protein annotation. Nucleic Acids Res. 44, D457-D462

42. Rosset, S. (2007) Efficient inference on known phylogenetic trees using Poisson regression. Bioinformatics. 23, e142-e147 
43. Studier, F. W., Rosenberg, A. H., Dunn, J. J., and Dubendorff, J. W. (1990) Use of T7 RNA polymerase to direct expression of cloned genes. Methods Enzymol. 185, 60-89

44. Bradford, M. M. (1976) A rapid and sensitive method for the quantitation of microgram quantities of protein utilizing the principle of protein-dye binding. Anal. Biochem. 72, 248254

45. Rabilloud, T. (2018) Optimization of the cydex blue assay: A one-step colorimetric protein assay using cyclodextrins and compatible with detergents and reducers. PLoS One. 13, e0195755

46. Schindelin, J., Arganda-Carreras, I., Frise, E., Kaynig, V., Longair, M., Pietzsch, T., Preibisch, S., Rueden, C., Saalfeld, S., Schmid, B., Tinevez, J.-Y., White, D. J., Hartenstein, V., Eliceiri, K., Tomancak, P., and Cardona, A. (2012) Fiji: an open-source platform for biological-image analysis. Nat. Methods. 9, 676-682

47. Sambrook, J. (2001) Molecular cloning: a laboratory manual / Joseph Sambrook, David W. Russell (Russell 1957-, D. W. (David W., and Laboratory, C. S. H. eds), Cold Spring Harbor Laboratory, Cold Spring Harbor, N.Y

48. McCloskey, J. A., Graham, D. E., Zhou, S., Crain, P. F., Ibba, M., Konisky, J., Söll, D., and Olsen, G. J. (2001) Post-transcriptional modification in archaeal tRNAs: identities and phylogenetic relations of nucleotides from mesophilic and hyperthermophilic Methanococcales. Nucleic Acids Res. 29, 4699-4706 
49. Hellman, L. M., and Fried, M. G. (2007) Electrophoretic mobility shift assay (EMSA) for detecting protein-nucleic acid interactions. Nat. Protoc. 2, 1849-1861

50. Duchon, A. A., St Gelais, C., Titkemeier, N., Hatterschide, J., Wu, L., and Musier-Forsyth, K. (2017) HIV-1 Exploits a Dynamic Multi-aminoacyl-tRNA Synthetase Complex To Enhance Viral Replication. J. Virol. 91, e01240-17

51. Mueller, A. M., Breitsprecher, D., Duhr, S., Baaske, P., Schubert, T., and Längst, G. (2017) MicroScale Thermophoresis: A Rapid and Precise Method to Quantify ProteinNucleic Acid Interactions in Solution. Methods Mol. Biol. 1654, 151-164

52. Gibson, D. G., Young, L., Chuang, R.-Y., Venter, J. C., Hutchison, C. A., and Smith, H. O. (2009) Enzymatic assembly of DNA molecules up to several hundred kilobases. Nat. Methods. 6, 343-345

53. Zhang, C., Guo, L., Deng, L., Wu, Y., Liang, Y., Huang, L., and She, Q. (2010) Revealing the essentiality of multiple archaeal pcna genes using a mutant propagation assay based on an improved knockout method. Microbiology. 156, 3386-3397

54. Widlund, P. O., and Davis, T. N. (2005) A high-efficiency method to replace essential genes with mutant alleles in yeast. Yeast. 22, 769-774 
APPENDIX A: TABLES

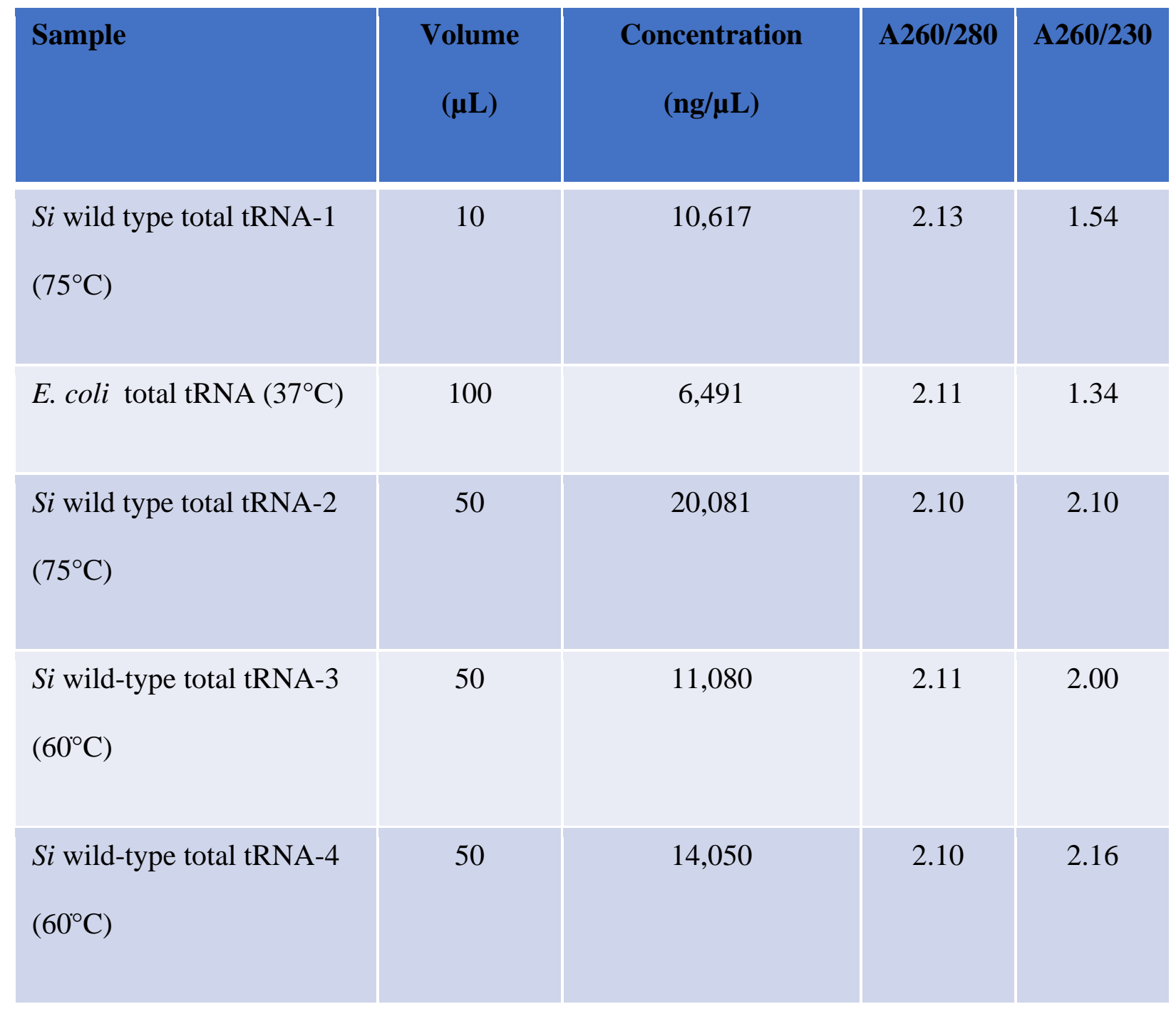

Table A-1: Concentrations of total tRNA preps, unprocessed samples. Total tRNA preps from extracted S. islandicus or E. coli cells, unprocessed. 


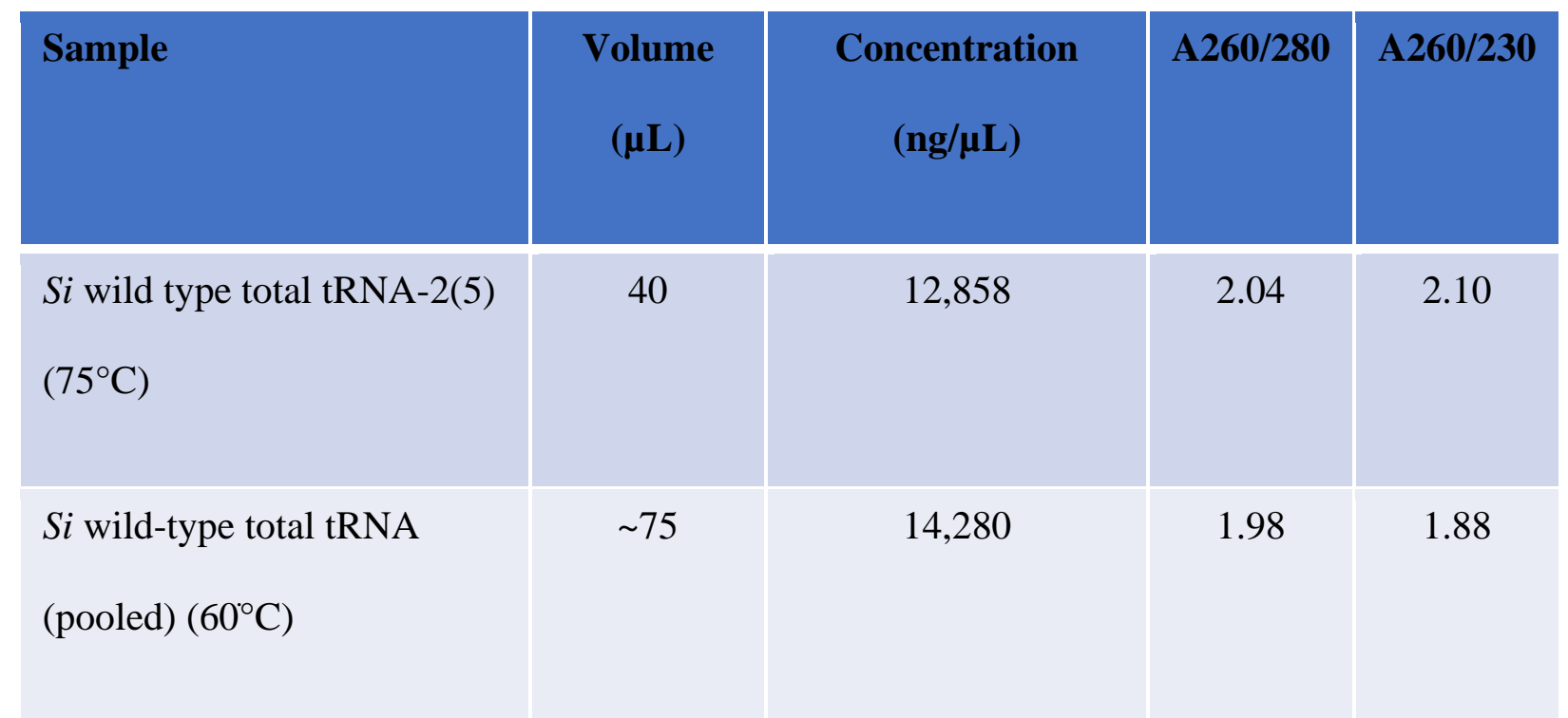

Tables A-2: Concentrations of total tRNA preps (purified). Concentrations and quality assessments of processed and cleaned total tRNA pools from contaminating nucleic acids. 


\begin{tabular}{|l|l|}
\hline Primer name & Application \\
\hline M9 & pRJW08+gBlock \\
\hline Rev99 & pRJW08+gBlock \\
\hline M57 & Downstream 2 (D2) fragment amplification \\
\hline M58 & Downstream 2 (D2) fragment amplification \\
\hline M97 & HA-tag screening, LeuRS-I \\
\hline LRSI 1724 & HA-tag screening, LeuRS-I \\
\hline
\end{tabular}

Table A-3: Primers used in this study. All primers that were used in screening or generation of

plasmids are listed. 